\title{
AVALIAÇÃO DE ALGUNS DELINEAMENTOS COMPOSTOS PEQUENOS PARA EXPERIMENTAÇÃO AGRONÔMICA
}

\author{
GENER TADEU PEREIRA \\ Estatistico
}

Orientador: Prof. Dr. Dilermando Perecin

\begin{abstract}
Tese apresentada à Escola Superior de Agricultura "Luiz de Queiroz", da Universidade de São Paulo, para obtenção do título de Doutor em Agronomia, Área de Concentração: Estatística e Experimentação Agronômica.
\end{abstract}

P I R A C.I C A B A

Estado de são Paulo - Brasil

Junho - 1995 


\section{CATALOGAÇÃO NA PUBLICAÇÃO \\ DIVISÃO DE BIBLIOTECA E DOCUMENTAÇÃO \\ CAMPUS "LUIZ DE QUEIROZ"}

Pereira, Gener Tadeu

Avaliação de alguns delineamentos compostos pequenos para experimentação agronômica. Piracicaba, 1995.

$129 \mathrm{p}$.

Tese - ESALQ

Bibliografia.

1. Delineamento de experimento 2. Experimentação agrícola I. Escola Superior de Agricultura Luiz de Queiroz, Piracicaba

CDD 519.5

630.72 


\section{AVALIAÇÃO DE ALGUNS DELINEAMENTOS COMPOSTOS PEQUENOS PARA EXPERIMENTAÇÃO AGRONÔMICA}

GENER TADEU PEREIRA

Aprovada em: 31.08 .95

Comissão Examinadora:

Prof. Dr. Décio Barbin

ESALQ/USP

Prof. Dra. Maria Cristina Stolf Nogueira ESALQ/USP

Prof. Dr. Dilermando Perecin

FCAV/UNESP

Prof. Dr. Adhemar Sanches

FCAV/UNESP

Dr. Toshio Igue

IAC

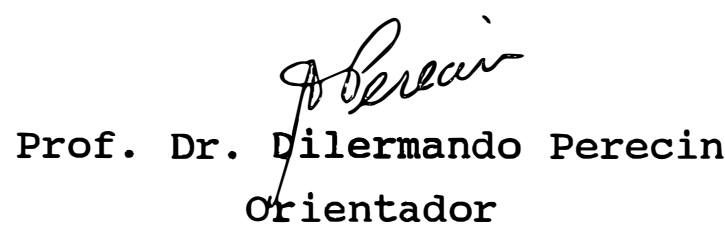


Aos meus pais

MIGUEL PEREIRA e

ADÉLIA PAIVA PEREIRA

DEDICO

À REGINA, minha esposa e aos nossos filhos: LUCAS e ALÁDIA OFEREÇO 


\section{AGRADECIMENTOS}

Ao Prof. Dr. Dilermando Perecin, pela orientação, ensinamentos, constante estímulo e confiança.

Ao Prof. Dr. Adhemar Sanches, pelas proveitosas discussões e sugestões que muito contribuíram para a realização deste trabalho.

Ao Prof. Dr. João Ademir de Oliveira, pelas valiosas sugestões, com vistas ao aprimoramento deste trabalho.

Aos Professores e funcionários do Departamento de Ciências Exatas da Faculdade de Ciências Agrárias e Veterinárias - UNESP, Campus de Jaboticabal, pela oportunidade de aperfeiçoamento.

Aos Professores do Departamento de Matemática e Estatística da ESALQ/USP, pelos ensinamentos.

Aos colegas de curso: César, Eufrásio, Joel, Rosana, Rui, Samuel, Sônia e Carlos Tadeu, pelo alegre convívio.

À Cristiane Trizolio Pascon, pelo dedicado trabalho de digitação deste trabalho.

À Coordenadoria de Aperfeiçoamento de Pessoal de Nível Superior (CAPES/PICD), pela bolsa de estudo concedida.

A todos que, de uma forma ou de outra, contribuíram para a realização deste trabalho. 
I N D I C E

Página

RESUMO vii

SUMMARY $x$

1. INTRODUÇÃa 1

2. REVISĀO DA LITERATURA. 6

3. METODOLOGIA ................................ 15

3.1. o modelo matemático do sistema.............. 15

3.2. o delineamento de tratamentos............... 17

3.3. A notação matricial do modelo................ 18

3.4. A estimação ........................... 18

3.5. Análise da superfície de resposta ajustada ..... 19 3.5.1 Redução à forma canônica.............. 21

3.6. Algumas propriedades e critérios de escolha de delineamentos ....................... 23

3.6.1. Delineamentos contínuos e exatos....... 25 3.6.2. Aspectos teóricos dos critérios ótimos ... 28 3.6.3. Critério da variância da prediçãa ....... 41 3.6.4. Comparação de delineamentos ........... 42 3.6.5. Rotacionalidade................... 43 
3.6.5.1. Medindo a rotacionalidade...... 45

3.7. Delineamentos ................... 48

3.7.1. O Delineamento fatorial2 ${ }^{k} \ldots \ldots \ldots \ldots \ldots 49$

3.7.2. Blocos e delineamentos fatoriais

fracionários $2^{k} \ldots \ldots \ldots \ldots \ldots \ldots \ldots$

3.7.3.Delineamentos fatoriais fracionários $2^{k-m} . .51$

3.7.4. Delineamento composto central........ 52

3.7.5. Delineamentos compostos pequenos ....... 58

3.7.5.1. Delineamentos compostos de

Hartley...................... 58

3.7.5.2. Delineamentos compostos de

Westlake.....................61

3.7.5.3. Delineamentos compostos de

Draper-Lin...................61

3.9.4.4. Os delineamentos compostos de

Lucas.......................63

3.8. Capacidade de reprodução do ponto ótimo e número médio de inversões ............... 64

3.8.1. o método utilizado............6 64

3.8.2 Obtenção de modelos de segunda

ordem com 4 a 7 fatores.............66 66

4. RESUltados E DiscUSSÃo ...................... 73

4.1. Resultados dos critérios ótimos ...........73

4.2. Porcentagem de reprodução do ponto crítico (\%Rep)

e número médio de inversões (NMinv) $\ldots \ldots \ldots \ldots . .83$ 
4.3. A medida de rotacionalidade $\left(Q^{*}\right)$ e os valores

da distância axial ( $\propto$ ) para a obtenção de

arranjos em blocos ortogonais ............ 113

5. CONCLUSÕES ............................ 119

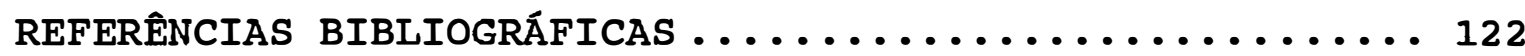

APÊNDICE: Delineamentos compostos pequenos .......... 129 


\title{
AVALIAÇÃO DE ALGUNS DELINEAMENTOS COMPOSTOS PEQUENOS NA EXPERIMENTAÇÃO AGRONÔMICA
}

\author{
Autor: Gener Tadeu Pereira
}

Orientador: Prof. Dr. Dilermando Perecin

\section{RESUMO}

Os delineamentos compostos pequenos com 0 número de observaçōes, $N$, igual ou levemente superior ao número de parâmetros, $p$, do modelo a ser estimado, têm despertado um interesse muito grande por parte de pesquisadores de várias áreas. Este trabalho avaliou as possibilidades destes delineamentos no campo experimental agronômico. Foram estudados os delineamentos compostos pequenos de Hartley, Draper-Lin, Westlake e Lucas, para 4, 5, 6 e 7 fatores e, em duas regiōes experimentais.

os critérios utilizados para esta escolha foram:

(i) possuir tamanho viável no campo experimental agronômico;

(ii) atender aos principais critérios ótimos ( $A, D$ e E) ; 
(iii) ser robusto quanto a má especificação do modelo;

(iv) ser preferencialmente rotacional ou quase-rotacional;

(v) ser preferencialmente ortogonal ou quase-ortogonal;

(vi) possuir uma alta capacidade de reprodução do ponto ótimo (máximo ou mínimo);

(vii) acomodar um sistema simples de blocos ortogonais.

Para a avaliação dos itens (iii) e (vi)

utilizou-se a simulação computacional de dados. Para tanto, cinco situações com formas de modelos reais (simuladores dos dados experimentais) com as respectivas formas dos modelos estimados, foram consideradas nas duas regiões experimentais e para três valores c.v..

As principais conclusões sobre os delineamentos compostos pequenos obtidas neste trabalho foram:

1. Nas situações em que os modelos reais e estimados não diferem na forma, os delineamentos de Hartley e Draper-Lin apresentaram os melhores resultados para os critérios ótimos ( $A, D$ e E), nas duas regiões experimentais $e$, em todas as dimensões consideradas $(k=4,5,6$ e 7$)$, sendo que este desempenho foi melhor ainda na região cuboidal. 0 delineamento de Lucas apresentou o pior desempenho para os três critérios ótimos. Nas situações e nas dimensões em que ocorreram equivalência entre os delineamentos de Hartley e Draper-Lin, a preferência recai sobre o delineamento de Draper-Lin por causa da facilidade de sua obtenção através das colunas dos delineamentos de Placket e Burman.

2. Quando a dimensão aumenta, os delineamentos compostos pequenos tornam-se piores, no sentido dos critérios ótimos. 3. Para $\circ$ número de fatores considerados $(k=4,5,6$ e 7$)$, os delineamentos compostos pequenos possuem um tamanho adequado para experimentos no campo agronômico.

4. A estrutura dos delineamentos compostos pequenos permite 
que estes sejam facilmente arranjados em blocos, sendo que os delineamentos de Lucas e Westlake só podem ser arranjados em, no máximo, dois blocos.

5. A forma da região experimental influi na capacidade de reprodução do ponto crítico dos delineamentos compostos pequenos.

6. Os delineamentos compostos pequenos de Hartley e DraperLin não são robustos quanto a má especificação do modelo, principalmente para altos valores do c.v. e de número de fatores.

7. Para baixos valores do c.v. e na região cuboidal os delineamentos compostos pequenos de Hartley, de Westlake e, principalmente, o delineamento de Lucas são mais robustos à má especificação do modelo quando os dados são simulados a partir de modelos assintóticos e exponenciais, situaçōes 4 e 5, respectivamente.

8. Os delineamentos compostos pequenos estudados neste trabalho não são rotacionais. $\grave{A}$ excessão do delineamento de Lucas, os outros delineamentos podem ser considerados quaserotacionais.

9. As discussōes para a situação 1 permitem afirmar que os delineamentos de Hartley e Draper-Lin são comprometidos com vários critérios. Na região esférica e para pequenos valores de c.v., estes delineamentos têm os melhores resultados para os critérios ótimos, uma boa porcentagem de reprodução do ponto crítico, são quase-rotacionais, e são facilmente arranjados em blocos ortogonais. Na região cuboidal o desempenho destes delineamentos melhora consideravelmente, principalmente, para os três valores do c.v. e para todas as dimensōes. Portanto estes delineamentos podem ser usados no campo experimental agronômico. 


\section{VALUATION OF SOME SMALL COMPOSITE DESIGNS TO AGRONOMIC EXPERIMENTATION}

Author: Gener Tadeu Pereira Adviser: PROF. DR. Dilermando Perecin

\section{SUMMARY}

Small composite designs with the number of trials, $N$, equal or a little greater than the number of parameters, $p$, of the model to be estimated, has aweked a great interest of researchers of many areas. The purpose of this work is the valuation of this designs in agronomic experimentation. The small composite designs studied were Hartley, Draper-Lin, Westlake and Lucas designs, with 4, 5, 6 and 7 factors and, in two experimental regions.

The criterions utilized for the choice of designs was:

(i) possess a practicable size in the agricultural experimentation area;

(ii) attend the main criterions of optimal theory;

(iii) ensure robustness against lack of fit;

(iv) to be preferentialy rotatable or quasi-rotatable;

(v) to be preferentialy orthogonal or quasi-orthogonal; 
(vi) possess a high percentage of reproduction of the optimal point (maximum or minimum);

(vii) allow experiments to be performed in a simple system of blocks.

Computacional simulation of data was utilized for the valuation of (iii) and (vi). So, five situations with forms of real and estimated models was considered.

The main conclusions for the small composite designs from this work are:

1. In situations that there was not differences between the real and estimated model, Hartley designs and Draper-Lin designs had the best values of optimal criterions ( $A, D$ and $E)$, in the two experimental regions, and for all number of factors considered $(k=4,5,6$ and 7$)$, and this performance was better in cuboidal region. Lucas design had the worst performance for all values of optimal criterions in spherical and cuboidal regions. In situations and dimensions that happened equivalence between Hartley and Draper-Lin designs, the recomendation was Draper-Lin designs because it is easily obtained from the colunms of Plackett-Burman designs.

2. When the dimension $\mathrm{k}$ increases, all small composite design studied in this work were worst in the sense of optimal theory.

3. Small composite designs have an adequate size for experiments in agronomic area for $k=4,5,6$ e 7 factors. 4. Small composite designs allow experiments to be performed in a simple system of blocks. Lucas and Westlake designs only could be arranged in two blocks.

5. The form of experimental regions has influence in the capacity of reproducting the critical point of small composite designs.

6. To high values of $\mathrm{c.v}$. and factors the small composite designs showed a fragility in relation to lack of fit. 
7. To small values of $\mathrm{c.v}$. and in the cuboidal region the small composite designs of Hartley, Westlake and, principally, Lucas design showed a best performance of reproduction of point of maximum to data simulated from asymptotic and exponential models, situation 4 and 5, respectivaly.

8. The small composite designs considered in this work are not rotatables. Except for the Lucas design all others designs may be considered quasi-rotatable.

9. The discussion of situation 1 allow to affirm that Hartley and Draper-Lin designs are compromissed with various criterions. In the spherical region and for small values of c.v., this designs had a best performance, for the optimal criterions, a good percentage of reproduction of point of maximum, are quasi-rotatable and are easily arranged in ortogonal blocks. In cuboidal region, this performance increased for all values of c.v. and all dimensions. So this designs may be used in the agronomic experimentation area. 


\section{INTRODUÇÃo}

Frequentemente, pesquisadores de várias áreas, tais como engenharia, química, medicina e agronomia, estudam - comportamento de sistemas em função de modificações nos niveis de certos fatores que influenciam a resposta. o sistema investigado pode ser, por exemplo, um processo de produção industrial, ou um sistema biológico, onde se deseja encontrar, por exemplo, os níveis dos fatores que produzem um comportamento ótimo na resposta. o conjunto de procedimentos, modelos e delineamentos, usados para explorar empiricamente - comportamento da resposta e para definir as condições de operações ótimas do sistema, no qual a resposta é uma variável aleatória, é denominado de Metodologia de Superfície de Resposta (MSR).

A resposta pode ser representada como uma superfície no espaço das variáveis independentes, através de uma função das variáveis ou estímulos presentes no sistema. A verdadeira forma da relação funcional entre a resposta e as variáveis independentes é desconhecida ou complicada. Então, a superfície de resposta é aproximada como uma função simples das variáveis independentes. o modelo serve para descrever, pelo menos em uma região limitada o comportamento da resposta, e para fazer previsão de valores individuais de resposta para uma combinação específica dos níveis das variavéis independentes.

A determinação da natureza da superfície de resposta é acompanhada da experimentação e observação da resposta. A escolha do delineamento experimental tem um 
impacto muito grande na qualidade das inferências obtidas do experimento. Um experimento bem delineado é uma ferramenta valiosa na investigação da resposta de um sistema. Uma das grandes vantagens da MSR está no delineamento; frequentemente, no caso de muitos fatores, é possivel resolver problemas com um número de pontos experimentais, bem menor que por qualquer outra técnica.

o exame do desenvolvimento da teoria dos delineamentos em MSR tem possibilitado a tomada de decisões na construção de delineamentos experimentais para superfícies de resposta. Há várias características que podem ser consideradas na seleção de um delineamento de superfície de resposta. Alguns critérios são discutidos no item 3 deste trabalho. Todos os critérios, pelos quais um delineamento pode ser avaliado, são medidas de um único valor, de alguma característica do delineamento, como por exemplo, a variância generalizada dos coeficientes que estimam $\circ$ modelo da superfície de resposta. Esses critérios têm muita generalidade e concordam em muito com as idéias gerais de eficiência em estatística matemática. Um grande número de critérios foram agrupados sob o titulo geral " $\Phi$ - ótimo ", dentre os quais os critérios D, A, E-ótimos envolvendo requisitos na forma e tamanho do elipsóide da variância das estimativas dos parâmetros. Para vários tipos de modelos, procedimentos analiticos e numéricos foram elaborados para construir delineamentos contínuos ótimos, como por exemplo os delineamentos D-ótimos. O surgimento de delineamentos comprometidos com características ótimas dos delineamentos contínuos foi uma consequência natural da grande influência da teoria dos delineamentos ótimos.

outros critérios, como por exemplo ortogonalidade e rotacionalidade, que não levam a interpretações generalizadas, têm sido considerados e 
exercem, também, um papel importante na decisão do pesquisador.

É consenso em MSR, que a seleção de um delineamento experimental deve ser guiada por mais de um critério. Não se pode esperar que um simples critério forneça um delineamento que satisfaça a todas as caracteristicas em todos os campos de aplicação. Um delineamento pode ser aproximadamente ótimo para um critério, mas muito aquém de um ótimo com respeito a outro. Para modelos suficientemente complicados, como por exemplo os modelos de segunda ordem, critérios, tais como, A, D e E-eficiência, são mutuamente incompativeis, ou seja, em princípio os delineamentos não podem satisfazer a todos estes critérios de eficiência simultaneamente. Tudo que se pode esperar de um delineamento em MSR é que ele tenha um desempenho uniforme para vários critérios que são importantes do ponto de vista do pesquisador. É evidente que a compilação de catálogos de delineamentos e suas caracteristicas, permite ao pesquisador, em MSR, selecionar delineamentos próximos dos ótimos de acordo com muitos critérios e características, simultaneamente. Esta linha de pensamento em MSR mudou completamente o conceito de escolha do critério ótimo para delineamentos. Fica claro que não há necessidade de se escolher um critério e produzir argumentos finais para explicar esta escolha. Assim surgiu a possibilidade de se escolherem delineamentos, suficientemente bons, embora não ótimos, comprometidos com vários critérios.

Como se pode verificar pela literatura, o planejamento de delineamentos experimentais em MSR começou e vem se desenvolvendo extensivamente em um contexto industrial e, com muito menos ênfase, em áreas de pesquisa em agronomia. As limitações e oportunidades da experimentação industrial são tão diferentes da experimentação agronômica, que 
delineamentos apropriados em uma área podem ser totalmente inadequados em outras áreas.

os delineamentos fatoriais clássicos e os fatoriais fracionários têm sido muito estudados e utilizados no campo experimental agrônomico. o inconveniente desses delineamentos é que o número de observações cresce rapidamente em função do número de fatores, o que os tornam inviáveis quando a experimentação é muito cara. os delineamentos compostos pequenos com um número de observações, igual ou levemente superior ao número de parâmetros $\mathbf{p}$ do modelo a ser estimado, tem despertado um interresse muito grande por parte dos pesquisadores em MSR, principalmente no campo agronômico. Um estudo da potencialidade desta classe de delineamentos no campo experimental agronômico se faz necessário.

Assim, tendo em mente estas considerações e que a estimação e análise da superfície de resposta estimada é primordial em MSR, o objetivo deste trabalho foi avaliar, selecionar e catalogar delineamentos compostos pequenos com um número de fatores variando de 4 a 7 , suficientemente bons, não só necessariamente no sentido da teoria do delineamento ótimo, mas também do ponto de vista de vários outros critérios, e com uma alta eficiência prática no campo experimental agrônomico.

os delineamentos, os quais estão descritos na seção 3.7 foram analisados com base nos seguintes critérios:

(i) possuir tamanho viável no campo agronômico;

(ii) atender a critérios da teoria ótima;

(iii) ser robusto quanto a má especificação do modelo e quanto a perda de pontos experimentais;

(iv) ser preferencialmente rotacional ou quase;

(v) ser preferencialmente ortogonal ou quase;

(vi) possuir uma maior capacidade de reproduzir o ponto 
ótimo (máximo ou mínimo).

(vii) acomodar um sistema simples de blocos.

Foram escolhidos os números de fatores de 4 a 7, em virtude da literatura existente dar maior ênfase aos casos de 2 a 3 fatores e existir uma certa dificuldade quando se pretende trabalhar com mais de 3 fatores e mais de 2 niveis. 


\section{REVISÃO DA IITERATURA}

A literatura sobre MSR é extensa. A revisão sobre MSR de MEAD \& PIKE (1975) assinala que as primeiras contribuições importantes, neste campo, apareceram em estudos que envolviam curvas de crescimento para animais e plantas, análises de próbites e, um terceiro campo, onde o termo superficie de resposta apareceu pela primeira vez, foi o relacionamento entre produção de uma cultura e niveis de fertilizantes ou espaçamentos. Segundo MEAD \& PIKE (1975), 0 trabalho pioneiro na quantificação das relações entre crescimento de plantas e fatores ambientais foi feito por MITSCHERLICH. Em sua lei de relações fisiológicas, MITSCHERLICH argumentou que uma relação assintótica entre a produção de uma cultura em função de um fator essencial de crescimento, no caso o espaço, era biologicamente razoável. CROWTHER \& YATES (1941) utilizaram a equação de resposta de MITSCHERLICH, para estudar a resposta de uma cultura arável à vários tipos de fertilizantes.

Embora o planejamento de experimentos para investigar especificamente curvas e superficies não houvesse sido considerado até então, o desenvolvimento de fatoriais complexos por YATES (1935) foi claramente motivado por superficie de resposta e forneceu a base sobre a qual as ultimas pesquisas em planejamento de experimentos em MSR estão assentadas.

Um novo campo de pesquisa em MSR surgiu em decorrência da revolucionária publicação de BOX \& WILSON (1951). Neste artigo eles discutiram planejamento e análise 
de experimentos, com o propósito de encontrar condiçōes ótimas no conjunto das variáveis independentes, ou seja, as condiçōes que fornecem uma resposta ótima, utilizando o menor número possível de observaçōes. A idéia dominante, neste artigo, é que a resposta pode ser aproximada, em uma região de interesse, por um polinômio nos níveis dos vários fatores. Diferentes delineamentos foram comparados em termos da matriz de variância e covariância dos parâmetros a estimar e foi introduzido o conceito de delineamento composto. Eles discutiram, também, o método da máxima inclinação ascendente na determinação de uma região estacionária, próxima de uma resposta ótima.

As primeiras aplicaçōes dos métodos de BOX \& WILSON (1951) foram na Química, pela facilidade de condução de um sistema de experimentos sequenciais. Contudo, eles expressaram a esperança de que os métodos seriam de grande valor em todas as áreas de pesquisa onde a experimentação é sequencial e o erro é razoavelmente pequeno.

Dividindo a esfera de influência com BOX \& WILSON (1951), tem-se o artigo de BOX \& HUNTER (1957). Neste artigo, a seleção do delineamento experimental foi com base na variância da predição $\operatorname{Var}\{\hat{y}(x)\}$. A distribuição da $\operatorname{Var}\{\hat{y}(x)\}$ sobre o espaço das variáveis dependentes foi explorado. Uma propriedade que surgiu naturalmente foi a da rotacionalidade. O delineamento rotacional torna todas as direçōes equivalentes, isto é, a $\operatorname{Var}\{\hat{y}(x)\}$ depende somente da distância de $x$ ao centro do delineamento.

Segundo MYERS et al. (1989), a importância histórica desta propriedade está na necessidade de se obter estabilidade na variância de predição, visto que muitos delineamentos têm uma variância de predição muito instável no perímetro da região do experimento. Ao contrário de muitos outros critérios, rotacionalidade pode ser imediatamente 
utilizada em delineamentos padronizados. Em algumas situaçōes como no início de uma investigação e quando pouco se conhece sobre as variáveis, a propriedade da rotacionalidade pode ser sacrificada em favor de alguma outra propriedade desejada. Entretanto, sempre que for possível, deve-se manter algum grau de rotacionalidade. Três medidas de rotacionalidade foram introduzidas recentemente. KHURI (1988) e DRAPER \& PUKELSHEIM (1990) introduziram medidas quantitativas, as quais são expressas como porcentagem, tomando o valor 100 se e somente se o delineamento é rotacional. A outra medida, apresentada por DRAPER \& GUTTMAN (1988), fornece informação a respeito da forma dos contornos de variância nos delineamentos de segunda ordem simétricos.

outro trabalho motivado pela propriedade da rotacionalidade foi 0 de De BAUN (1959) que mostrou ser possivel construir delineamentos de segunda ordem onde somente três niveis de cada fator são usados. Também dentro desta classe de delineamentos úteis e econômicos no número de pontos experimentais exigidos, estão os delineamentos de BOX \& BEHNKEN (1960).

BOX \& DRAPER $(1959,1963)$ introduziram a noção de robustez de um delineamento de experimento quanto a má especificação do modelo. Mostraram que um critério baseado em $\mathbf{J}$, o desvio médio quadrático da verdadeira resposta integrada sobre alguma região de interesse, $R$, pode ser dividido em duas partes representando o que eles denominaram de "erro de variância", V, e "erro do vício", B, isto é, $\mathbf{J}=\mathbf{V}+\mathbf{B}$. Estes trabalhos forneceram a base da qual muitos outros pesquisadores desenvolveram outros estudos sobre delineamentos de superfície de resposta.

Uma outra linha de pesquisa em MSR, surgiu com a importante série de artigos de KIEFER (1958, 1959, 1960, 1961, 1962 a,b) e KIEFER \& WOLFOWITZ (1959, 1960). Através 
destes artigos, foram lançados os fundamentos para uma rigorosa e sistemática teoria dos delineamentos experimentais ótimos.

A teoria do delineamento ótimo tornou-se um importante componente no desenvolvimento geral do delineamento experimental para modelos de regressão, segundo MYERS et al. (1989). A principal característica dessa teoria é a percepção do delineamento como uma medida de probabilidade com forte ênfase na redução da variância com respeito ao modelo ajustado. O critério mais proeminente desta teoria é o D-ótimo, o qual minimiza a variância generalizada dos coeficientes a estimar com o modelo. Outros critérios, também importantes, são o A-ótimo, que minimiza a variância média dos estimadores dos parâmetros e o critério E-ótimo que minimiza o máximo autovalor da matriz de covariância dos estimadores. Estes critérios estão ligados à moderna teoria da estimação estatística. Por exemplo, de acordo com a teoria estatística geral, usando o método dos mínimos quadrados, pode-se encontrar estimativas conjuntas eficientes dos parâmetros, ou seja, onde a variância generalizada é mínima. Então, é bem lógico exigir que os delineamentos devam minimizar a variância generalizada em um conjunto de delineamentos. Tais delineamentos são denominados D-ótimos. Estes critérios ótimos podem ser considerados como uma evolução a mais do bem conhecido conceito de estimativas conjuntas eficientes.

KIEFER (1958) também generalizou a noção de delineamento. Em particular, ao invés de considerar um delineamento como um conjunto de pontos com um número fixo de observações em cada ponto (delineamento exato), KIEFER sugeriu um " delineamento contínuo " tal como uma medida de probabilidade em um conjunto dado. Usando este conceito de delineamento, KIEFER \& WOLFOWITZ (1960) provaram alguns 
teoremas importantes sobre as propriedades dos delineamentos ótimos e sobre a equivalência de diferentes critérios ótimos. o problema de se construir delineamentos ótimos tornou-se fácil para os delineamentos contínuos e alguns desses delineamentos têm sidos construídos para vários modelos de regressōes particulares.

Uma critica importante à teoria de delineamentos D-ótimos é que ela é apresentada dentro de um esquema matemático rígido, orientada por hipóteses que podem não ter nada com a realidade. Na prática, uma medida D-ótima não tem aplicação imediata, dado que na realização de um experimento deve-se utilizar um delineamento discreto, ou seja um delineamento com um número de pontos experimentais inteiros. Como um resultado desta particularidade dos delineamentos D-ótimos, a teoria dos delineamentos ótimos foi desenvolvida como uma teoria puramente matemática sem preocupação com uso prático. Entretanto, é interessante conhecer quão próximo esse delineamento está da medida ótima. BOX (1978) compilou uma lista, onde os critérios da teoria ótima são unidos àqueles relacionados à minimização dos erros devido a escolha incorreta do modelo, e a muitas outras propriedades desejáveis do delineamento; assegurando com isso sua conveniência na aplicação. Entretanto, na prática, não houve concordância entre os vários critérios. Como regra, delineamentos ótimos, mesmo para regiōes padronizadas e simétricas, exigiam, para sua aplicação prática, arredondamento $e$, consequentemente perda de eficiência.

Uma atenção maior foi dada à construção de delineamentos $\mathrm{D}_{\mathrm{N}}$-ótimos. Este delineamento consiste de $\mathrm{N}$ pontos experimentais, com $\mathrm{N}$ fixo, para o qual o determinante da matriz de informação, para o modelo ajustado, é máximo. vários algoritmos fornecem delineamentos $D_{N}$-ótimos, dentre 
eles, destaca-se o algoritmo DETMAX de MITCHELL (1974), o qual gera uma sequência de $\mathrm{N}$ pontos experimentais com valores do $\left|x^{\prime} \mathbf{x}\right|$ não decrescentes para um valor de $\mathrm{N}$ fixado.

NALIMOV et al. (1970) publicaram um trabalho onde a eficiência dos delineamentos frequentemente utilizados foram comparadas numericamente com respeito a vários critérios. Foi mostrado que quase sempre é possível, com um pequeno número de observaçōes, para modelos e regiōes padronizadas, encontrar, entre os delineamentos simétricos, aqueles com uma eficiência suficientemente alta, isto é, de acordo com o critério D-ótimo.

LUCAS (1974) avaliou o desempenho de alguns delineamentos de superfície de resposta para modelos de segunda ordem através da D- e G-eficiência definidas por ATWOOD (1969).

Com a finalidade de se obter delineamentos com tamanho adequado e maior número de níveis para cada fator, CONAGIN \& JORGE (1977) construiram três fatoriais fracionários $(1 / 5) 5^{3}$, gerados pela superposição de três quadrados latinos ortogonais. Destacaram que esses delineamentos têm um tamanho bastante adequado e um número suficiente de níveis para pesquisas na área agronômica. Com - mesmo objetivo, PERECIN et al. (1982) construiram as 155 fraçōes regulares dos delineamentos fatoriais fracionários $(1 / 5) 5^{3}$, geradas pelo confundimento de componentes ortogonais derivados da teoria de "Campos de Galois ", mostrando que as três fraçōes de CONAGIN \& JORGE (1977) e outras 37 apresentam propriedades semelhantes para serem utilizadas como delineamentos para superfície de resposta. Em continuidade ao trabalho de Perecin et al. (1982), PEREIRA (1984), PEREIRA \& PERECIN (1985) avaliaram as quarenta fraçōes regulares $(1 / 5) 5^{3}$, classificadas em tipos $W, Y$ e $z$, quanto à eficiência dos estimadores dos parâmetros do modelo de segunda ordem, 
quando comparados com o delineamento composto central ortogonal de 15 pontos. As frações tipo $W$ apresentaram a melhor estrutura de confundimento dos parâmetros do modelo polinomial de segunda ordem. Também foram mais eficientes na estimação dos parâmetros deste modelo polinomial.

ANDRADE \& NOLETO (1985) geraram uma série de arranjos fatoriais $(1 / 2) 4^{3}$ e (1/4) $4^{4}$, através da utilização da técnica do confundimento, os quais satisfazem as condições de ortogonalidade entre os efeitos de maior interesse.

SANCHES (1986), utilizou vários delineamentos com 3, 5, 7 e 9 níveis e o modelo polinomial quadrático com três variáveis (fatores) com o objetivo de estudar o problema da grande frequência de ponto de sela na superfície ajustada. Por simulação de dados e utilizando a porcentagem de reprodução de máximo, também fez uma comparação entre os delineamentos utilizados.

Também com o propósito de obter delineamentos de superfícies de resposta no campo agronômico, EDMONDSON (1991) lista as principais diferenças entre a experimentação agronômica e industrial, e com base nestas diferenças construiu delineamentos fatoriais a quatro níveis, utilizando a teoria de confundimento para a obtenção de delineamentos pseudo-fatoriais a dois níveis.

o desenvolvimento de delineamentos compostos pequenos, com um pequeno número de combinações experimentais igual, ou que excede um pouco o número de parâmetros, p, da superfície de resposta, a serem estimados, denominados de saturados ou quase-saturados, respectivamente, progrediu nos anos 70. A motivação de obtenção de uma alta D-eficiência foi a primeira motivação para a construção de novos delineamentos neste período. Para regiões esféricas, os delineamentos de segunda ordem econômicos mais úteis são os delineamentos compostos pequenos e os delineamentos híbridos 
devido a ROQUEMORE (1976). A idéia de um delineamento composto pequeno é devida a HARTLEY (1959), que utilizou a menor fração regular do fatorial $2^{k}$ de resolução III, em substituição às frações de resolução $\mathrm{V}$ ou fatoriais completos na parte cubo do delineamento composto, para obter delineamentos saturados ou quase-saturados para $k=2,3,4$ e 6 fatores. Para $\mathrm{k}=5,6,9$, e números maiores, WESTLAKE (1965) conseguiu uma conveniente melhora utilizando frações irregulares do sistema fatorial $2^{k}$ no núcleo dos delineamentos compostos, ao invés dos fatoriais completos utilizados por BOX \& WILSON (1951) e as frações regulares utilizadas por HARTLEY (1959).

outra alternativa, revolucionária, que facilitou a obtenção de delineamentos compostos pequenos, foi utilizada por DRAPER (1985), o qual empregou colunas do já quase sem uso delineamento de PLACKETT \& BURMAN (1946) na parte cubo dos delineamentos compostos, ao invés de frações regulares ou irregulares. DRAPER \& LIN (1990) apresentaram alternativas nesse processo para $k$ menor ou igual a 10 e recentemente LIN (1993) apresentou uma nova classe de delineamentos super-saturados utilizando meia fração das matrizes de Hadamard.

As revisões bibliográficas sobre MSR exercem um papel importante na compreenssão do assunto. Dentre as revisões destacam-se a de HILL \& HUNTER (1966) com ênfase nas aplicações práticas na área da química e a ampla revisão de MEAD \& PIKE (1975) com ênfase nas aplicações biológicas. Mais recentemente, MYERS et al. (1989) revêem o progresso da MSR nas áreas gerais do delineamento experimental e análise de superficie de respostas. São apontadas as áreas de pesquisa atuais e para futuras pesquisas em MSR.

Dentre os livros textos que tratam em parte, ou na sua totalidade, de delineamentos experimentais para a 
exploração de superfície de respostas estão os de DAVIES (1954), MYERS (1976), BOX \& DRAPER (1987), KHURI \& CORNELL (1987), ATCKINSON \& DONEV (1992), entre outros. 


\section{METODOLOGIA}

\subsection{O modelo matemático do sistema}

Considere que y representa uma resposta a qual se supōe depender dos fatores $\xi_{1}, \xi_{2}, \ldots, \xi_{k}$ presentes no sistema. Também é assumido que o pesquisador tem controle sobre os valores $\xi_{1}, \xi_{2}, \ldots, \xi_{\mathrm{k}}$ no experimento e que estas variáveis são quantitativas contínuas. Assim, pode-se definir o sistema, matematicamente, como

$$
y=g\left(\xi_{1}, \xi_{2}, \ldots, \xi_{k}\right)+\epsilon
$$

onde $\epsilon$ é o termo usual do erro. Como foi mencionado anteriormente, a forma funcional de $g$ é, no geral, desconhecida. As vezes pode ser conveniente expressar 0 modelo em termos de variáveis codificadas $x_{1}, x_{2}, \ldots, x_{k}$ e não em termos das variáveis $\xi_{1}, \xi_{2}, \ldots, \xi_{k}$, as quais são expressas nas unidades originais do sistema. Estas novas variáveis são transformaçōes lineares simples das variáveis originais. Em um experimento onde $N$ medidas da resposta são tomadas em $\xi_{\text {ui }}$ ou seja, no nível do fator $i$ para a u-ésima combinação, $\operatorname{com} u=1,2, \ldots, N$ e $i=1,2, \ldots, k$. A correspondente variável $x_{i}$ é definida por

$$
x_{u i}=\frac{\xi_{u i}-\bar{\xi}_{i}}{S_{i}}, \mathrm{u}=1,2, \ldots, \mathrm{N}
$$

onde $\bar{\xi}_{i}=\sum_{u=1}^{N} \xi_{u i} / N$ e $\boldsymbol{s}_{\mathrm{i}}$ é um termo de escala, convenientemente escolhido. 
Uma prática comum é aproximar $g$ por um polinômio de grau a em alguma região dos valores permitidos das variáveis independentes $\xi_{1}, \xi_{2}, \ldots, \xi_{k}$. o modelo polinomial de primeira ordem para a resposta observada, em termos das variáveis $x_{i}, i=1,2, \ldots, k$, é

$$
y=\beta_{0}+\sum_{i=1}^{k} \beta_{i} x_{i}+\epsilon
$$

o modelo polinomial de segunda ordem é da forma

$$
y=\beta_{0}+\sum_{i=1}^{k} \beta_{i} x_{i}+\sum_{i=1}^{k} \beta_{i i} x_{i}^{2}+\sum_{i=1}^{k} \sum_{\substack{j<1 \\ i<j}}^{k} \beta_{i j} x_{i} x_{j}+\epsilon,
$$

enquanto que

$$
y=\beta_{0}+\sum_{i=1}^{k} \beta_{i} \sqrt{x_{i}}+\sum_{i=1}^{k} \beta_{i i} x_{i}+\sum_{i=1}^{k} \sum_{\substack{j<1 \\ i<j}}^{k} \beta_{i j} \sqrt{x_{i}} \sqrt{x_{j}}+\epsilon
$$

é o modelo em raiz quadrada de segunda ordem.

Modelos polinomiais são aproximaçōes bem irrealísticas sobre o espaço todo, mas seu uso em uma região relativamente pequena em torno do ponto estacionário é aceitável como uma expansão em série de Taylor, aproximando uma função mais complicada.

o modelo polinomial de primeira ordem é frequentemente utilizado em uma região experimental pequena onde nem sempre existe uma grande curvatura na função resposta. Este modelo deve ser usado, localmente, para aproximar a função resposta em um estágio inicial do experimento, para que se possa encontrar uma nova região no espaço das variáveis independentes, onde a resposta é maior ou menor. Em regiōes maiores ou quando a procura é próxima a resposta ótima, um modelo polinomial de segunda ordem é usado para aproximar a função resposta naquela região.

Modelos polinomiais de primeira e segunda 
ordem são simples na forma e quase sempre fornecem aproximações adequadas da verdadeira função resposta em alguma região.

\subsection{O delineamento de tratamentos}

No experimento, as respostas observadas são referentes a $\mathbf{N}$ combinaçōes dos níveis das $\mathrm{k}$ variáveis independentes $x_{1}, x_{2}, \ldots, x_{k}$. o conjunto dessas çombinaçōes dos níveis escolhidos é denominado o delineamento de tratamentos. As combinações dos níveis são denominados de pontos do delineamento ou pontos experimentais ou ainda tratamentos, incluindo entre esses, possíveis repetições de uma mesma combinação de níveis. Neste trabalho para efeitos práticos, - conjunto das combinações dos níveis será designado simplesmente de "delineamento". Colocando-se $x_{u}=\left(x_{u 1}, x_{u 2}, \ldots, x_{u k}\right)^{\prime}$ para $\circ$ u-ésimo tratamento, $u=1,2, \ldots$, N, denomina-se de matriz do delineamento, a matriz $D$ de ordem N $\times$ k onde a u-ésima linha é $x_{u}{ }^{\prime}$, ou seja,

$$
D=\left[\begin{array}{cccc}
x_{11} & x_{12} & \ldots & x_{1 k} \\
x_{21} & x_{22} & \ldots & x_{2 k} \\
\vdots & \vdots & \vdots & \vdots \\
x_{N 1} & x_{N 2} & \cdots & x_{N k}
\end{array}\right]
$$

o delineamento é uma parte importante na investigação da superfície de resposta. A escolha cuidadosa de um delineamento é crucial para a qualidade e para a confiança da informação obtida de um experimento. Vários critérios que um pesquisador deve utilizar na escolha de um delineamento são descritos na seção 3.6 . 
3.3. A notação matricial do modelo

Uma forma conveniente do modelo, que descreve a relação funcional das respostas observadas e as variáveis independentes no experimento pode ser colocada como

$$
\underset{\sim}{y}=X \underset{\sim}{\beta}+\epsilon
$$

onde

$y_{\tilde{\beta}}=\left(y_{1}, y_{2}, \ldots, y_{N}\right)^{\prime}$ é o vetor das respostas observadas,

$\underline{\sim}$ é o vetor de parâmetros $\mathrm{p} \times 1$ que aparece no modelo escolhido e

$\underline{\epsilon}=\left(\epsilon_{1}, \epsilon_{2}, \ldots, \epsilon_{N}\right)^{\prime}$ é o vetor de erros aleatórios correspondentes a $y_{\sim}$.

Para os propósitos desse trabalho, é assumido que os erros são variáveis aleatórias independentes e identicamente distribuídas com média zero e variância $\sigma^{2}$. A matriz $\mathrm{x}$ de ordem $\mathrm{N} \times \mathrm{p}$, depende do delineamento e do modelo e será denominada matriz do modelo. Por exemplo, os parâmetros de um modelo de primeira ordem são $\beta_{0}, \beta_{1}, \ldots, \beta_{k}$. o vetor de parâmetros fica então $\underset{\sim}{\beta}=\left(\boldsymbol{\beta}_{0}, \boldsymbol{\beta}_{1}, \ldots, \boldsymbol{\beta}_{k}\right)^{\prime}$. A matriz $\mathbf{x}$ correspondente a este modelo é:

$$
\left[\begin{array}{ll}
\mathcal{1} & D \\
&
\end{array}\right]
$$

\subsection{A estimação}

Uma solução de mínimos quadrados para o vetor de parâmetros desconhecidos, assumindo um modelo linear, é dada por 


$$
\hat{\boldsymbol{\beta}}=\left(X^{\prime} X\right)^{-1} X^{\prime} \underline{\sim}
$$

A matriz de variância-covariância de $\stackrel{\hat{\beta}}{\hat{\beta}}$ sob a hipótese de que $\epsilon \sim\left(\underline{O}, \sigma^{2} I\right)$, é dada por

$$
\operatorname{Var}(\underset{\sim}{\hat{\beta}})=\sigma^{2}(X X)^{-1}
$$

o vetor de resposta média estimada nos pontos do delineamento é

$$
\hat{\underline{y}}=\left(\hat{y}_{1}, \hat{y}_{2}, \ldots, \hat{y}_{N}\right)^{\prime}=X \underset{\sim}{\hat{\beta}},
$$

e o valor estimado para a resposta média para um $X$ na região experimental de interesse é

$$
\hat{y}(\underset{\sim}{x})=f^{\prime}(\underset{\sim}{x}) \underset{\sim}{\hat{\beta}},
$$

onde $f^{\prime}(x)$ é uma linha dá matriz $x$.

A variância do valor da resposta média predita no ponto $\underset{\sim}{X}$ é dada por

$$
\operatorname{Var}(\hat{y}(\underset{\sim}{x}))=\sigma^{2} f^{\prime}(x)(X X)^{-1} f(\underset{\sim}{x})
$$

Para o modelo de primeira ordem, dado pela equação $(3.1 .3), \underset{\sim}{x}=\left(1, x_{1}, x_{2}, \ldots, x_{k}\right)^{\prime}$ e para o modelo de segunda ordem, dado pela equação (3.1.4), $\underset{\sim}{x}=\left(1, x_{1}, \ldots, x_{k}, x_{1}^{2}, \ldots, x_{k}^{2}, x_{1} x_{k}, \ldots, x_{k-1} x_{k}\right)^{\prime}$.

\subsection{Análise da superfície de resposta ajustada}

Será considerado que um delineamento apropriadamente escolhido foi conduzido em uma região experimental de interesse. As estimativas de minimos quadrados foram obtidas conforme descrição na seção 3.4. A 
resposta média estimada, para um $\underset{\sim}{x}$ qualquer do delineamento, com um modelo polinomial de segunda ordem é:

$$
\hat{y}=\hat{\beta}_{0}+\sum_{i=1}^{k} \hat{\beta}_{i} x_{i}+\sum_{i=1}^{k} \hat{\beta} x_{i i}^{2}+\sum_{i=1}^{k} \sum_{\substack{j=1 \\ i<j}}^{k} \hat{\beta}_{i j} x_{i} x_{j}
$$

Colocando-se

$$
\stackrel{\hat{b}}{b}=\left(\hat{\beta}_{1}, \hat{\beta}_{2}, \ldots, \hat{\beta}_{k k}\right)^{\prime}, \underline{\sim}=\left(x_{1}, x_{2}, \ldots, x_{k}\right)^{\prime} \mathrm{e}
$$

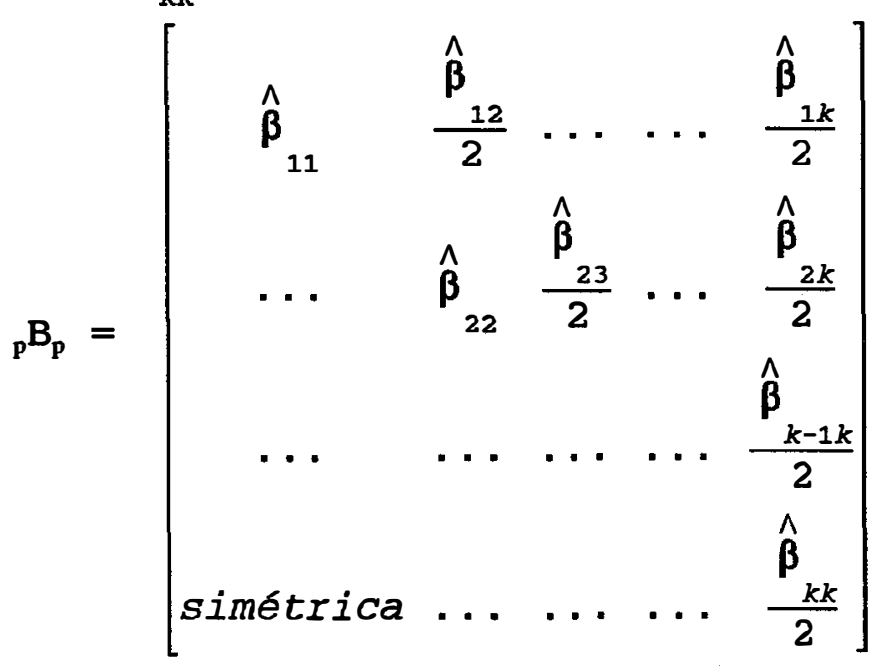

tem-se que

$$
\underset{\sim}{\hat{y}}=\hat{\beta}_{0}+\underset{\sim}{x} \underset{\sim}{b}+\underset{\sim}{x} \cdot \underset{\sim}{X}
$$

o ponto crítico ou ponto estacionário $\underset{\sim}{x^{*}}$ é solução da equação

$$
\frac{\partial \underset{\sim}{y}}{\partial \underset{\sim}{x}}=2 \underset{\sim}{\underset{\sim}{x}} B+\underset{\sim}{b}=0
$$

que é dada por

$$
\underset{\sim}{x^{*}}=-\frac{1}{2} B^{-1} \underset{\sim}{b}
$$

o ponto estacionário ${\underset{\sim}{*}}^{*}$ pode ser: (a) um ponto 
onde a superfície ajustada tem um máximo; (b) um ponto onde a superfície ajustada tem um mínimo; (c) um ponto de sela do modelo ajustado; ou (d) uma indeterminação.

Para se determinar a natureza do ponto estacionário, pode-se analisar a matriz B por meio de seus autovalores. Para isso é conveniente que as equaçōes (3.5.1) e (3.5.2) sejam reescritas em uma forma diferente denominada de forma canônica.

\subsubsection{Redução à forma canônica}

Considere a equação (3.5.1). Suponha que ao invés de se medirem as variáveis a partir do centro do delineamento $(0,0, \ldots .0)$, uma nova origem é considerada no centro do sistema de resposta, ou seja, no ponto estacionário $x_{\sim}^{*}$, e suponha também que em vez de se medirem as variáveis em $\underset{\sim}{x}$, elas sejam medidas em novos eixos definidos por $\underset{\sim}{Z}=\underset{\sim}{x}-{\underset{\sim}{x}}^{*}$. Então a equação (3.5.2) em termos deste novo vetor fica

$$
\hat{y}=\hat{y}_{0}+\underset{\sim}{Z} B \underset{\sim}{Z}
$$

onde $\hat{y}_{0}$ é a resposta estimada em $\underline{\sim}^{*}$. Considerando-se, então, a transformação ortogonal

$$
\underset{\sim}{Z}=M \underset{\sim}{W}
$$

onde $W^{*}=\left(w_{1}, w_{2}, \ldots, w_{k}\right)^{\prime}$ e tal que

$$
M^{\prime} B M=\operatorname{diag}\left(\lambda_{1}, \lambda_{2}, \ldots, \lambda_{k}\right)
$$

Então

$$
\underline{Z}^{\prime} B \underset{\sim}{Z}=\lambda_{1} w_{1}^{2}+\lambda_{2} w_{2}^{2}+\ldots+\lambda_{k} w_{k}^{2}
$$

onde $\lambda_{1}, \lambda_{2}, \ldots, \lambda_{k}$ são os autovalores da matriz B. M é uma 
matriz ortogonal $k \times k$, ou seja, $M^{\prime} M=I_{k}$.

Assim a equação (3.5.1.1) fica

$$
\hat{y}=\hat{y}_{0}+\lambda_{1} w_{1}^{2}+\ldots+\lambda_{k} w_{k}^{2}
$$

a qual é a forma canônica do modelo de segunda ordem em $\mathrm{k}$ variáveis. BOX \& DRAPER (1987) denominam a equação (3.5.1.4) como da forma canônica tipo B. A forma canônica do tipo B refere-se a equação (3.5.1) nos novos eixos $\left(w_{1}, w_{2}, \ldots, w_{k}\right)$. Assim, para determinar a natureza do ponto estacinário, basta analisar os autovalores. Se os autovalores:

(i) são todos negativos, então a solução é um máximo;

(ii) são todos positivos, então a solução é um mínimo;

(iii) têm sinais misturados, então tem-se um ponto de sela;

(iv) contém zeros, então tem-se uma indeterminação. A indeterminação do item (iv) é no sentido de que a resposta não muda de valor quando se desloca do ponto estacionário $x^{*}$ na direção do eixo $w_{i}$. Podem existir infinitos máximos, ou infinitos mínimos ao longo do eixo $w_{i}$, etc.

A equação (3.5.1.4) na forma matricial fica

$$
\hat{y}=\stackrel{\wedge}{y_{0}}+w \Omega w
$$

onde $\Omega=\operatorname{diag}\left\{\lambda_{1}, \lambda_{2}, \ldots, \lambda_{k}\right\}$ e, de acordo com o descrito na seção anterior, se $\lambda_{i}<0, i=1, \ldots, k$, então $\hat{y}_{0}=\operatorname{máx} \hat{y}_{\sim}$, atingido em $W=0$. Considerando, ainda, a transformação definida na equação (3.5.1.2), tem-se

$$
\underset{\sim}{X}-\underset{\sim}{x^{*}}=M \underset{\sim}{W} \Leftrightarrow=M^{\prime}\left(\underset{\sim}{X}-{\underset{\sim}{x}}^{*}\right)
$$

Então a equação (3.5.1.5) fica 


$$
\hat{\underline{y}}=\hat{y}_{0}+\left(\underline{x}_{-}-\underline{x}^{*}\right)^{\prime} M \Omega M^{\prime}\left(\underline{x}-\underline{x}^{*}\right) .,
$$

a qual é reescrita na forma

$$
\hat{\underline{y}}=\hat{y}_{0}+\left(\underline{\sim}-x^{*}\right)^{\prime} Q\left(\underset{\sim}{x}-x^{*}\right) \text {, }
$$

ou ainda

$$
\hat{y}=\hat{y}_{0}+x^{* \prime} Q x^{*}-2 x^{*} Q x+\underset{\sim}{x^{\prime}} Q x
$$

Esta última forma será utilizada na seção 3.8.2, para obtenção de modelos para simulação de dados.

3.6. Algumas propriedades e critérios de escolha de delineamentos.

Pode-se escolher um delineamento experimental através de vários critérios. Geralmente, algumas propriedades importantes são escolhidas, e seleciona-se o delineamento que atende a estas propriedades. Muitas vezes, diferentes critérios podem levar à diferentes delineamentos. 0 delineamento ou o critério utilizado deve estar de acordo com o objetivo do experimento. Em algumas circunstâncias vários critérios são considerados conjuntamente.

BOX \& DRAPER $(1975,1987)$ listam 14 objetivos na escolha de um delineamento experimental. Algumas, todas ou qualquer uma dessas propriedades podem ser importantes. Assim, segundo esses autores, o delineamento tem que:

1. Gerar uma distribuição de informação satisfatória na região de interesse, a qual pode não coincidir com a região do delineamento (região experimental);

2. Assegurar que o valor ajustado $\hat{y}(x)$ em $\underset{\sim}{X}$, esteja o mais próximo possível do verdadeiro valor médio $\mathrm{E}(\mathrm{y})$;

3. Tornar possível a checagem da falta de ajuste;

4. Permitir a estimação de transformações de fatores experimentais quantitativos;

5. Permitir que experimentos sejam conduzidos em blocos;

6. Permitir o aumento da ordem do delineamento para ser 
construído sequencialmente;

7. Fornecer uma estimativa do erro através de repetição;

8. Ser insensível a observaçōes aberrantes e a violaçōes das hipóteses usuais da teoria normal;

9. Requerer um número pequeno de pontos experimentais;

10. Fornecer padrōes de dados simples permitindo uma rápida apreciação visual;

11. Assegurar simplicidade de cálculos;

12. Comportar-se bem quando erros ocorrem no conjunto das variáveis experimentais;

13. Não requerer um número grande de níveis dos fatores experimentais;

14. Fornecer uma verificação na hipótese da homocedasticidade.

Esta lista de objetivos pode ser aplicada para a maioria dos experimentos. Dois objetivos a mais, que podem ser importantes para experimentos com fatores quantitativos são os seguintes:

15. Ortogonalidade - os delineamentos têm uma matriz de informação (X'X) diagonal, levando à estimativas não correlacionadas dos parâmetros;

16. Rotacionalidade - a variância de $\hat{y}(x)$ depende somente da distância do ponto $\underset{\sim}{X}$ ao centro do delineamento.

ortogonalidade é uma exigência muito restritiva de ser atendida na maioria dos delineamentos considerados nesse trabalho. Entretanto, ela é a propriedade mais comum dos delineamentos fatoriais $2^{k}$ e delineamentos para fatores qualitativos. Atualmente, com as facilidades computacionais a exigência de ortogonalidade não é tão fundamental como antigamente, ver discussão em MEAD (1990). Rotacionalidade foi muito utilizada por BOX \& DRAPER (1963) na construção de delineamentos para modelos de segunda ordem e, atualmente, é possível determinar quanto rotacional é o 
delineamento (ver seção 3.6.5.1.).

Como foi mencionado anteriormente, a resposta é modelada através de uma função linear ou quadrática das variáveis independentes ou (fatores presentes no sistema). Em todos os casos, o objetivo é obter o melhor ajuste para a resposta. o delineamento escolhido fornecerá a melhor estimativa dos verdadeiros parâmetros no modelo selecionado, ou seja, um bom ajuste do modelo minimizará a variância dos coeficientes estimados.

Uma apresentação mais formal da teoria do delineamento ótimo é feita a seguir.

\subsubsection{Delineamentos contínuos e exatos.}

Nesta seção é feita uma apresentação de alguns aspectos da teoria do delineamento ótimo. Uma afirmação mais formal da importante distinção entre delineamentos contínuos e delineamentos exatos, com uma notação própria, se faz necessária.

Como colocação do problema, considere o seguinte exemplo: um delineamento D-ótimo a dois níveis, para - modelo linear envolvendo um fator com um número de observações $\mathbf{N}$ par, tem metade das observações colocadas em $\mathrm{x}$ $=-1$ e a outra metade em $x=+1$, quando a região experimental é $[-1,+1]$. Para um número de pontos amostrais ímpar, a divisão é feita a mais balanceada possível. Então, para valores de $\mathrm{N}$, ímpares e crescentes, existirá uma sequência de delineamentos exatos, começando-se com a divisão dos pontos 2 para 1 quando $\mathrm{N}=3$, a qual se aproxima da divisão para $\mathrm{N}$ par. O problema matemático de se encontrarem delineamentos ótimos é simplificado quando se considera apenas esta 
aproximação assintótica ou delineamentos contínuos, ignorando-se, então, a restrição de que o número de pontos experimentais em qualquer delineamento deve ser um inteiro. Delineamentos contínuos são representados pela medida $\zeta$ sobre a região experimental $R$. Se o delineamento tem $\mathbf{N}$ pontos experimentais distintos em $R$, tem-se

$$
\zeta=\left\{\begin{array}{lll}
x_{1} & x_{2} \ldots & x_{n} \\
w_{1} & w_{2} \ldots & w_{n}
\end{array}\right\}
$$

onde a primeira linha dá os valores dos fatores nos pontos do delineamento e os $w_{i}$ são pesos correspondentes às proporções destes pontos. Dado que $\zeta$ é uma medida, então

$$
\int_{R} \zeta(d x)=1 \text { e } 0 \leq w_{i} \leq 1
$$

Então, o delineamento D-ótimo do exemplo descrito pela equação (3.6.1.1), nesta notação fica

$$
\zeta=\left\{\begin{array}{cc}
-1 & +1 \\
\frac{1}{2} & \frac{1}{2}
\end{array}\right\},
$$

enquanto que 0 delineamento D-ótimo para 3 pontos experimentais fica

$$
\zeta=\left\{\begin{array}{cc}
-1 & +1 \\
\frac{1}{3} & \frac{2}{3}
\end{array}\right\},
$$

e a notação geral para uma medida de um delineamento exato, realizado para um $\mathbf{N}$ inteiro específico, é dada por

$$
\zeta_{N}=\left\{\begin{array}{cccc}
x_{1} & x_{2} & \ldots & x_{n} \\
I_{1 / N} & I_{2 / N} & \ldots & r_{n / N}
\end{array}\right\}
$$

onde $r_{i}$ é o número inteiro das repetições em $x_{i}$ e $\sum_{i=1}^{n} r_{i}=N$.

Na prática, todos os delineamentos são exatos. 
Para N moderado, bons delineamentos exatos podem, frequentemente, ser encontrados por aproximações inteiras da medida ótima contínua $\zeta^{*}$. Geralmente, para modelos simples com p parâmetros, existirão p pontos experimentais com igual peso $1 / p$, tal que o delineamento exato $\operatorname{com} N=p$ combinações é ótimo. Entretanto, se os pesos do delineamento, wi, não são racionais, não será possível encontrar um delineamento exato o qual seja idêntico ao delineamento ótimo.

As dificuldades em se achar um delineamento exato, surgem quando $\mathrm{N}$ é próximo ao número de pontos suporte do delineamento contínuo ótimo, levando a uma pobre aproximação de $\zeta^{*}$.

Para um delineamento de tamanho $\mathrm{N}$ a matriz de informação para $\underline{\sim}_{\sim}$ no modelo $E(\underset{\sim}{Y})=X \underline{\sim}$ é definida como

$$
X^{\prime} X=\sum_{i=1}^{N} f\left(x_{i}\right) f^{\prime}\left(x_{i}\right)
$$

e $f^{\prime}\left(x_{i}\right)$ é a i-ésima linha de $x$. Para o delineamento contínuo ótimo $\zeta$, a matriz de informação é

$$
\begin{aligned}
M(\zeta) & =\int_{x} m(x) \zeta(d x)=\int_{x} f(x) f^{\prime}(x) \zeta d x \\
& =\sum_{i=1}^{N} f\left(x_{i}\right) f^{\prime}\left(x_{i}\right) w_{i}
\end{aligned}
$$

A última equação é uma soma sobre os $\mathrm{N}$ pontos do delineamento, que devido a presença dos $w_{i}$, torna-se uma versão padronizada de $(3.6 .1 .4)$ para o delineamento exato $\zeta_{\mathrm{N}}$, isto é,

$$
M\left(\zeta_{N}\right)=\frac{X^{\prime} X}{N}
$$

A variância da resposta predita para um delineamento de tamanho $\mathrm{N}$ é dado pela equação (3.4.5). Para 
os delineamentos contínuos a variância padronizada da resposta é

$$
d(x, \zeta)=f^{\prime}(x) M^{-1}(\zeta) f(x)
$$

uma função do delineamento e do ponto onde a previsão é feita. Se o delineamento é exato

$$
d\left(x, \zeta_{N}\right)=f^{\prime}(x) M^{-1}\left(\zeta_{N}\right) f(x)=\frac{N \operatorname{Var}\{\hat{y}(x)\}}{\sigma^{2}}
$$

a qual é utilizada na comparação de delineamentos.

\subsubsection{Aspectos teóricos dos critérios ótimos.}

o mais importante critério da teoria ótima de delineamentos em aplicação é o critério D-ótimo, o qual minimiza a variância generalizada dos estimadores dos parâmetros. Dois outros critérios que possuem interpretaçōes em termos da matriz de informação $M(\zeta)$ são os critérios A e E-ótimos. No critério A-ótimo $\operatorname{tr}\left\{M^{-1}(\zeta)\right\}$, a variância média das estimativas dos parâmetros é minimizada. No critério E-ótimo a variância do contraste $\underset{\sim}{a} \boldsymbol{\beta}$ é minimizada sujeita à restrição $a^{\prime} a=1$.

Na prática, trabalha-se com $M\left(\zeta_{N}\right)$ definida pela equação $(3.6 .1 .7)$. Os elementos $\left\{C_{i j}\right\}$ de $\left(X^{\prime} X\right)^{-1}$ são proporcionais às variâncias e covariâncias dos estimadores de mínimos quadrados de $\beta_{\sim}$. Assim, o problema do delineamento experimental é o da escolha de um delineamento tal que os elementos $\left\{C_{i j}\right\}$ atendam aos objetivos da pesquisa. Existem $\frac{1}{2} p(p+1)$ destes coeficientes e uma simplificação se faz necessária. 
Uma motivação para simplificação é fornecida pela consideração da região de confiança para $\beta$.

$$
\left(\underline{\boldsymbol{\beta}}-\hat{\boldsymbol{\beta}}^{\boldsymbol{\beta}}\right)^{\prime}\left(X^{\prime} X\right)\left(\underline{\boldsymbol{\beta}}-\hat{\boldsymbol{\beta}}_{\sim}\right) \leq p S^{2} F_{p, v, \alpha}
$$

onde $\boldsymbol{s}^{2}$ é uma estimativa de $\boldsymbol{\sigma}^{2}$ com $\mathbf{v}$ graus de liberdade e $F_{p, v, \alpha}$ é o ponto $\alpha$ o da distribuição $F$ com $p$ e $v$ graus de liberdade.

No espaço p-dimensional, (3.6.2.1) define um elipsóide p-dimensional. o delineamento D-ótimo minimiza o conteúdo desta região de confiança e, consequentemente, minimiza o volume deste elipsóide. Outras propriedades da região de confiança podem ser de interesse. Um elipsóide longo e fino orientado ao longo ou próximo dos eixos dos parâmetros resultará em pobre estimação, comparativamente, de um ou mais parâmetros, isto é, suas variâncias serão grandes. Se o elipsóide é orientado com um apreciável ângulo com os eixos, a variância de cada estimativa individual dos parâmetros pode ser pequena. Entretanto, devido a correlação entre as estimativas, existirá contrastes envolvendo os parâmetros, correspondendo às direçōes dos eixos longos do elipsóide, que serão estimados com imprecisão. Estas são as situaçōes referenciadas pelos critérios $A$ e E-ótimos, respectivamente.

Estas idéias podem ser colocadas mais formalmente considerando-se os autovalores $\lambda_{1}, \lambda_{2}, \ldots, \lambda_{p}$ de $M(\zeta)$ - Os autovalores de $M^{-1}(\zeta)$ são então $1 / \lambda_{1}, 1 / \lambda_{2}, \ldots, 1 / \lambda_{p}$ e são proporcionais ao quadrado do comprimento dos eixos principais do elipsóide de confiança. Então, os três critérios são:

A- ótimo minimiza a soma (ou a média) das variâncias dos estimativas dos parâmetros, ou seja 


$$
\min \sum_{i=1}^{p} \frac{1}{\lambda_{i}}
$$

D-ótimo minimiza a variância generalizada das estimativas dos parâmetros, ou seja,

$$
\min \prod_{i=1}^{p} \frac{1}{\lambda_{i}}
$$

E-ótimo minimiza a variância da estimativa do contraste $\boldsymbol{a}_{\sim}^{\prime} \boldsymbol{\beta}_{\sim}$, com $a^{\prime} a=1$, ou seja,

$$
\min \max \left(\frac{1}{\lambda_{i}^{-}}\right)
$$

Todos os três critérios podem ser considerados como casos especiais do critério mais geral

$$
\Psi_{k}(\zeta)=\left(p^{-1} \sum_{i=1}^{p} \lambda_{i}^{-1}\right)^{1 / k} \quad(0 \leq k<\infty)
$$

Para os critérios A, D, e E-ótimos, os valores de $\mathrm{k}$ são 1, 0 e infinito, respectivamente, quando as operaçōes limites são apropriadamente definidas.

outro critério introduzido por KIEFER \& WOLFOWITZ (1960) e que também faz parte do alfabeto dos critérios ótimos é o critério G-ótimo, o qual é definido por

$$
G=\min m a ́ x \sigma^{2}{\underset{\sim}{x}}^{\prime}\left(X^{\prime} X\right)^{-1} \underset{\sim}{X},
$$

ou seja, minimiza o máximo valor da variância definida pela equação (3.4.5), dentro da região experimental. Este critério está relacionado à qualidade do valor predito da resposta dentro da região experimental. Os autores também foram capazes de estabelecer a equivalência entre os critérios Dótimo e o G-ótimo através do seguinte teorema para um delineamento contínuo. 
Teorema : 0 delineamento $\zeta^{*}$ é D-ótimo se e somente se ele é G-ótimo e se e somente se

$$
\max _{x} d\left(x, \zeta^{*}\right)=p
$$

(onde $\mathrm{p}$ é o número de parâmetros desconhecidos da regressão); isto significa que as seguintes condiçōes

$$
\begin{gathered}
\left|M\left(\zeta^{*}\right)\right|=\max _{\zeta}|M(\zeta)| \\
\max _{x} d\left(x, \zeta^{*}\right)=\min _{x} \max _{x} d(x, \zeta) \\
\max _{x} d\left(x, \zeta^{*}\right)=p
\end{gathered}
$$

são equivalentes.

As matrizes de informação de todos os delineamentos D-ótimos do mesmo problema coincidem. Máximo de $d\left(x, \zeta^{*}\right)$ é alcançado nos pontos do delineamento.

Essas propriedades dos delineamentos D-ótimos contínuos tornam a solução do problema muito mais fácil. A possibilidade de se encontrar um delineamento D-ótimo o qual é concentrado em um número finito de pontos foi provada; o número de pontos não é maior que $p(p+1) / 2$. Para alguns modelos polinomiais é possível encontrar um delineamento Dótimo com um número de pontos não superior a

$$
\left(\begin{array}{c}
n+2 d \\
2 d
\end{array}\right)
$$

onde d é o grau do polinômio.

outro aspecto importante da teoria do delineamento ótimo é que ela está fundamentada na hipótese de que a função polinomial é a verdadeira função resposta. BoX \& DRAPER (1987), ao contrário, consideram que a função 
polinomial deve ser considerada como uma função matemática, a qual se aproxima localmente, da verdadeira, mas desconhecida, função resposta. Duas fontes de erros são identificadas nesta aproximação: o erro aleatório, e o erro sistemático (vício). A teoria do delineamento ótimo só leva em conta o erro aleatório, ignorando o vício.

Como exemplos ilustrativos do comportamento da região de confiança definida pela equação (3.6.2.1) e da variância padronizada da resposta predita, definida pela equação (3.6.1.9), considere os 8 delineamentos apresentados na Tabela 1. Os seis primeiros delineamentos são comparados para o ajuste de um modelo de primeira ordem em um fator quantitativo. Este exemplo foi extraído de ATKINSON \& DONEV (1992).

Suponha que o modelo ajustado é

$$
\hat{y}(x)=16+7.5 x,
$$

e que os gráficos de contôrno para estimativas do parâmetro $\underline{\beta}$, dadas por

$$
(\underline{\boldsymbol{\beta}}-\hat{\boldsymbol{\beta}})^{\prime}\left(X^{\prime} X\right)\left(\underset{\sim}{\boldsymbol{\beta}}-\underline{\hat{\beta}^{\prime}}\right)=1 \text {, }
$$

são apresentados nas Figuras 1 a 6 para os seis primeiros delineamentos da Tabela 1. Este conjunto de contornos de elipses estão centrados em $\hat{\beta}=(16,7,5)^{\prime}$.

A comparação das Figuras 1 e 2 mostra que 0 aumento do tamanho do delineamento diminui o tamanho do elipsóide de confiança. Como se nota, o delineamento 2 é o delineamento 1 repetido duas vezes. Estes delineamentos são ortogonais, então X'X é diagonal e os eixos das elipses são paralelos aos eixos coordenados. 
TABELA 1 -

Alguns delineamentos para modelos de primeira e segunda ordem quando o número de fatores $\mathrm{k}=1$.

\begin{tabular}{cccccccccc}
\hline Delineamento Tamanho $N$ & \multicolumn{7}{c}{ Valores de $\mathrm{x}$} \\
\hline 1 & 3 & -1 & 0 & 1 & & & & \\
2 & 6 & -1 & -1 & 0 & 0 & 1 & 1 & & \\
3 & 8 & -1 & -1 & -1 & -1 & -1 & -1 & 1 & 1 \\
4 & 5 & -1 & $-0,5$ & 0 & 0,5 & 1 & & & \\
5 & 7 & -1 & -1 & $-0,9$ & $-0,85$ & $-0,8$ & $-0,75$ & 1 \\
6 & 2 & -1 & 1 & & & & & & \\
7 & 4 & -1 & -1 & 0 & 1 & & & & \\
8 & 4 & -1 & 0 & 0 & 1 & & &
\end{tabular}

FIGURA 1 - Elipse de confiança definida por $(\underline{\sim}-\hat{\beta})^{\prime}\left(X^{\prime} X\right)(\underline{\beta}-\hat{\beta})=1$ para o delineamento 1 da Tabela 1 quañdo o ajuste é uma regressão simples. Esta figura surge de um delineamento simétrico.

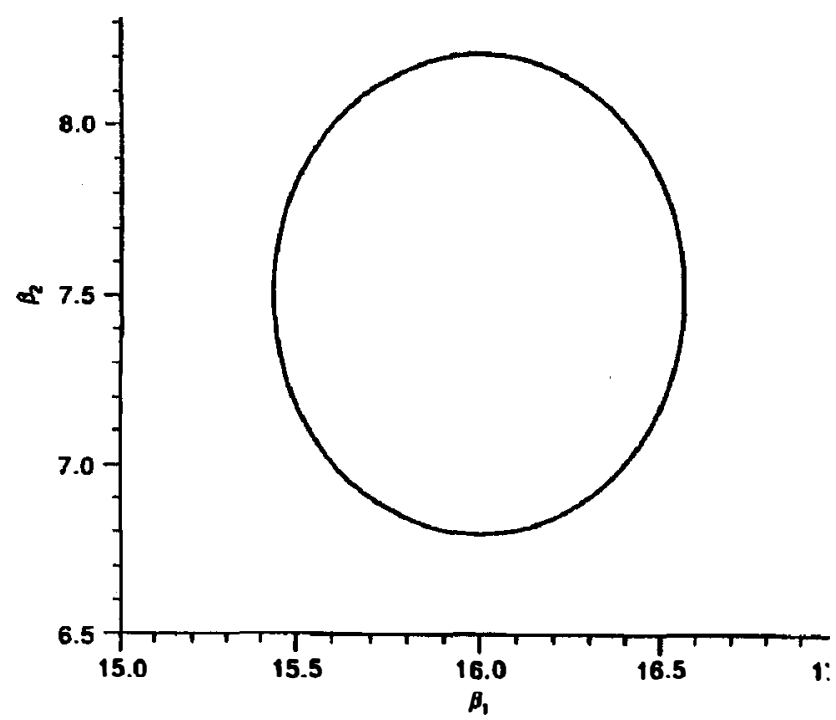


Elipse de confiança para o delineamento 2 da Tabela 1. Esta figura surge de um delineamento simétrico.

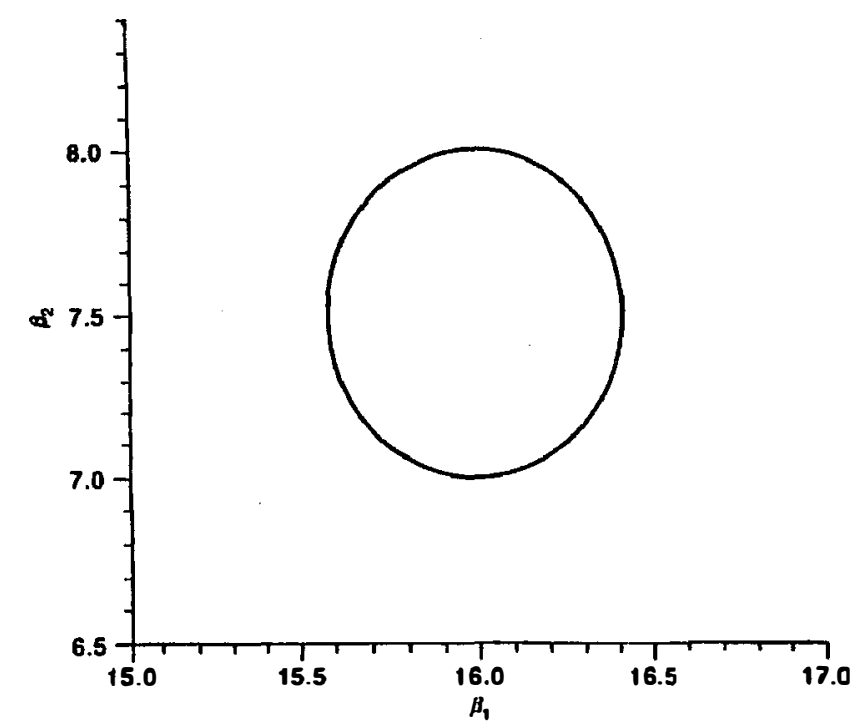

Os delineamentos nas Figuras 3 e 4 têm vários pontos experimentais próximos ao ponto correspondente ao menor nível. Como consequência o delineamento não é ortogonal, ou seja, a matrix $\mathbf{x}^{\prime} \mathbf{x}$ tem elementos diferentes de zero, fora da diagonal e os eixos das elipses não são paralelos aos eixos do sistema de coordenadas. Os eixos destas elipses de confiança têm diferentes tamanhos. A consequência disso é que alguns parâmetros são estimados, com pequena variância e outros são estimados com muito menos precisão. 
FIGURA 3 -

Elipse de confiança para o delineamento 3 da Tabela 1 .

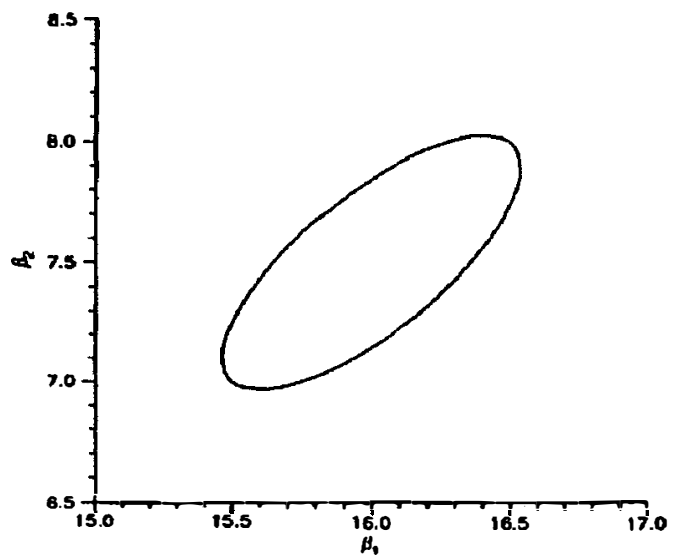

FIGURA 4 -

Elipse de confiança para o delineamento 5 da Tabela 1 .

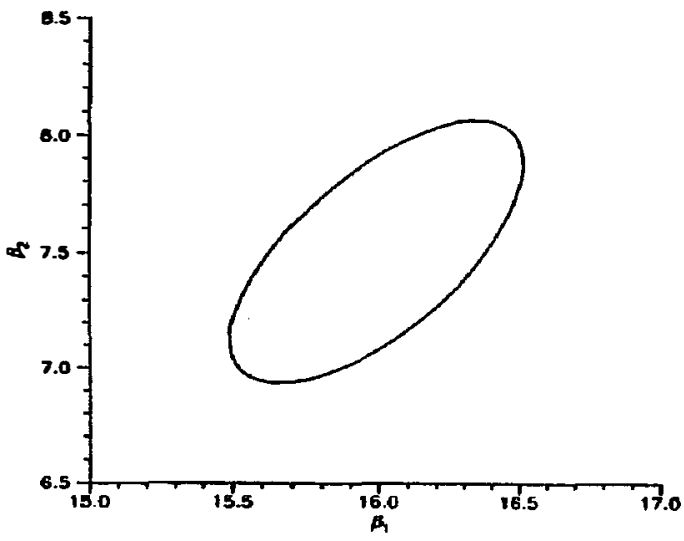


Elipse de confiança para o delineamento 4 da Tabela 1. Esta figura surge de um delineamento simétrico.

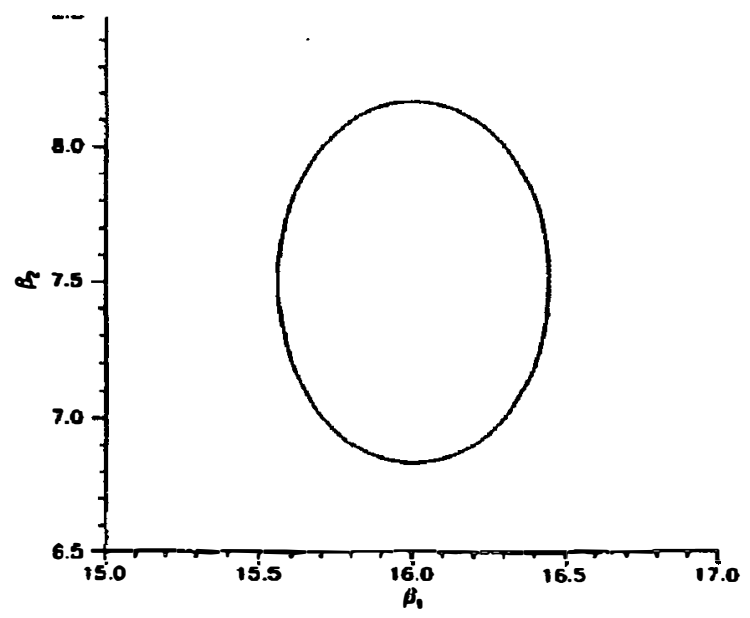

FIGURA 6 -

Elipse de confiança para o delineamento 6 da Tabela 1. Esta figura surge de um delineamento simétrico.

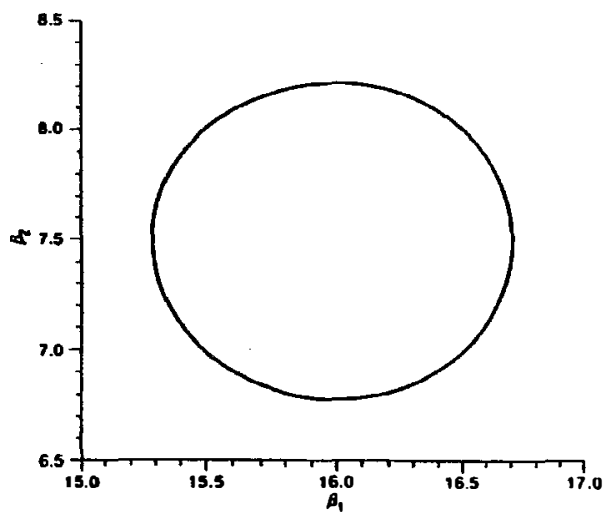


Os delineamentos nas Figuras 5 e 6 são ortogonais. o delineamento $6 \mathrm{com}$ dois pontos experimentais produz a maior região entre todas as outras, pois ele é o delineamento de menor tamanho.

Quando mais de dois parâmetros são importantes, as elipses são substituídas por elipsóides, e o método gráfico utilizado é substituído por métodos analiticos. Geralmente, os eixos do elipsóide são proporcionais às raízes quadradas dos autovalores de $\left(X^{\prime} \mathbf{X}\right)^{-1}$. Um delineamento onde os autovalores diferem apreciavelmente produzem elipsóides longos e finos. O determinante de x'X é proporcional ao produto dos autovalores de $\mathbf{x}^{\prime} \mathbf{x}$. Portanto, em termos destes autovalores um bom delineamento deve ter um grande produto, produzindo uma região de confiança menor, com todos os valores individuais razoavelmente iguais.

As Figuras de 7 a 12 mostram o comportamento da variância padronizada da resposta predita $d(\underset{\sim}{x}, \zeta)$, definida pela equação 3.6.1.9, dos delineamentos da Tabela 1 . Os delineamentos simétricos $1,2,4$ e 6 fornecem gráficos simétricos sobre a região experimental $[-1,1]$. Dado que a variância é padronizada pelo tamanho do delineamento, as Figuras 7 e 8 são iguais. Dos delineamentos simétricos, a Figura 10 mostra o maior valor da variância padronizada da resposta predita sobre a região experimental, exceto para $d(0, \zeta)$, o qual é a unidade para os quatro delineamentos simétricos. Os gráficos nas Figuras 9 e 11 ilustram como o aumento do número de pontos experimentais em uma área da região experimental, no caso próximo a $x=-1$, reduz a variância nesta área, mas produz variância alta em outras áreas. O delineamento 4 tem seus cinco pontos experimentais espalhados uniformemente na região experimental, mas como a Figura 10 mostra, ele não fornece estimativas de $\hat{y(x)}$ com menor variância sobre 
FIGURA 7 - Variância padronizada $d(x, \zeta)$ para 0 delineamento 1 da Tabela 1 para o modelo de primeira ordem em um fator.

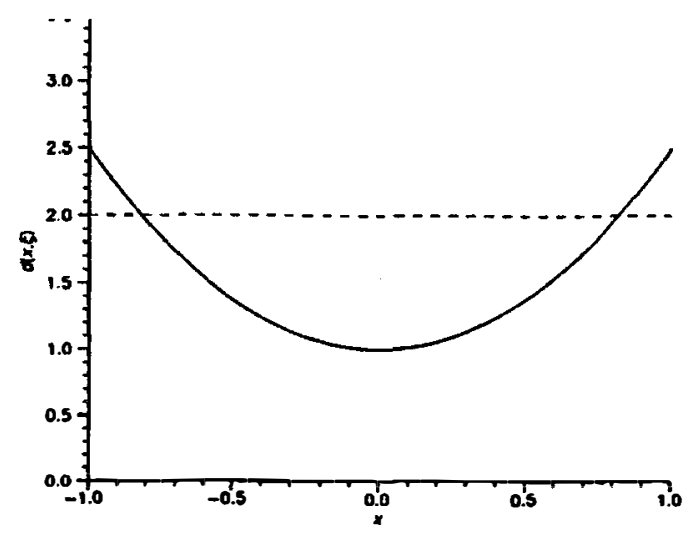

FIGURA 8 - Variância padronizada $d(x, \zeta)$ para 0 delineamento 2 da Tabela 1 para 0 modelo de primeira ordem em um fator.

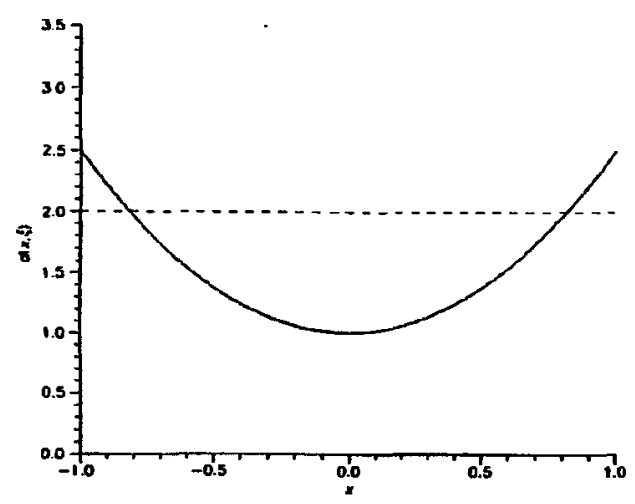


FIGURA 9 -

Variância padronizada delineamento 3 , o qual possui vários pontos experimentais próximos a -1 .

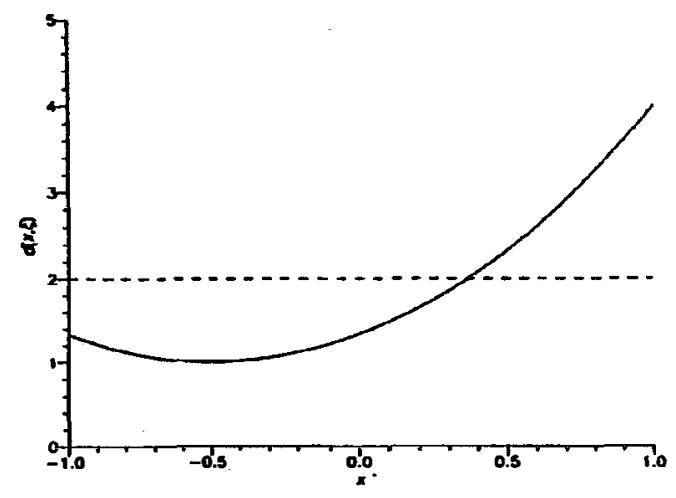

FIGURA 10 - Variância padronizada $d(x, \zeta)$ para o delineamento 4 da Tabela 1 para modelos de primeira ordem em um fator.

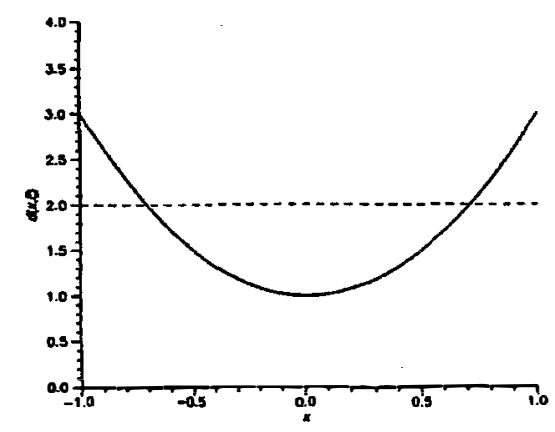


FIGURA 11 - Variância padronizada $\mathrm{d}(\mathrm{x}, \zeta)$ para 0 delineamento 5 da Tabela 1 com ajuste de um modelo de primeira ordem. Delineamento 5 tem vários pontos próximos a -1 .

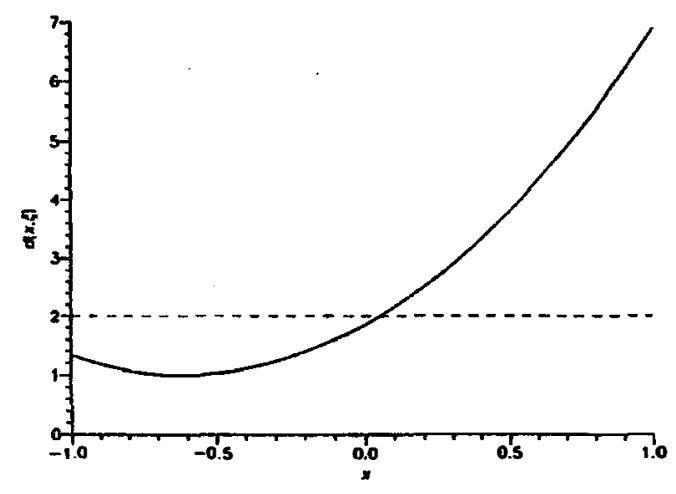

FIGURA 12 - Variância padronizada $d(x, \zeta)$ para 0 delineamento 6 da Tabela 1 com ajuste de um modelo de primeira ordem. Delineamento 6 é D/G-ótimo; o máximo valor de $d(x, \zeta)$ é 2 .

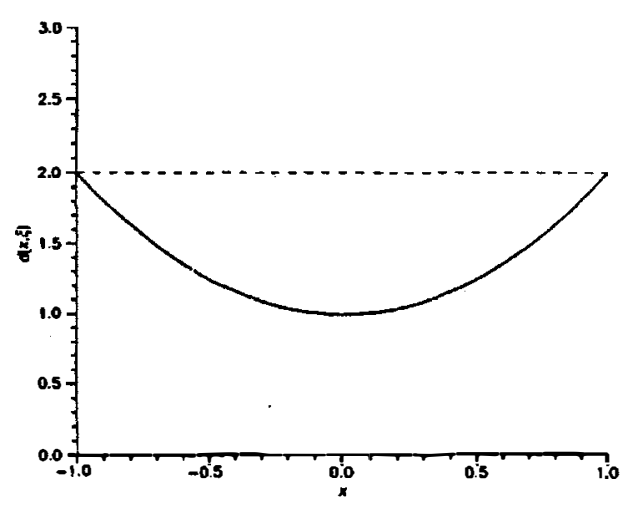


toda a região.

A Figura 12 mostra que o delineamento 6, 0 qual tem numeros iguais de pontos experimentais nos extremos da região e nenhum no outro, fornece o delineamento com menor $d(x, y)$ sobre toda a região experimental.

Estes exemplos mostram como diferentes delineamentos podem ser em termos dos seus valores de $\left|x^{\prime} \mathbf{x}\right|$, em termos da curva de $\mathbf{d}(x, \zeta)$ sobre a região experimental, $e$ em termos do valor máximo da variância sobre a região experimental. Um delineamento ideal para estes critérios deveria, simultaneamente, minimizar a variância do estimador do modelo e minimizar $\mathbf{d}(\mathbf{x}, \zeta)$ sobre a região experimental. os critérios D, A, E e G-ótimos procuram refletir esta situação.

\subsubsection{Critério da variância da predição}

A capacidade de predição de um delineamento é de particular importância em problemas de superfície de resposta. Como foi dito, a MSR está, a princípio, preocupada em determinar o comportamento da superfície, entretanto, também é desejável que a resposta seja bem estimada sobre a região de interesse.

BOX \& DRAPER $(1959,1963)$ descreveram a medida da variância de predição integrada, a qual mede a capacidade de predição de um determinado delineamento em uma região dada.

Para um determinado delineamento $D$ e para uma região $R$, a variância de prediçāo integrada é definida como

$$
\begin{aligned}
V I(D) & =\frac{N K}{\sigma^{2}} \int_{R} \operatorname{Var}(\hat{y}(\underset{\sim}{x})) d \underset{\sim}{x} \\
& =N K \int_{R}{\underset{\sim}{x}}^{\prime}\left(X^{\prime} X\right)^{-1} \underset{\sim}{x} d \underset{\sim}{X}
\end{aligned}
$$


onde $\mathrm{N}$ é o número total de pontos no delineamento e $K^{-1}=\int_{R} d \underset{\sim}{X}$ é o volume da região $R$.

Na prática, a região $R$ é uma esfera ou um cubo no espaço das variáveis independentes. O delineamento que tem a variância mínima de predição integrada é considerado o melhor delineamento de acordo com este critério. 0 fator $\mathbf{N}$ leva em conta a eficiência do delineamento. Então, um delineamento com um número menor de pontos experimentais é preferível a um delineamento maior, mais caro, mas com a mesma capacidade de predição. Ainda segundo BOX \& DRAPER (1959, 1963) a expressão (3.6.3.1) permite que se estude ó efeito do vício devido a inadequacidade da especificação do modelo na capacidade de predição de um modelo.

\subsubsection{Comparação de delineamentos}

Delineamentos na mesma região experimental podem ser comparados aos delineamentos D-ótimos e G-ótimos considerando respectivamente, a D-eficiência e G-eficiência, ATWOOD (1969).

D-eficiência mede a eficiência de um determinado delineamento para um modelo contendo p parâmetros em relação ao delineamento D-ótimo para o mesmo modelo. Assim, considere que o delineamento $\mathrm{D}^{*}$, com matriz do modelo $\mathbf{x}$ e w pontos experimentais $\mathbf{e} \mathbf{x}_{\mathbf{D}}$ a matriz do modelo para 0 delineamento D-ótimo de tamanho $\mathbb{N}_{\mathbf{D}}$. A D-eficiência para 0 delineamento $\mathrm{D}^{*}$ é definida como

$$
\operatorname{D-eficiência}\left(D^{*}\right)=\left[\frac{\left|\left(X^{\prime} X\right) / N\right|}{\left|\left(X^{\prime}{ }_{D} X_{D}\right) / N_{D}\right|}\right]^{\frac{1}{p}}
$$

a qual é a razão das variâncias generalizadas para os dois delineamentos escalados pelos seus respectivos tamanhos. 
A G-eficiência de um delineamento $D^{*}$ de tamanho $\mathrm{N}$ e com uma matrix modelo $\mathrm{x}_{G}$ é

$$
\text { G-eficiência }\left(D^{*}\right)=\frac{d_{G}}{d_{\max }}=\frac{P}{d_{\max }}
$$

onde $d_{G}=\max _{\underset{x}{x \in R}} N_{G} x_{\sim}^{\prime}\left(X_{G}^{\prime} X_{G}\right)^{-1} \underset{\sim}{X}=p$ para delineamentos G-ótimos, KIEFER \& WOLFOWITZ (1960), e $d_{\text {máx }}=\operatorname{máx}_{x \in R} \underset{\sim}{x}\left(X^{\prime} X\right)^{-1} \underset{\sim}{X}$, onde $\mathrm{R}$ é a região do delineamento em questão.

Da análise da expressão (3.6.4.2), nota-se que a G-eficiência é a razão da máxima variância de predição em alguma região dos dois delineamentos. Um delineamento G-ótimo tem uma G-eficiência igual a 1.0 , neste caso, $\mathbf{d}_{\operatorname{máx}}=\mathbf{d}_{\mathbf{G}}$, $a$ menor variância de predição máxima entre os delineamentos considerados.

\subsubsection{Rotacionalidade}

A precisão de uma predição é muito importante em problemas de superfície de resposta. Estimar a resposta ótima é de especial interesse em tais problemas. Dado que, a combinação ótima dos fatores do sistema é desconhecida, a procura por este ótimo é feita em todas as direçōes do centro do delineamento. Então, parece vantajoso estimar bem, em locais que estão na mesma distância do centro do delineamento. Assim, é interessante que a variância estimada em um determinado ponto dependa apenas da distância deste ao centro do delineamento. Então, para todos os pontos em uma hiper-esfera de raio $r$, isto é, pontos $\underset{\sim}{x}$ tais que $\Sigma x_{i}^{2}=r^{2}$, a variância de um valor previsto é uma função somente desta distância, ou seja, $\operatorname{Var} \hat{y}(x)=g(r) \sigma^{2}$. Esta propriedade é denominada de rotacionalidade ( BOX \& HUNTER, 1957).

A rotacionalidade é uma propriedade do 
delineamento de tratamentos e pode ser caracterizada através dos elementos da matriz de momentos $S=N^{-1}\left(X^{\prime} X\right)$ deste delineamento. Estes momentos são dados por

$$
\begin{gathered}
{[i]=\frac{1}{N} \sum_{u=1}^{N} x_{u i} \quad[i j]=\frac{1}{N} \sum_{u=1}^{N} x_{u i} x_{u j}} \\
{[i i j]=\frac{1}{N} \sum_{u=1}^{N} x_{u i}^{2} x_{u j} \quad[i i j j]=\frac{1}{N} \sum_{u=1}^{N} x_{u i}^{2} x_{u j}^{2}}
\end{gathered}
$$

etc. para $i, j=1,2, \ldots, k$

Momentos da forma [i] são denominados momentos de primeira ordem; [ij] são momentos de segunda ordem e assim por diante. A matriz de momentos para o modelo de primeira ordem contém somente momentos de primeira e segunda ordem. Entretanto, para o modelo de segunda ordem, momentos de quarta ordem são incluídos na matriz de momentos.

As condições necessárias e suficientes para que um delineamento, utilizado para ajustar um modelo de primeira ordem, seja rotacional são

$$
[i]=[i j]=0 \quad \text { com } i \neq j
$$

e os momentos puros de segunda ordem, [ii], tenham o mesmo valor para todo $i=1,2, \ldots, k$

No caso de um modelo de segunda ordem, um delineamento é rotacional se e somente se :

- os momentos impares envolvidos

$$
\begin{gathered}
{[i],[i j],\left[i j^{\prime}\right],} \\
\text { [iii] e[iiij], com } i \neq j=1,2, \ldots, k \quad(3.6 .5 .4)
\end{gathered}
$$

sejam todos nulos;

a) os momentos puros de segunda ordem [ii] são todos iguais, 
para todo $i=1,2, \ldots, k$, e

b) os momentos puros de quarta ordem são tais que

$$
\text { [iiii] }=3[\text { iijj] }
$$

Maiores detalhes são fornecidos por BOX \& HUNTER (1957), MYERS (1976) ou BOX \& DRAPER (1987). Note que um delineamento que é rotacional para um modelo de primeira ordem, pode não ser, necessariamente, rotacional quando um modelo de segunda ordem é utilizado. No modelo de segunda ordem, rotacionalidade não é equivalente a ortogonalidade.

Uma consequência natural da propriedade da rotacionalidade é que os contornos da variância, isto é, $\operatorname{var}\{\hat{y}(\underset{\sim}{x})\}=$ constante, são esféricas, e igual informação é obtida em qualquer direção, de mesmo raio $r$, no espaço das variáveis independentes. Delineamentos rotacionais são frequentemente utilizados em problemas de superfície de resposta. Esta importante propriedade é um aspecto desejável em qualquer delineamento. Mesmo em circunstâncias onde rotacionalidade exata não é atingida, por causa de outras restriçōes importantes, como por exemplo blocos ortogonais ou a perda de pontos experimentais durante o experimento, ainda assim, é interessante tornar o delineamento tão rotacional quanto possivel. $\overline{\mathrm{E}}$ importante saber se um determinado delineamento é rotacional ou, se ele não for, saber o quanto rotacional é o delineamento.

\subsubsection{Medindo a rotacionalidade}

A escolha de um delineamento não-rotacional ou quase-rotacional pode ser uma opção válida para o pesquisador 
em MSR.

Nas situações em que muitos pontos experimentais são necessários para a obtenção de um delineamento rotacional, o pesquisador pode escolher um delineamento menor, não-rotacional para economizar tempo e dinheiro. DRAPER \& PUKELSHEIM (1990) forneceram uma medida para determinar quão próximo um delineamento não-rotacional está desta propriedade de igual variância de predição em uma hiper-esfera. Uma notação especial foi utilizada por estes autores para a obtenção desta medida. Para o modelo de segunda ordem, eles consideraram que uma linha da matriz $\mathbf{x}$ consiste de termos

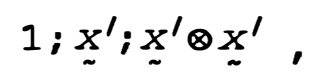

onde $\underset{z}{x}=\left(x_{1}, x_{2}, \ldots, x_{k}\right)^{\prime}$ e $\otimes$ denota o produto de Kronecker. Assim, existem $1+k+k^{2}$ termos,

$$
\begin{gathered}
1 ; x_{1}, x_{2}, \ldots, x_{k} ; x_{1}^{2}, x_{1} x_{2}, \ldots, x_{1} x_{k} ; x_{2} x_{1}, x_{2}^{2}, \ldots, x_{2} x_{k} ; \ldots \\
, x_{k} x_{1}, x_{k} x_{2}, \ldots, x_{k}^{2} .
\end{gathered}
$$

Uma desvantagem de (3.6.5.1.1) é que todos os termos de duplos produtos ocorrem duas vezes, assim a matriz X'X, correspondente é singular. Uma inversa generalizada adequada de $\mathbf{X}^{\prime} \mathbf{X}$ pode ser usada. Considerando qualquer delineamento rotacional com momentos de segunda ordem $\lambda_{2}=N^{-1} \sum_{u} x_{u i}^{2}$ e $\lambda_{4}=\sum_{u} x_{u i}^{2} x_{u j}^{2}$, pode-se escrever a matriz de momentos $v$, de ordem $\left(1+k+k^{2}\right) \times\left(1+k+k^{2}\right)$, na forma

$$
V=V_{0}+\lambda_{2}(3 k)^{\frac{1}{2}} V_{2}+\lambda_{4}[3 k(k+2)]^{\frac{1}{2}} V_{4} \quad(3 \cdot 6 \cdot 5 \cdot 1 \cdot 3)
$$

onde $v_{0}$ consiste de 1 na posição $(1,1)$ e zero no restante, $v_{2}$ consiste de $(3 \mathrm{k})^{-1 / 2}$ em cada uma das $3 \mathrm{k}$ posiçōes correspondentes aos momentos de segunda ordem em $\mathbf{v}$ e zero no restante $e v_{4}$ 
consiste de $3[3 k(k+2)]^{-1 / 2}$ nas $k$ posições correspondentes aos momentos puros de quarta ordem em $\mathbf{v}$ e zeros no restante. Nota-se que $\mathbf{v}_{0}, \boldsymbol{v}_{1}$ e $\mathbf{v}_{4}$ são simétricas e ortogonais tal que $\mathbf{v}_{\mathbf{i}} \mathbf{v}_{\mathbf{j}}=\mathbf{0}$, e também que as $\boldsymbol{v}_{\mathbf{i}}$ têm normas $\left\|V_{i}\right\|=\left[\operatorname{tr}\left(V_{i} V_{j}\right)\right]^{1 / 2}=1$. Para um delineamento arbitrário com matriz de momentos $A=N$ ${ }^{1}\left(x^{\prime} x\right)$, DRAPER et al., citado por DRAPER \& PUKELSHEIM (1990) mostraram que ponderando $A$ em todas as direções do espaço das variáveis independentes, $\underset{\sim}{X}$, obtêm-se

$$
\bar{A}=V_{0}+V_{2} \operatorname{tr}\left(A V_{2}\right)+V_{4} \operatorname{tr}\left(A V_{4}\right)
$$

onde $\bar{A}$, é denominado de componente rotacional de A. Assim a medida de rotacionalidade, $\mathbf{Q}^{*}$, a qual é baseada em $A e \bar{A}$, é definida como

$$
\begin{gathered}
Q^{*}=\left\|\bar{A}-V_{0}\right\| /\left\|A-V_{0}\right\|^{2} \\
=\left\{\operatorname{tr}\left(\bar{A}-V_{0}\right)^{2}\right\} /\left\{\operatorname{tr}\left(A-V_{0}\right)^{2}\right\}
\end{gathered}
$$

A medida $Q^{*}$, como a medida de rotacionalidade $\Phi$, apresentada por KHURI (1988), fornece uma comparação dos momentos de um delineamento não-rotacional e um delineamento rotacional de mesmo tamanho e pode ser apresentada como uma porcentagem. Se o delineamento considerado é rotacional, a distância entre as matrizes $\overline{\bar{A}}$ e $\mathbf{v}_{0}$, e $A$ e $\mathbf{v}_{0}$ são iguais $e$, $Q^{*}=100$. Um valor muito grande de $Q^{*}$ indica que os momentos do delineamento, em questão, aproximam-se muito dos delineamentos rotacionais. Neste caso, o delineamento é denominado "quase-rotacional". Um exemplo dos valores de $\mathbf{v}_{2}$ e $\mathbf{V}_{4}$ para $k=3$ é apresentado em DRAPER \& PUKELSHEIM (1990).

Um outro ponto que afeta a medida de rotacionalidade $Q^{*}$ é o da escala do delineamento examinado. Em quase todas as comparações de dois ou mais delineamentos, 
uma decisão deve ser tomada sobre a distância, da origem do delineamento, onde se deve colocar o conjunto de pontos experimentais. BOX \& HUNTER (1957) e KHURI (1988) fixaram $\lambda_{2}=1$. Neste trabalho, como em DRAPER \& PUKELSHEIM (1990), considerou-se a esfera unitária ( $k>3$ ) como a região de interesse. Assim todos os pontos experimentais foram multiplicados por um fator $f$ tal que todos os pontos ficassem dentro ou sobre a esfera de raio unitário. 0 fator $f$ escolhido neste trabalho foi o inverso da distância do centro do delineamento ao ponto axial do delineamento. Uma consequência imediata disto, é que quando pontos centrais, ou um outro, são adicionados ao delineamento, os outros pontos experimentais não precisam ser rearranjados e o valor de $\mathbf{Q}^{*}$ não se altera. Ao passo que quando se fixa $\lambda_{2}=1$, a adição de um ou mais pontos centrais requererá o rearranjo de todos os outros pontos experimentais e, mais ainda, a forma do conjunto de pontos experimentais estará distorcida axialmente.

\subsection{Delineamentos}

Os delineamentos compostos, descritos nesta seção, representam vários planos experimentais que o pesquisador pode escolher para explorar uma superfície de resposta, e são muito utilizados na prática. Recentemente, surgiu um interesse muito grande em delineamentos com um número pequeno de pontos experimentais, como uma alternativa econômica para os delineamentos de superfície de resposta com um tamanho grande. A grande maioria das investigações das propriedades destes delineamentos é feita através da Deficiência, como por exemplo, LUCAS (1974), MITCHELL (1974) e NALIMOV et al. (1970). 


\subsubsection{O delineamento fatorial $2^{k}$}

Nos delineamentos fatoriais $2^{\mathrm{k}}$, cada uma das $\mathrm{k}$ variáveis aparece em 2 níveis, geralmente em +1 e -1 . Um plano fatorial completo consiste de todas as possíveis combinaçōes dos níveis da variável. A matriz de delineamento, D, para o delineamento fatorial $2^{3}$ é a seguinte

$$
\left[\begin{array}{lll}
-1 & -1 & -1 \\
+1 & -1 & -1 \\
-1 & +1 & -1 \\
+1 & +1 & -1 \\
-1 & -1 & +1 \\
+1 & -1 & +1 \\
-1 & +1 & +1 \\
+1 & +1 & +1
\end{array}\right]
$$

A ordem destas combinaçōes deve ser aleatorizada quando o delineamento é usado. Estes delineamentos e certas fraçōes do delineamento fatorial $2^{\mathrm{k}}$ são de primeira ordem ortogonais e, portanto, rotacionais. Eles possuem, também, muitas propriedades, como por exemplo, fornecem valores mínimos para a variância de predição integrada e variância mínima generalizada, para os estimadores dos coeficientes dentre todos os outros delineamentos de primeira ordem. Os delineamentos fatoriais $2^{\mathrm{k}}$ são especialmente úteis no estágio inicial da investigação, quando não se conhece muito a respeito do sistema e o modelo ainda não foi identificado. Para $k=2$ e $k=3$ pode-se ter um padrão geométrico dos pontos experimentais. Estes são representados por pontos cujās coordenadas são os niveis +1 e -1. Assim, por exemplo, os pontos experimentais do 
delineamento $2^{3}$ estão nos vértices de um cubo.

\subsubsection{Blocos e delineamentos fatoriais fracionários $2^{k}$.}

Os delineamentos fatoriais $2^{\mathrm{k}}$ têm a importante propriedade da ortogonalidade. Por meio desta propriedade os delineamentos fatoriais podem ser divididos em $2^{\mathrm{m}}$ blocos de tamanho $2^{\mathrm{k}-\mathrm{m}}$, quase sempre sacrificando a informação das interações de ordem superior.

Efetuando o produto das três colunas do delineamento fatorial $2^{3}$ representado por (3.7.1.1), tem-se uma coluna de +1 e -1 que formam a coluna da interação de segunda ordem. Se as unidades experimentais são colocadas em dois grupos, sendo as com +1 em um bloco e as com -1 em outro, então as diferenças entre blocos serão estimadas através da interação tripla. Diz-se, então, que a interação tripla está confundida com o efeito de bloco. Por causa da propriedade da ortogonalidade do delineamento, todos os outros efeitos são estimados sem o efeito de blocos. Este delineamento tem um contraste de definição $I=123$. Aqui, tal como na notação de BOX \& DRAPER (1987), os efeitos principais são designados por números. Assim na matriz definida por (3.7.1.1) a primeira, a segunda e terceira coluna desta matriz são designadas, respectivamente, por 1, 2 e 3. Os dois blocos consistem daquelas combinações experimentais tendo, respectivamente, um número par ou impar de números em comum com 123.

Uma lista de arranjos e confundimentos de blocos úteis para delineamentos fatoriais $2^{k}$ é encontrada em BOX et al. (1978), DAVIES (1954), BOX \& DRAPER (1987). 


\subsubsection{Delineamentos fatoriais fracionários $2^{k-m}$}

Uma desvantagem dos delineamentos fatoriais é o enorme número de unidades experimentais quando $\mathrm{k}$ é grande. Como consequência, estimativas precisas dos

$\mathbf{k}$ efeitos principais,

$k(k-1) / 2$ efeitos de interações duplas,

$\mathbf{k}(\mathbf{k}-1)(\mathbf{k}-2) /(2 \times 3)$ efeitos de interações triplas e $(k(k-1)(k-2) \ldots(k-h-1)) / h$ ! efeitos de interações de ordem $h$, são obtidas. Geralmente as interações de ordem superiores podem ser desprezadas. Quando isto acontece, delineamentos fracionários, $2^{k-m}$, com um número mais econômico de pontos experimentais, podem ser utilizados.

Considere, por exemplo, o delineamento fatorial fracionário $(1 / 2) 2^{4}$ ou $2^{4-1}$. Tomando um contraste de definição $I=1234$, tem-se uma divisão das 16 combinações do experimento $2^{4}$ em dois delineamentos de 8 combinações cada um. A estrutura de confundimento é dada por

$$
\begin{gathered}
1=234 \quad 2=134 \quad 3=124 \quad 4=123 \\
12=34 \quad 13=24 \quad 14=23
\end{gathered}
$$

Assim, se as interações triplas são desprezadas, estimativas dos efeitos principais são obtidas, enquanto que as interações duplas estão confundidas aos pares. Um método alternativo de geração de um fatorial fracionário $2^{\mathrm{k}-\mathrm{m}}$ é aquele em que se inicia com um fatorial completo em $\mathrm{k}-\mathrm{m}$ fatores e $\mathrm{m}$ fatores são adicionados. Por exemplo, um delineamento fatorial fracionário $2^{4-1}$, gerado pelo contraste $4=123$, pode ser obtido impondo esta relação definidora em um fatorial $2^{3}$. Os níveis do quarto fator, são determinados pelos niveis da interação tripla do fatorial completo $2^{3}$. Delineamentos utilizados para o ajuste de modelos de segunda ordem podem ser formados através desta técnica.

os delineamentos fatoriais fracionários 
podem, por conveniência, ser classificados em tipos. Definese como a resolução $R$, de um delineamento fatorial fracionário, o comprimento da menor "palavra" na relação definidora. Por exemplo, o delineamento $2^{4-1} \mathrm{com}$ a relação definidora $I=1234$ é de resolução IV. Este delineamento é identificado pela notação $2_{I V}^{4-1} \mathrm{com}$ relação definidora $I=1234$. Em geral a resolução dos delineamentos fracionários pode ser:

III se os efeitos principais estão confundidos com as interações duplas, mas não entre si. 0 delineamento $2^{3-1}$ é de resolução III.

IV se os efeitos principais não estão confundidos com as interações duplas, mas as interações duplas estão confundidas aos pares.

$v$ se os efeitos principais e todas as interações duplas não estão confundidas entre si, entretanto as interações duplas podem estar confundidas com as interações triplas.o delineamento $2^{5-1}$ é de resolução $\mathbf{v}$.

\subsubsection{Delineamento composto central}

Os delineamentos $2^{\mathrm{k}}$ não permitem a estimação de coeficientes de um modelo de segunda ordem. Os delineamentos compostos centrais (DCC) foram desenvolvidos por BOX \& WILSON (1951) como um plano experimental que permite uma estimação eficiente dos coeficientes de segunda ordem na equação do modelo ajustado (3.1.4). O delineamento consiste de um delineamento fatorial $2^{\mathbf{k}}$ ou um delineamento fatorial fracionário, um conjunto de pontos axiais ou estrelas à distância $\alpha$, e $n_{0}$ pontos centrais. 
A matriz de delineamento de um DCC em $\mathrm{k}$ variáveis é dada por

$$
\left[\begin{array}{cccc} 
\pm 1 & \pm 1 & \ldots & \pm 1 \\
\alpha & 0 & \ldots & 0 \\
-\alpha & 0 & \ldots & 0 \\
0 & \alpha & \ldots & 0 \\
0 & -\alpha & \ldots & 0 \\
\vdots & \vdots & \ldots & \vdots \\
0 & 0 & \ldots & \alpha \\
0 & 0 & \ldots & -\alpha \\
0 & 0 & \ldots & 0
\end{array}\right]
$$

Os pontos $\pm 1 \pm 1 \ldots \pm 1$ formam a parte cubo ou fatorial, os pontos em que aparecem $\pm \alpha$, constituem a parte axial e são um aumento à parte fatorial que possibilita a estimação dos coeficientes $\beta_{i i}$, os termos quadráticos puros da equação (3.1.4). A Figura 3.7.4.1 fornece uma ilustração geométrica de um DCC de três variáveis.

Para os DCC, pode ser verificado que os momentos ímpares até de quarta ordem são zeros, isto é

$$
\begin{aligned}
& \text { [i] } \quad=0 \quad i=1,2, \ldots, k \\
& \text { [iii] }=0 \quad i=1,2, \ldots, k \\
& \text { [ij] }=0 \quad i, j=1,2, \ldots, k \quad i \neq j \\
& \text { [iij] }=0 \quad i, j=1,2, \ldots, k \quad i \neq j \\
& \text { [ijk] }=0 \quad i \neq j \neq k \\
& \text { [iiij] }=0 \quad i, j=1,2, \ldots, k \quad i \neq j \\
& \text { [iijk] }=0 \quad i \neq j \neq k
\end{aligned}
$$


FIGURA 13 - Delineamento composto central (DCC) para três fatores $(k=3)$.

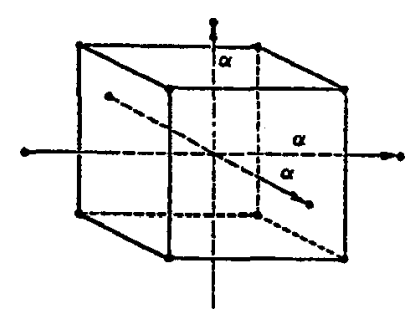

Os momentos pares, [ii], [iiii], e [iijj] $(i, j=1,2, \ldots, k ; i \neq j)$ são diferentes de zero com $[i i]=1$ pela escala definida em (3.1.2). Os momentos [iiii] e [iijj] são influenciados pela escolha do número de pontos centrais, $n_{o}$ e pelo valor de $\alpha$.

Um DCC é rotacional se $[i i i i]=3[i i j j]$ para $i, j=1,2, \ldots, k ; i \neq j$, ver equação (3.6.5.5). Se g é um fator escala escolhido tal que $[i i]=1$, então $g=$ $\left[N /\left(F+2 \alpha^{2}\right)\right]^{1 / 2}$, onde $F$ é o número de pontos na porção fatorial do delineamento, e $\mathrm{N}=\mathrm{F}+2 \mathrm{k}+\mathrm{n}_{\mathrm{o}}$ é o número total de pontos no DCC. Então, da condição de rotacionalidade, [iiii] = $3[i i j j]$, um DCC é rotacional se

$$
F g^{4}+2 \alpha^{4} g^{4}=3 F g^{4}
$$

ou, equivalentemente,

$$
\alpha=F^{1 / 4} \text {. }
$$

Um DCC é ortogonal se $[i i j j]=1$. Em termos do fator escala g, [iijj] é escrito como

$$
[i i j j]=\frac{F g^{4}}{N}=\frac{F N}{\left(F+2 \alpha^{2}\right)^{2}} .
$$

Para que $[i i j j]=1$, deve-se ter

$$
\left(F+2 \alpha^{2}\right)^{2}=\text { FN. }
$$


Resolvendo a equação para $\alpha$, conclui-se que para um DCC ser ortogonal, o valor de $\alpha$ deve ser

$$
\alpha=\left(\frac{(F N)^{\frac{1}{2}}-F}{2}\right)^{\frac{1}{2}}
$$

o valor de $\alpha$, e de $\mathbf{n}_{0}$, o número de pontos centrais são escolhidos de acordo com a propriedade do delineamento desejada. Por exemplo, considere um DCC consistindo de

(a) $n_{c}=2^{k-p} r_{c}$ pontos da parte cubo do delineamento de resolução $\mathbf{v}$ pelo menos;

(b) $n_{s}=2 k r_{s}$ pontos estrelas, e

(c) $\mathbf{n}_{\mathbf{0}}$ pontos centrais.

Então

$$
r_{c}=\frac{n_{c}}{2^{k-p}}
$$

mede o grau de repetição da parte cubo escolhida e

$$
r_{s}=\frac{n_{s}}{2 k}
$$

é o número mínimo de vezes que a parte estrela é repetida.

o DCC pode ser dividido em blocos ortogonais de tal forma que os efeitos de blocos não afetem as estimativas dos parâmetros do modelo de segunda ordem. BOX \& HUNTER (1957) apresentaram duas condiçōes que devem ser obedecidas para se obter blocos ortogonais. Suponha que existam $\mathbf{n}_{\mathrm{w}}$ combinaçōes no w-ésimo bloco. Então:

1. Cada bloco deve ser um delineamento ortogonal de primeira ordem, isto é, 


$$
\sum_{u=1}^{n_{w}} x_{i u} x_{j u}=0, i \neq j=0,1,2, \ldots, \mathrm{k} \text { para todo } \mathrm{w} .
$$

2. A fração do total da soma de quadrados de cada variável do w-ésimo bloco deve ser igual à fração do total de observações de cada bloco, isto é,

$$
\frac{\sum_{u=1}^{n_{v}} x_{i u}^{2}}{\sum_{u=1}^{N} x_{i u}}=\frac{n_{w}}{N}, \quad i=1,2, \ldots, k, \text { para todo } w .
$$

Considere a divisão do delineamento em dois blocos. Um consistindo de $\mathbf{n}_{\mathbf{c}}$ combinações da parte cubo mais $\mathbf{n}_{\mathbf{c} 0}$ pontos centrais, e a outra de $\mathbf{n}_{\mathbf{s}}$ pontos estrelas mais $\mathbf{n}_{\mathrm{so}}$ pontos centrais. Estes blocos satisfazem a primeira condição de ortogonalidade. A segunda é satisfeita se

ou seja,

$$
\frac{n_{c}}{2 r_{s} \alpha^{2}}=\frac{n_{c}+n_{c o}}{n_{s}+n_{s o}}
$$

ou alternativamente,

$$
\alpha^{2}=\frac{k \cdot n_{c}}{n_{s}}\left\{\frac{n_{s}+n_{s o}}{n_{c}+n_{c o}}\right\}=k \cdot\left\{\frac{1+\frac{n_{s o}}{n_{s}}}{1+\frac{n_{c o}}{n_{c}}}\right\} \text {, }
$$

$$
\alpha^{2}=k \cdot\left\{\frac{1+p_{s}}{1+p_{c}}\right\}
$$

onde $p_{s}=\frac{n_{s 0}}{n_{s}}$ e $p_{c}=\frac{n_{c o}}{n_{c}}$ são as proporções de pontos centrais relativos aos pontos não centrais na parte estrela e cubo, respectivamente. Mas $p_{s}$ e $p_{c}$ são tipicamente frações pequenas de mesma magnitude, tal que 0 fator $\left(1+p_{s}\right) /\left(1+p_{c}\right)$ é aproximadamente igual à unidade. Assim para um DCC ser bloco ortogonal, $\alpha$ deve ser próximo de $\mathbf{k}^{1 / 2}$, ou seja, os pontos estrelas devem ter, aproximadamente, a mesma distância do centro do delineamento aos pontos da parte cubo. 
Para um delineamento ser rotacional e bloco ortogonal ao mesmo tempo, é necessário que as equaçōes

e

$$
\alpha=\left(\frac{n_{c}}{r_{s}}\right)^{\frac{1}{4}}=\left(\frac{2 k n_{c}}{n_{s}}\right)^{\frac{1}{4}},
$$

$$
\alpha^{2}=k \cdot\left\{\frac{1+p_{s}}{1+p_{c}}\right\}
$$

devam ser satisfeitas, simultaneamente. Isto implica que

$$
\frac{I_{s}}{I_{c}}=\frac{2^{k-p}}{k^{2}}\left\{1+p_{c}\right\}^{2}
$$

Na prática, não será possível encontrar sempre a exigência exata do requerimento para bloco ortogonal e rotacionalidade, pois $\mathbf{n}_{\mathrm{c}}, \mathbf{n}_{\mathrm{s}}, \mathbf{n}_{\mathrm{c} 0}$ e $\mathbf{n}_{\mathrm{s} 0}$ devem ser números inteiros. Em particular, rotacionalidade pode ser negligenciada um pouco e o número de pontos centrais podem ser ajustados para se obter delineamentos blocos ortogonais usando a equação (3.7.4.5)

A idéia de divisão de um DCC em dois blocos pode ser estendida para vários blocos. Blocos menores podem ser utilizados se a parte fatorial ou fracionária pode ser dividida ainda mais. Dividindo igualmente os $\mathbf{n}_{\mathbf{c o}}$ pontos centrais entre estes blocos menores, o requisito para bloco ortogonal permanece satisfeito e, é claro, $\mathbf{n}_{\mathrm{co}}$ deve ser um múltiplo do número de blocos menores. Da mesma forma, se a parte estrela é repetida, então, cada repetição, com igual distribuição dos $\mathbf{n}_{\mathrm{s} 0}$ pontos centrais, pode ser feita para a obtenção de blocos ortogonais. o valor de $\alpha$ para se obter blocos ortogonais menores continua a ser o valor da equação $(3 \cdot 7 \cdot 4 \cdot 5)$. 


\subsubsection{Delineamentos compostos pequenos}

Ao se ajustar uma equação com p parâmetros, são necessários pelo menos $\mathrm{p}$ pontos experimentais. Assim, delineamentos com tamanho próximo a este número, despertam interesse dos pesquisadores nas seguintes situaçōes:

1. Quando é alto o custo das realizaçōes experimentais;

2. Quando não são necessárias: as verificaçōes das hipóteses de normalidade, a verificação do ajuste do modelo e a verificação de possíveis transformaçōes das variáveis preditoras;

3. Quando não há erro experimental, ou seja, o objetivo é aproximar localmente, através de um polinômio, uma função que pode ser calculada exatamente em qualquer combinação das variáveis independentes.

Delineamentos com um número de pontos experimentais igual ao número $\mathbf{p}$ de parâmetros a serem estimados são, também, denominados de saturados e quando o número de pontos experimentais é pouco superior a p, a denominação é quase-saturado.

\subsubsection{Delineamentos compostos de Hartley}

Quando k, o numero de fatores, é grande, a parte cubo de um DCC pode ser substituida por uma fração do fatorial $2^{k}$. Se uma fração $2^{-m}$ de um fatorial $2^{k}$ é usada na parte fatorial, então $2^{\mathrm{m}-1}$ efeitos serão sacrificados. Os $2^{\mathrm{k}-\mathrm{m}}$ efeitos do fatorial $2^{k}$ restantes são colocados em $2^{k-1}-1$ conjuntos de confundimentos (aliases), cada um contendo $2^{\mathrm{m}}$ efeitos os quais estão confundidos entre si. Com base nestes aspectos, HARTLEY (1959) provou o seguinte teorema:

Teorema.Em qualquer DCC no qual nenhum efeito principal 
é usado como uma relação definidora da fração $2^{-m}$ de um fatorial $2^{k}$, é sempre possivel estimar os seguintes parâmetros do modelo de segunda ordem, equação (3.1.4): a constante $\beta_{0} ;$ todos os parâmetros lineares $\beta_{i} \quad(i=1$, $2, \ldots, k) ;$ e todos os parâmetros dos termos quadráticos $\boldsymbol{\beta}_{i i} \quad(i=1,2, \ldots, k)$ e um parâmetro das interações duplas $\boldsymbol{\beta}_{i j}$ $(i, j=1,2, \ldots, k, i<j)$ selecionados de cada um dos conjuntos de confundimentos (aliases). Não é possível estimar mais do que um $\boldsymbol{\beta}_{i j}$ de cada conjunto de confundimento (aliases).

Então, em um DCC sob a hipotese do teorema de Hartley, o número total de parâmetros no modelo dado pela equação (3.1.4) que podem ser estimados é $2 k+1+2^{k-m}-1$, ai incluídos o $\boldsymbol{\beta}_{0}, \boldsymbol{\beta}_{i}$ para $(i=1,2, \ldots, k), \boldsymbol{\beta}_{i i}$ para $(i=1,2, \ldots, k)$ e $2^{k-m}-1$ valores de $\beta_{i j}(i, j=1,2, \ldots, k$, $i<j)$. Como o número de tratamentos dos DCC é dado por $\mathrm{N}=2^{\mathrm{k}-\mathrm{m}}$ $+2^{k}+n_{0}$, então o número de graus de liberdade para estimar a variância do erro experimental neste caso é $\mathrm{n}_{0}$, o número de repetições do ponto central.

Assim, os delineamentos apresentados por HARTLEY (1959) foram com base na observação de que o fatorial fracionário escolhido não precisa fornecer estimadores não correlacionadas com todos os efeitos principais e interações duplas. A exigência é que os estimadores de interações duplas não sejam correlacionadas entre si.

HARTLEY (1959) propôs o delineamento apresentado na Tabela 1 do Apêndice, para quatro fatores. A parte cubo é baseada no fatorial fracionário $(1 / 2) 2^{4}$, gerado pela relação definidora $1=23$. Os sete conjuntos de efeitos confundidos são
$1=23$,
$2=13$,
$3=12$,
$4=1234$. 


$$
14=(234), 24=(134), 34=(124) .
$$

Os efeitos entre parênteses são interações triplas ou superiores e não representam qualquer coeficiente no modelo representado pela equação (3.1.4). Percebe-se que todas as interações de dois fatores ocorrem em diferentes conjuntos de confundimento tal que todos os seis $\beta_{i j}$ podem ser estimados juntamente com todos os $\beta_{i}$, todos os $\beta_{i i}$ e $\beta_{0}$, quando esta fração sugerida por HARTLEY (1959) para quatro fatores é usada.

Para $\mathrm{k}=5$, o mesmo autor utilizou o fatorial fracionário recomendado por DAVIES (1954), gerado pelo contraste de definição $I=12345$. Esta fração tem todos os efeitos principais e interações duplas em diferentes conjuntos de confundimento tal que todos os $\beta_{i j}$ são estimados, juntamente com os $\beta_{i}, \beta_{i i}$ e $\beta_{0}$.

A fração $\frac{1}{2} 2^{6}$ foi utilizada por HARTLEY (1959) para seis fatores, a qual é gerada pelo contraste de definição

$$
1=23,4=56,123=456 .
$$

Limitando-se aos efeitos principais $e$ aos efeitos de interação dupla, os 15 conjuntos de confundimento são

$$
i=23,2=13,3=12,4=56,5=46 \text { e } 6=45 \text {, }
$$

enquanto que as nove interaçōes duplas restantes estão confundidas somente com outros efeitos de ordem superior.

No caso de sete fatores, a fração $\frac{1}{4} 2^{7}$ gerada pelo contraste de definição $123=456$, a qual permite estimar todos os $\boldsymbol{\beta}_{i j}$, juntamente com os $\boldsymbol{\beta}_{i} \boldsymbol{\beta}_{i i}$ e $\boldsymbol{\beta}_{0}$. Os delineamentos de HARTLEY (1959) são apresentados nas Tabelas 
$1,2,3$ e 4 do Apêndice.

\subsubsection{Delineamentos compostos de Westlake}

Os delineamentos compostos pequenos de WESTLAKE (1965) são baseados em frações irregulares do sistema fatorial $\mathbf{2}^{\mathbf{k}}$, colocados na parte fatorial dos DCC ao contrário dos fatoriais completos ou frações regulares empregadas por BOX \& WILSON (1951) e HARTLEY (1959). Este sistema de construção é bastante engenhoso.

WESTLAKE (1965) forneceu delineamentos para $k=5$, baseado no fatorial fracionário $\frac{3}{8} 2^{5}$, com contraste de definição $2=1234$ e $5=-1234$. Para $k=7$, este autor empregou a fração $\frac{13}{64} 2^{7}$ gerada pelo contraste de definição

$$
23=34=35=16=17=1
$$

Os delineamentos sugeridos por WESTLAKE (1965) e estudados neste trabalho, encontram-se descritos nas Tabelas 11 e 12 do Apêndice.

\subsubsection{Delineamentos compostos de Draper-Lin}

Uma proposta alternativa para se obter delineamentos compostos pequenos foi usada por DRAPER (1985). Este autor utilizou colunas dos delineamentos de PLACKETT \& BURMAN (1946) ao invés dos fatoriais fracionários regulares ou irregulares na parte cubo dos DCC. Uma vantagem de se utilizar os delineamentos de PLACKETT \& BURMAN é a facilidade da construção destes delineamentos. Mais precisamente, podese: 
(a) usar para a parte cubo do delineamento, $\mathbf{k}$ colunas do delineamento de PLACKETT \& BURMAN, e

(b) remover pontos experimentais quando houver repetiçōes, a fim de se reduzir o número total de pontos experimentais requeridos.

Assim, DRAPER (1985) obteve delineamentos compostos pequenos para $k=5,7$ e 9. Delineamentos compostos pequenos para outros valores de $k$, também, foram obtidos por DRAPER \& LIN (1990). A técnica utilizada consistiu também, na escolha de $\mathrm{k}$ colunas de um delineamento de tamanho $\mathbf{n}_{\mathrm{pb}}$ de PLACKETT e BURMAN. Uma questão de interesse que surge é quantos delineamentos existem para determinados valores de $\mathbf{k}$ e $\mathbf{n}_{\mathrm{pb}}$. Para distinguir delineamentos que são intrinsicamente diferentes e delineamentos que são obtidos de outras formas, como mudança dos sinais nas colunas, rearranjo nas linhas, e renomeação das variáveis, DRAPER \& LIN (1990) caracterizaram os delineamentos através do padrão de sinais, do padrão da imagem-espelho e através do valor da estatística ótima relativa $D=\left|\mathbf{X}^{\prime} \mathbf{x}\right| / \mathbf{n}^{\mathbf{p}}$, a qual descreve a informação por ponto do delineamento.

Como exemplo, para $k=4$, o número mínimo de pontos experimentais possíveis na parte fatorial é 7 , e então um delineamento de PLACKETT \& BURMAN com 8 pontos é considerado. As colunas 1,2,3,6 forneceram o maior valor da da estatística ótima relativa $D$. Este delineamento é um fatorial fracionário $2_{I I I^{*}}^{4-1}$, e é equivalente ao delineamento encontrado por HARTLEY (1959) para 4 fatores.

No caso de cinco fatores $(k=5)$, foram utilizadas cinco colunas de um delineamento de PLACKETT \& BURMAN de 12 pontos, dado que 11 é o número mínimo de pontos possíveis requeridos pela parte cubo do delineamento composto para cinco fatores. As colunas 1,2,3,4,5 forneceram o maior valor da estatística ótima relativa $D$, enquanto que as 
colunas $1,2,3,5,8$ produzem um par de pontos experimentais repetidos, que depois da remoção de um deles, tem-se um delineamento com 11 pontos experimentais na parte cubo do delineamento, fornecendo um delineamento saturado.

Para seis fatores $(k=6)$ um delineamento pequeno, saturado, é automaticamente obtido quando seis colunas apropriadas de um delineamento de 16 pontos de PLACKETT \& BURMAN é utilizado. As colunas $1,2,3,4,5,14$ foram selecionadas pela estatística ótima relativa $D$. Este delineamento é equivalente ao delineamento de HARTLEY $2_{I I I^{*}}^{6-2}$. Para estimar os 36 coeficientes do modelo de segunda ordem para sete fatores, são necessários, além do 14 pontos da parte axial, um número mínimo de 22 pontos experimentais na parte cubo. O menor delineamento de PLACKETT \& BURMAN que pode ser utilizado, então, é o que contém 24 pontos experimentais. Para este $n_{p b}=24$, as colunas $1,2,3,5,6,7,9$ forneceram a maior estatística ótima relativa D. As colunas $1,2,5,6,7,9,10$ produziram dois pares de pontos experimentais repetidos, o que permitiu a eliminação de 2 pontos experimentais, um de cada par repetido. Este delineamento saturado com 22 pontos experimentais na parte cubo não é somente menor que o delineamento de HARTLEY com 32 pontos experimentais, mas é menor, também, que o delineamento de WESTLAKE com 26 pontos experimentais. Os delineamentos de Draper-Lin estudados neste trabalho estão descritos nas Tabelas 5 a 10 do Apêndice.

\subsubsection{Delineamentos compostos de Lucas}

LUCAS (1974) definiu os menores delineamentos compostos simétricos nos pontos estrelas como consistindo de: 
(i) um ponto central;

(ii) pontos estrelas, um em $+\alpha$ e outro em $-\alpha$ para cada fator;

(iii) pontos tendo a estrutura $(0, \ldots, 0,1,0, \ldots 0,1,0, \ldots, 0)$

o ponto do item (iii) é um vetor de dimensão $k \times 1$ tendo 1 na i-ésima e na j-ésima posições e zeros nas outras. Este ponto permite a estimação dos coeficientes da interação dupla, $\mathbf{x}_{\mathbf{i}} \mathbf{x}_{\mathbf{j}}$ do modelo de segunda ordem. É necessário um ponto com esta estrutura para cada interação dupla colocada no modelo de segunda ordem. Se todos os coeficientes são estimados, existirá $\left(\begin{array}{l}k \\ 2\end{array}\right)$ pontos deste tipo.

Para estes delineamentos, têm-se

$$
\left|X^{\prime} X\right|=2^{2 k} \alpha^{6 k}
$$

o qual é uma função crescente de $\alpha$. Estes delineamentos estão listados nas Tabelas 13 a 16 do Apêndice.

3.8. Capacidade de reprodução do ponto ótimo e número médio de inversões.

3.8.1. O método utilizado

A avaliação da capacidade de reprodução do ponto ótimo pelos delineamentos de superfície de resposta possuindo 4 a 7 fatores, descritos na seção 3.7 .5 e apresentados nas Tabelas do Apêndice, foi através da simulação computacional de dados. o algorítmo de simulação foi fundamentado no trabalho de BOX \& MULLER (1958). Para tanto, as seguintes situações foram propostas: 


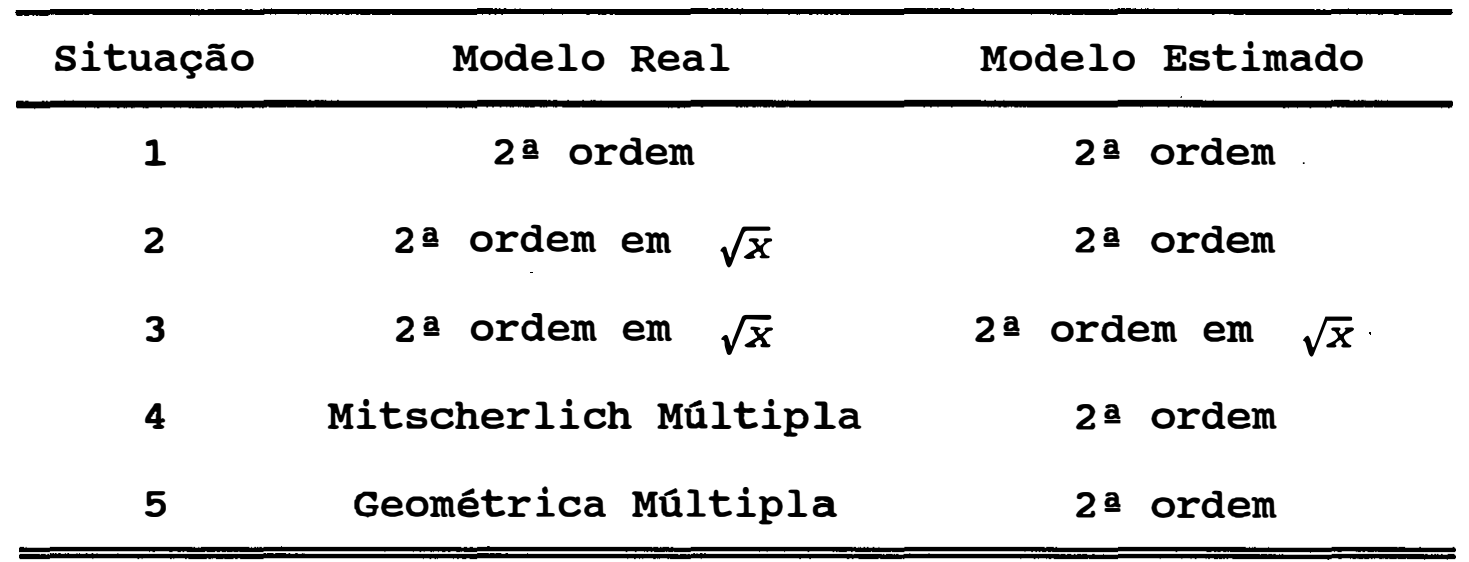

são 4 modelos reais para cada situação, um para cada número de fatores. Todos os delineamentos, com o mesmo número de fator, foram testados pelo mesmo modelo. Ao todo foram construídos 16 modelos reais, os da situações 2 e 3 são iguais. De cada modelo real e para cada valor de $\sigma$, mil (1000) vetores de respostas foram simuladas. Em seguida, para cada situação, ajustou-se os modelos polinomiais de segunda ordem propostos para cada situação e a natureza do ponto crítico classificada (máximo, mínimo, sela ou indeterminação) de acordo com a metodologia descrita na seção 3.5.1, e as ocorrências expressas em porcentagens sobre as mil (1000) simulações. Uma outra medida considerada, para as mil (1000) simulações foi o número médio de inversōes (NMinv) dos eixos dos fatores. Para as situações 1,2 e 3 , os modelos reais foram construídos com máximo dentro da região experimental que, neste trabalho, foi previamente definida como

$$
\begin{array}{r}
R=\left\{\left(x_{1}, x_{2}, \ldots, x_{k}\right) \in \mathbb{R}^{k} / 0 \leq x_{i} \leq 5,\right. \\
i=1,2, \ldots, k \text { e } k=4,5,6,7\}
\end{array}
$$

Portanto a medida NMinv fornece, para cada delineamento, 0 número médio de eixos com curvatura invertidos em um conjunto de mil (1000) simulações. Será preferível um delineamento com um NMinv pequeno quando as porcentagens de ponto de sela ou de ponto de mínimo forem altas. 
Todos os delineamentos estudados neste trabalho são compostos, com os níveis de cada fator $x_{i}$ assumindo valores

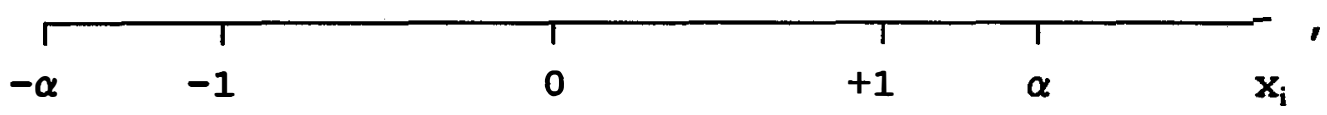

os quais são reescalonados na região $R$ definida acima. Duas formas da região $\mathrm{R}$ foram çonsideradas: a região esférica (E), a qual é definida por $\sum_{i=1}^{k} x_{i}^{2} \leq k$; e a região cuboidal (C), que consiste de todos os ${ }^{i}$ pontos sobre ou dentro do hipercubo $k$-dimensional, tal que $0 \preceq x_{i} \preceq 5, i=1,2, \ldots, k$ e k=4, 5,6 e 7 .

\subsubsection{Obtenção de modelos de segunda ordem com 4 a 7 fatores.}

SANCHES (1991) apresenta uma técnica para a construção de modelos matemáticos de superfície de respostas em condiçōes pré-estabelecidas quanto à natureza e localização do ponto crítico para um número qualquer de variáveis (fatores). Esta técnica foi aqui utilizada para a obtenção de modelos polinomiais de segunda ordem e polinomial com raiz quadrada (situações 1,2 e 3). Tal como em SANCHES (1986) a construção dos modelos nas situaçōes 1,2 e 3 foi tomada com média de, 3500 aproximadamente, um valor de $\sigma=300$ para o desvio padrão do erro de cada observação e, também, para a localização do ponto ótimo, o que dá um coeficiente de variação (c.v.) em torno de $8,5 \%$ Dois outros valores de $\sigma$ também foram considerados, $\sigma=100$ e 250 que correspondem a c.v. de $2,5 \%$ e $7 \%$, respectivamente, com a finalidade de se perceber alguma tendência . 
Esta técnica de se obter um modelo polinomial de segunda ordem, consiste em estabelecer valores para $Y_{0}, X^{*}, M$ e $\Omega$ na equação (3.5.1.7).

A extensão desta técnica para a obtenção de outros modelos, como por exemplo, modelo com expoentes fracionários, modelo exponencial, modelo logaritmico é imediato (ver SANCHES (1991)).

Para os modelos reais das situações 1,2 e 3 considerou-se uma resposta máxima de 5000 no ponto $x^{*}=\left(x_{01}, x_{02}, \ldots, x_{0 k}\right)^{\prime}=(4,4, \ldots, 4)^{\prime}$ com $\mathrm{k}=4,5,6,7$. Este ponto encontra-se dentro da região experimental préestabelecida $[0,5]$, para cada fator, e suas coordenadas estão próximas dos níveis mais altos. A matriz $\mathbf{M}$ ortogonal utilizada foi a matriz de Helmert (SEARLE 1982) e cuja forma é dada por

$$
H_{(k)}=\left[\begin{array}{cc}
h^{\prime} \\
1 & k \\
---- \\
H^{*} \\
k-1 k
\end{array}\right]
$$

onde, $h$ é um vetor com $k$ componentes iguais a $\frac{1}{\sqrt{k}}$; H é uma matriz de k-1 linhas e $\mathrm{k}$ colunas, onde a $\mathrm{r}$-ésima linha tem a forma

$$
\left[\frac{1}{\sqrt{r(r+1)}} p^{\prime},-\frac{r}{\sqrt{r(r+1)}}, \phi\right]
$$

sendo:

p um vetor com $r$ elementos iguais a 1;

ф um vetor nulo com $\mathrm{k}-\mathrm{r}-1$ elementos. 


\section{Situação 1:}

De uma maneira geral, os 4 modelos dessa situação têm:

$$
\begin{aligned}
\Omega= & \operatorname{diag}\{-100,-100, \ldots,-100\} \text { e } M=H_{k} \text { com } k=4, \ldots, 7, \\
& -2 \underline{\sim}^{* \prime} Q \underset{\sim}{x}=800 x_{1}+800 x_{2}+\ldots+800 x_{k} \text { e } \\
& x_{\sim}^{\prime} Q \underset{\sim}{x}=-100 x_{1}^{2}-100 x_{2}^{2}-\ldots-100 x_{k}^{2} \text { e } \hat{y}_{0}=5000 .
\end{aligned}
$$

Portanto, para 4 fatores têm-se

$$
\begin{gathered}
{\underset{\sim}{*}}_{\sim \prime}^{* \prime} Q{\underset{\sim}{*}}^{*}=-6400, \text { logo } \\
y=-1400+800 x_{1}+800 x_{2}+800 x_{3}+800 x_{4}-100 x_{1}^{2} \\
-100 x_{2}^{2}-100 x_{3}^{2}-100 x_{4}^{2} .
\end{gathered}
$$

Para 5 fatores, $x_{\sim}^{* \prime} Q x_{\sim}^{*}=-8000$, o que dá

$$
\begin{aligned}
y= & -3000+800 x_{1}+800 x_{2}+800 x_{3}+800 x_{4}+800 x_{5} \\
& -100 x_{1}^{2}-100 x_{2}^{2}-100 x_{3}^{2}-100 x_{4}^{2}-100 x_{5}^{2},
\end{aligned}
$$

e para 6 fatores, $x_{\sim}^{* \prime} Q x_{\sim}^{*}=-9600$, assim

$$
\begin{aligned}
y= & -4600+800 x_{1}+800 x_{2}+800 x_{3}+800 x_{4}+800 x_{5}+800 x_{6} \\
& -100 x_{1}^{2}-100 x_{2}^{2}-100 x_{3}^{2}-100 x_{4}^{2}-100 x_{5}^{2}-100 x_{6}^{2}
\end{aligned}
$$

e, finalmente, para 7 fatores, $\underset{\sim}{x^{* \prime}} Q x_{\sim}^{*}=-11200$, 0 que implica no modelo

$$
\begin{aligned}
y= & -6200+800 x_{1}+800 x_{2}+800 x_{3}+800 x_{4}+800 x_{5}+800 x_{6}+800 x_{7} \\
& -100 x_{1}^{2}-100 x_{2}^{2}-100 x_{3}^{2}-100 x_{4}^{2}-100 x_{5}^{2}-100 x_{6}^{2}-100 x_{7}^{2} .
\end{aligned}
$$

Os valores negativos dos interceptos dos modelos nesta situação são devidos aos altos valores em modulo dos elementos da matriz $\Omega$, os quais refletem a curvatura do modelo polinomial de segunda ordem. 


\section{Situaçōes 2 e 3:}

Ainda, segundo as técnicas desenvolvidas por SANCHES (1991), e considerando-se as hipóteses préestabelecidas no início desta seção, a determinação do modelo polinomial de segunda ordem em $\sqrt{x_{i}}$ descrito pela equação (3.1.5), requer, primeiro, a construção de um modelo polinomial de 2 a ordem, em $x_{i}$, com máximo no ponto $x_{0}=(2,2, \ldots, 2)^{\prime}$. A partir desse modelo, e considerando a transformação $\phi_{i}\left(x_{i}\right)=\sqrt{x_{i}}, i=1,2, \ldots, k$ e substituindo $x_{i}$ por $\phi_{i}\left(x_{i}\right), i=1,2, \ldots, k$, obtem-se o modelo polinomial de 2 a ordem em $\sqrt{x_{i}}$, com máximo em $x_{0}=(4,4, \ldots, 4)^{\prime}$.

Assim sendo, dado

$$
\begin{aligned}
& \Omega=\operatorname{diag}\{-100,-100, \ldots,-100\} \text { e } M=H_{(k)} \quad \mathrm{e} \\
& \underset{\sim}{x_{0}}=(2,2, \ldots, 2)^{\prime} \text {, têm-se então que } \\
& -2{\underset{\sim}{x}}^{* \prime} Q \underset{\sim}{x}=400 x_{1}+400 x_{2}+\ldots+400 x_{4} \text {, } \\
& \underline{x}^{\prime} Q \underset{\sim}{x}=-100 x_{1}^{2}-100 x_{2}^{2}-\ldots-100 x_{4}^{2} \text { e } \hat{y_{0}}=5000
\end{aligned}
$$

Portanto, para 4 fatores, $x_{\sim}^{* \prime} Q x_{\sim}^{*}=-800$, e o modelo fica:

$$
y=4200+400 \sqrt{X_{1}}+400 \sqrt{X_{2}}+400 \sqrt{X_{3}}+400 \sqrt{X_{4}}-100 X_{1}-100 X_{2}-100 X_{3}-100 X_{4} \text {. }
$$

Para 5 fatores, ${\underset{\sim}{x}}^{* \prime} Q{\underset{\sim}{*}}^{*}=-1000$, resultando o modelo

$$
\begin{gathered}
y=4000+400 \sqrt{X_{1}}+400 \sqrt{X_{2}}+400 \sqrt{X_{3}}+400 \sqrt{X_{4}}+400 \sqrt{X_{5}} \\
-100 X_{1}-100 X_{2}-100 X_{3}-100 X_{4}-100 X_{5} .
\end{gathered}
$$

No caso de 6 fatores, $\underset{\sim}{x^{* \prime}} Q \underset{\sim}{x^{*}}=-1200$, o que dá o modelo real 


$$
\begin{gathered}
y=3800+400 \sqrt{X_{1}}+400 \sqrt{X_{2}}+400 \sqrt{X_{3}}+400 \sqrt{X_{4}}+400 \sqrt{X_{5}}+400 \sqrt{X_{6}} \\
-100 X_{1}-100 X_{2}-100 X_{3}-100 X_{4}-100 X_{5}-100 X_{6} .
\end{gathered}
$$

E para 7 fatores, $\underset{\sim}{x^{* \prime}} Q{\underset{\sim}{*}}^{*}=-1400$, o que implica no modelo

$$
\begin{gathered}
y=3600+400 \sqrt{X_{1}}+400 \sqrt{X_{2}}+400 \sqrt{X_{3}}+400 \sqrt{X_{4}}+400 \sqrt{X_{5}}+400 \sqrt{X_{6}}+400 \sqrt{X_{7}}- \\
100 X_{1}-100 X_{2}-100 X_{3}-100 X_{4}-100 X_{5}-100 X_{6}-100 X_{7}
\end{gathered}
$$

\section{Situação 4:}

A forma exponencial da equação de Mitscherlich para uma variável é

$$
y=A\left(1-e^{-c x}\right)
$$

sendo que y a resposta média, A é a resposta máxima quando $\mathbf{x}$ é aumentado até 0 seu limite, $\mathbf{x}$ é o fator de entrada no experimento e c é a constante representando o efeito do fator $x$ na resposta. Quando $x$ tende a infinito, $y$ tem uma assíntota em $A$.

Na prática não é o valor absoluto de $\mathrm{x}$ que é medido, mas de $x_{1}$, que é o excesso de $x$ ao lado de algum valor de b já presente e desconhecido. Então, a equação pode ser reescrita como

$$
y=A\left[1-e^{-c\left(x_{1}+b\right)}\right]
$$

Uma generalização da função de Mitscherlich em $\mathrm{k}$ variáveis é dada por

$$
y=A\left(1-e^{-c_{1}\left(x_{1}+b_{1}\right)}\right)\left(1-e^{-c_{2}\left(x_{2}+b_{2}\right)}\right) \ldots\left(1-e^{-c_{k}\left(x_{k}+b_{k}\right)}\right)
$$

onde A é a resposta máxima quando $x_{i}, i=1, \ldots, k$ é aumentado até o seu limite, $x_{i}, i=1, \ldots, k$ são os fatores independentes 
e $c_{i}, i=1, \ldots, k$ são as constantes representando os efeitos dos fatores $x_{i}$ na resposta $e b_{i}$ são os valores do fator $x_{i} j a ́$ presentes quando $x_{i}=0$.

Assim, os modelos reais para geração de dados, na situação 4 , ficam:

$$
\begin{gathered}
y=5000\left[1-e^{-0,757\left(x_{1}+1,2\right)}\right]\left[1-e^{-0,555\left(x_{2}+1,5\right)}\right] \\
{\left[1-e^{-0,665\left(x_{3}+1,5\right)}\right]\left[1-e^{-0,800\left(x_{4}+2,0\right)}\right],} \\
y=5000\left[1-e^{-0,567\left(x_{1}+1,5\right)}\right]\left[1-e^{-0,658\left(x_{2}+1,8\right)}\right]\left[1-e^{-0,750\left(x_{3}+1,0\right)}\right] \\
{\left[1-e^{-0,567\left(x_{4}+2,0\right)}\right]\left[1-e^{-0,700\left(x_{5}+1,8\right)}\right],} \\
y=5000\left[1-e^{-0,540\left(x_{1}+1,4\right)}\right]\left[1-e^{-0,650\left(x_{2}+1,2\right)}\right] \\
{\left[1-e^{-0,750\left(x_{3}+1,0\right)}\right]\left[1-e^{-0,807\left(x_{4}+1,7\right)}\right] e} \\
{\left[1-e^{-0,450\left(x_{5}+2,0\right)}\right]\left[1-e^{-0,599\left(x_{6}+1,5\right)}\right]} \\
y=5000\left[1-e^{-0,678\left(x_{1}+1,2\right)}\right]\left[1-e^{-0,722\left(x_{2}+1,7\right)}\right] \\
{\left[1-e^{-0,822\left(x_{3}+2,0\right)}\right]\left[1-e^{-0,433\left(x_{4}+1,8\right)}\right]} \\
{\left[1-e^{-0,940\left(x_{5}+1,0\right)}\right]\left[1-e^{-0,654\left(x_{6}+1,1\right)}\right]} \\
{\left[1-e^{-0,576\left(x_{7}+1,9\right)}\right]}
\end{gathered}
$$

para $k=4,5$, 6 e 7 fatores, respectivamente. A magnitude dos valores dos coeficientes $c_{i} e b_{i}$ foram baseados nos exemplos relatados em HEXEM \& HEADY (1978).

\section{Situação 5:}

A função potência para $\mathrm{k}$ variáveis é dada por

$$
y=\beta_{0} x_{1}^{\beta_{1}} x_{2}^{\beta_{2}} \ldots x_{k}^{\beta_{k}}
$$

Os fatores $x_{1}, x_{2}, \ldots, x_{k}$ são limitados no sentido de que se todas os $x_{i}$ são zero, $y$ também será igual a zero. Um $y$ máximo não é definido para a função potência, 
consequentemente esta função não é decrescente. A variável resposta $y$ cresce indefinidamente.

os modelos reais propostos para geração de dados desta situaçâo são

$$
\begin{gathered}
\text { ' } y=1032,71 x_{1}^{0,157} x_{2}^{0,092} x_{3}^{0,447} x_{4}^{0,254}, \\
5 \quad y=1773,51 x_{1}^{0,202} x_{2}^{0,075} x_{3}^{0,126} x_{4}^{0,066} x_{5}^{0,175}, \\
6 y=1636,38 x_{1}^{0,25} x_{2}^{0,076} x_{3}^{0,097} x_{4}^{0,165} x_{5}^{0,047} x_{6}^{0,059} e \\
\Rightarrow y=849,97 x_{1}^{0,325} x_{2}^{0,126} x_{3}^{0,045} x_{4}^{0,288} x_{5}^{0,099} x_{6}^{0,133} x_{7}^{0,085},
\end{gathered}
$$

para $k=4,5,6$ e 7 fatores respectivamente. A magnitude dos valores de $\beta_{i}(i=1,2, \ldots, k)$ foram baseados em exemplos descritos em HEXEM \& HEADY (1978), e seus valores foram fixados de tal forma que $y=5000$ quando todos os $x_{i}$ são iguais a 5 na região experimental $R$ definida em (3.8.1). 


\section{RESULtADOS E DIscUSSĀO}

Os resultados para os critérios ótimos, para a porcentagem de reprodução do ponto ótimo e a robustez quanto a má especificação do modelo dos delineamentos compostos pequenos estudados neste trabalho, são apresentados de acordo com as situaçōes estabelecidas na seção 3.8.1., enquanto que os resultados da medida da rotacionalidade e das possibilidades de arranjos em blocos ortogonais independem destas situaçōes.

\subsection{Resultados dos critérios ótimos}

A Tabela 1 apresenta os valores dos critérios A, $\mathrm{D}$ e E-ótimos dos delineamentos $\operatorname{com} \mathrm{k}=4,5,6$ e 7 fatores, na situação 1, para a região esférica.

Caso $k=4$. Os delineamentos de Hartley e Draper-Lin apresentam os mesmos resultados para os critérios A-, D- e E-ótimos. Isto confirma as observaçōes de DRAPER \& LIN (1990) de que, quando $n_{p b}$, o número de colunas do delineamento de Plackettt e Burman, é uma potência de 2 , os delineamentos de Hartley e Draper-Lin são equivalentes, ou seja, os delineamentos de Plackett e Burman são equivalentes aos delineamentos fatoriais fracionários $2^{\mathrm{k}-\mathrm{m}}$, quanto aos critérios ótimos.

Os valores dos critérios A, D e E-ótimos para os delineamentos de Hartley e Draper-Lin são inferiores aos do delineamento de Lucas, os quais têm um ponto a mais do que 
o delineamento de Lucas, que é saturado.

Caso $k=5$. 0 delineamento de Hartley apresenta o menor valor para o critério D-ótimo, seguido pelos delineamentos de Westlake, Draper-Lin saturado, DraperLin 22 pontos e por último pelo delineamento de Lucas. Com relação ao valor do critério A-ótimo, o delineamento de Hartley apresenta, também, os menores valores, seguido pelos delineamentos de Draper-Lin 22 pontos, Draper-Lin saturado, Westlake e por último com o maior valor, o delineamento de Lucas. Os delineamentos mostram uma equivalência com respeito ao critério E-ótimo.

Caso $k=6$. Como era de se esperar, verificase novamente a equivalência entre os delineamentos de Hartley e o de Draper-Lin. Estes delineamentos apresentam os menores valores para o critério D-ótimo. O delineamento de Lucas apresenta valores para os critérios A e E-ótimos inferiores aos apresentados pelos delineamentos de Hartley e Draper-Lin.

Caso $k=7$. Novamente, 0 delineamento de Hartley apresenta os menores valores para os critérios A, D e E-ótimos. Em outro grupo de delineamentos, com valores crescentes para o critério D-ótimo, têm-se os delineamentos de Draper-Lin 38 pontos, Westlake e Draper-Lin saturado e o de Lucas. Para os valores do critério E-ótimo, observa-se uma equivalência entre estes delineamentos.

A Tabela 2 apresenta os valores dos critérios ótimos dos delineamentos, para a situação 1 , na região cuboidal. De uma maneira geral, verifica-se que as tendências observadas na região esférica, se mantiveram.

Caso $k=4$. A equivalência entre os delineamentos de Hartley e Draper-Lin, observada na região esférica, voltou a ser confirmada. Estes delineamentos apresentam os menores valores para os critérios A, D e E- 
ótimos do que os do delineamento de Lucas.

Caso $k=5$. Observa-se que o delineamento de Hartley apresenta os menores valores para os critérios A e Dótimos. Em outro grupo de delineamentos com valores crescentes do critério D-ótimo, tem-se os delineamentos de Westlake, Draper-Lin 22 pontos e Draper-Lin saturado e o de Lucas. Com relação ao critério A-ótimo, os delineamentos de Draper-Lin 22 pontos, Draper-Lin saturado e Westlake apresentam valores equivalentes. 0 delineamento de Lucas apresenta o maior valor para o critério A-ótimo e o menor valor do critério E-ótimo, seguido pelos delineamentos de Draper-Lin saturado e Draper-Lin 22 pontos e Westlake. 0 maior valor do critério E-ótimo é apresentado pelo delineamento de Hartley.

Caso $k=6 . A$ equivalência entre os delineamentos de Hartley e Draper-Lin é mantida, os quais apresentam os menores valores para os critérios A e D-ótimos. o delineamento de Lucas apresenta o menor valor para o critério E-ótimo.

Caso $k=7$. Para esta dimensão, o delineamento de Hartley apresenta os menores valores para os critérios A, e D-ótimos seguidos pelos delineamentos de Westlake, DraperLin 38 pontos, Draper-Lin saturado e por último o delineamento de Lucas. Já com relação ao critério E-ótimo, o delineamento de Lucas apresenta o menor valor, seguido pelos delineamentos de Draper-Lin 38 pontos, Draper-Lin saturado, Hartley e Westlake.

Portanto pelos resultados apresentados nas Tabelas 1 e 2 o delineamento de Hartley destaca-se pelos menores valores dos critérios A, D e E-ótimos em todas as dimensōes e nas duas regiōes experimentais. Assim, o delineamento composto pequeno de Hartley com 16 pontos 
TABELA 1 - Valores para os critérios A, D e E-ótimos dos delineamentos compostos na região esférica e na situação 1 .

\begin{tabular}{|c|c|c|c|c|c|c|}
\hline \multirow[b]{2}{*}{ Del. } & \multirow[b]{2}{*}{ Dim. } & \multirow[b]{2}{*}{$\mathbf{P}$} & \multirow[b]{2}{*}{$\mathbf{N}$} & \multicolumn{3}{|c|}{ CRITÉRIOS ÓTIMOS** } \\
\hline & & & & A & D & $\mathbf{E}$ \\
\hline & 4 & 15 & & & & \\
\hline Hartley & & & 16 & $0,34 \mathrm{E} 03$ & $0,14 \mathrm{E} 16$ & $0,73 \mathrm{E} 00$ \\
\hline Draper-Iin & & & 16 & $0,34 \mathrm{E03}$ & $0,14 \mathrm{E} 16$ & $0,73 \mathrm{EOO}$ \\
\hline Lucas* & & & 15 & $0,11 \mathrm{E} 04$ & $0,20 \mathrm{E} 21$ & $0,80 \mathrm{EOO}$ \\
\hline & 5 & 21 & & & & \\
\hline Hartley & & & 26 & $0,76 \mathrm{E03}$ & $0,21 \mathrm{E} 25$ & $0,13 \mathrm{E} 01$ \\
\hline Draper-Lin* & & & 21 & $0,95 \mathrm{E} 03$ & $0,15 \mathrm{E} 26$ & $0,13 \mathrm{E} 01$ \\
\hline Draper-Iin & & & 22 & $0,89 \mathrm{E03}$ & $0,25 \mathrm{E} 26$ & $0,13 \mathrm{E} 01$ \\
\hline Lucas* & & & 21 & $0,35 \mathrm{E} 04$ & $0,56 \mathrm{E} 35$ & $0,12 \mathrm{EO} 1$ \\
\hline Westlake & & & 22 & $0,96 \mathrm{E} 03$ & $0,10 \mathrm{E} 26$ & $0,13 \mathrm{E01}$ \\
\hline & 6 & 28 & & & & \\
\hline Hartley* & & & 28 & $0,35 \mathrm{E} 01$ & $0,20 \mathrm{E} 37$ & $0,13 \mathrm{E01}$ \\
\hline Draper-Iin* & & & 28 & $0,35 \mathrm{E01}$ & $0,20 \mathrm{E} 37$ & $0,13 \mathrm{E} 01$ \\
\hline Lucas* & & & 28 & $0,22 \mathrm{EO} 1$ & $0,35 \mathrm{E} 55$ & $0,11 \mathrm{E} 01$ \\
\hline & 7 & 36 & & & & \\
\hline Hartley & & & 46 & $0,27 \mathrm{E04}$ & $0,63 \mathrm{E} 55$ & $0,12 \mathrm{E} 01$ \\
\hline Draper-Iin & & & 38 & $0,84 \mathrm{EO} 4$ & $0,13 \mathrm{E} 56$ & $0,12 \mathrm{E} 01$ \\
\hline Draper-Lin* & & & 36 & $0,19 \mathrm{E} 05$ & $0,65 \mathrm{E} 56$ & $0,12 \mathrm{E} 01$ \\
\hline Lucas* & & & 36 & $0,26 \mathrm{E} 05$ & $0,38 \mathrm{E} 80$ & $0,11 \mathrm{E} 01$ \\
\hline westlake & & & 40 & $0,55 \mathrm{E} 04$ & $0,48 E 56$ & $0,12 \mathrm{E} 01$ \\
\hline
\end{tabular}

* delineamentos saturados

* valores expressos na forma exponencial de base 10 
TABELA 2 - Valores dos critérios A, D e E-ótịmos dos delineamentos compostos na região cuboidal e na situação 1 .

\begin{tabular}{|c|c|c|c|c|c|c|}
\hline \multirow[b]{2}{*}{ Del. } & \multirow[b]{2}{*}{ Dim. } & \multirow[b]{2}{*}{$\mathbf{P}$} & \multirow[b]{2}{*}{$\mathbf{N}$} & \multicolumn{3}{|c|}{ CRITÉRIOS ÓTIMOS** } \\
\hline & & & & $\mathbf{A}$ & D & $\mathbf{E}$ \\
\hline & 4 & 15 & & & & \\
\hline Hartley & & & 16 & $0,91 \mathrm{E} 02$ & $0,48 \mathrm{E} 08$ & $0,34 \mathrm{EOO}$ \\
\hline Draper-Iin & & & 16 & $0,91 \mathrm{E} 02$ & $0,48 \mathrm{E} 08$ & $0,34 \mathrm{EOO}$ \\
\hline Iucas* & & & 15 & $0,49 \mathrm{E} 03$ & $0,17 \mathrm{E} 16$ & $0,56 \mathrm{E} 00$ \\
\hline & 5 & 21 & & & & \\
\hline Hartley & & & 26 & $0,81 \mathrm{E02}$ & $0,30 \mathrm{E0}$ & $0,40 \mathrm{EO} 1$ \\
\hline Draper-Lin* & & & 21 & $0,26 \mathrm{E} 03$ & $0,92 \mathrm{E} 13$ & $0,36 \mathrm{E} 01$ \\
\hline Draper-Iin & & & 22 & $0,21 \mathrm{E} 03$ & $0,20 \mathrm{E} 13$ & $0,36 \mathrm{E} 01$ \\
\hline Iucas* & & & 21 & $0,11 \mathrm{E} 04$ & $0,57 \mathrm{E} 25$ & $0,17 \mathrm{E} 01$ \\
\hline westlake & & & 22 & $0,23 \mathrm{E03}$ & $0,77 \mathrm{E} 12$ & $0,38 \mathrm{E01}$ \\
\hline & 6 & 28 & & & & \\
\hline Hartley* & & & 28 & $0,17 \mathrm{E02}$ & $0,18 \mathrm{E} 17$ & $0,43 \mathrm{EO} 1$ \\
\hline Draper-Iin* & & & 28 & $0,17 \mathrm{E0} 2$ & $0,18 \mathrm{E} 17$ & $0,43 \mathrm{EO} 1$ \\
\hline Lucas* & & & 28 & $0,45 \mathrm{E} 01$ & $0,80 \mathrm{E} 37$ & $0,17 \mathrm{E} 01$ \\
\hline & 7 & 36 & & & & \\
\hline Hartley & & & 46 & $0,45 \mathrm{E} 03$ & $0,55 \mathrm{E} 20$ & $0,57 \mathrm{E01}$ \\
\hline Draper-Iin & & & 38 & $0,98 \mathrm{E} 03$ & $0,11 \mathrm{E} 24$ & $0,52 \mathrm{E01}$ \\
\hline Draper-Lin* & & & 36 & $0,28 \mathrm{E} 04$ & $0,30 \mathrm{E} 26$ & $0,52 \mathrm{E0} 1$ \\
\hline Lucas* & & & 36 & $0,36 \mathrm{E} 04$ & $0,65 \mathrm{E} 52$ & $0,16 \mathrm{E} 01$ \\
\hline Westlake & & & 40 & $0,54 \mathrm{E} 03$ & $0,80 \mathrm{E} 22$ & $0,57 \mathrm{E} 01$ \\
\hline
\end{tabular}

* delineamentos saturados

* valores expressos na forma exponencial de base 10 
experimentais, apresenta sob os aspectos da teoria ótima excelentes resultados, ou seja: para os parâmetros do modelo de segunda ordem estimados, este delineamento é o que possui - menor volume do elipsóide de confiança; todos os eixos deste elipsóide são estimados com uma precisão mais uniforme, ou seja, o elipsóide não apresenta eixos muito longos uns em relação aos outros e; permite estimativas de contrastes do tipo $a^{\prime} \beta$ sujeitos à restrição $a_{\sim}^{\prime} a=1$, com maior precisão. Na região cuboidal delineamento de Hartley também apresenta os melhores resultados dos critérios A, D e E-ótimos do que na região esférica, permitindo afirmar que estes critérios ótimos são afetados pela forma da região experimental, ou seja, das posições em que os níveis dos fatores são colocados. Nos casos em que ocorre equivalência entre os delineamentos de Hartley e Draper-Lin, a preferência fica para o delineamento de Draper-Lin por causa da facilidade de sua obtenção. Os maiores valores dos critérios ótimos A, D e E-ótimos, e portanto os piores resultados da teoria ótima, são apresentados pelo delineamento de Lucas, em todas as dimensões e nas duas regiões experimentais.

A Tabela 3 mostra os valores para os critérios A, D e E-ótimos dos delineamentos na região esférica e nas situações 2 e 3 .

Caso $k=4$. A equivalência entre os delineamentos de Hartley e Draper-Lin verificada na situação 1 não é tão intensa. o delineamento de Draper-Lin apresentavalores para os critérios A, D-ótimos levemente inferiores, enquanto que o valor do critério E-ótimo é bem menor. Os valores bem menores para os critérios A e D-ótimos do delineamento de Lucas devem ser atribuídos ao mal condicionamento da matriz $x^{\prime} X$ deste delineamento, neste caso.

caso $k=5$. Para esta dimensão o delineamento 
de Hartley apresenta os menores valores para os critérios A, D e E-ótimos, seguido pelos delineamentos de Draper-Lin 22 pontos e Draper-Lin saturado. Os valores para o critério Eótimo são bem semelhantes para estes delineamentos. Os baixos valores para os critérios A e D-ótimos dos delineamentos de Lucas e Westlake devem ser atribuídos ao mal condicionamento das matrizes $X^{\prime} X$ destes delineamentos para $k=5$.

Caso $k=6$. Os valores para os critérios $A$ e E-ótimos dos delineamentos de Hartley e Draper-Lin são iguais, o que indica a manutenção da equivalência entre estes delineamentos para estes critérios. Os valores para o critério D-ótimo são diferentes com o delineamento de DraperLin apresentando o menor valor. o baixo valor para o critério D-ótimo apresentado pelo delineamento de Lucas pode ser atribuido ao mal condicionamento da matriz $X^{\prime} X$ deste delineamento. Os valores para os critérios A e E-ótimos do delineamento de Lucas são superiores aos dos delineamentos de Hartley e Draper-Lin.

Caso $k=7$. Nesta dimensão o delineamento de Westlake apresenta os menores resultados para os três critérios ótimos enquanto que o delineamento de Hartley apresenta valores negativos para os critérios A e D-ótimos, o que pode ser atribuido a problemas de mal condionamento da matriz $X^{\prime} X$, juntamente com 0 delineamento de Lucas.

A Tabela 4 apresenta os valores para os critérios A, D e E-ótimos dos delineamentos compostos em estudo, nas situaçōes 2 e 3 para a região cuboidal.

Caso $k=4$. O delineamento de Draper-Lin apresenta os menores valores para os critérios A e D-ótimos, seguido pelo delineamento de Hartley. o baixo valor para o critério D-ótimo do delineamento de Lucas pode ser atribuido ao mal condicionamento de sua matriz $X^{\prime} X$. O delineamento de 
Valores dos critérios A, $D$ e E-ótimos dos delineamentos compostos, na região esférica e nas situações 2 e 3 .

\begin{tabular}{|c|c|c|c|c|c|c|}
\hline \multirow[b]{2}{*}{ Del. } & \multirow[b]{2}{*}{ Dim. } & \multirow[b]{2}{*}{$\mathbf{P}$} & \multirow[b]{2}{*}{$\mathbf{N}$} & \multicolumn{3}{|c|}{ CRITÉRIOS ÓTIMOS** } \\
\hline & & & & $\mathbf{A}$ & D & $\mathbf{E}$ \\
\hline & 4 & 15 & & & & \\
\hline Hartley & & & 16 & $0,21 \mathrm{E} 05$ & $0,13 E-07$ & $0,11 \mathrm{E} 04$ \\
\hline Draper-Lin & & & 16 & $0,19 \mathrm{E0} 5$ & $0,12 \mathrm{E}-07$ & $0,85 \mathrm{E} 03$ \\
\hline Lucas* & & & 15 & $-0,24 \mathrm{E} 06$ & $-0,26 E 34$ & $0,12 \mathrm{E} 06$ \\
\hline & 5 & 21 & & & & \\
\hline Hartley & & & 26 & $0,17 \mathrm{E} 05$ & $0,41 \mathrm{E} 40$ & $0,20 \mathrm{E} 02$ \\
\hline Draper-Lin* & & & 21 & $0,17 \mathrm{E} 06$ & $0,72 \mathrm{E} 44$ & $0,20 \mathrm{E} 02$ \\
\hline Draper-Lin & & & 22 & $0,73 \mathrm{E} 05$ & $0,13 \mathrm{E} 43$ & $0,19 \mathrm{E} 02$ \\
\hline Lucas* & & & 21 & $-0,28 \mathrm{E} 06$ & $-0,16 \mathrm{E} 54$ & $0,24 \mathrm{EO} 2$ \\
\hline westlake & & & 22 & $-0,10 \mathrm{E} 06$ & $-0,22 \mathrm{E} 46$ & $0,21 \mathrm{E} 02$ \\
\hline & 6 & 28 & & & & \\
\hline Hartley* & & & 28 & $0,29 \mathrm{E} 02$ & $0,14 \mathrm{E} 61$ & $0,26 \mathrm{EO} 2$ \\
\hline Draper-Lin* & & & 28 & $0,29 \mathrm{E} 02$ & $0,25 \mathrm{E} 61$ & $0,26 \mathrm{E} 02$ \\
\hline Lucas* & & & 28 & $0,33 \mathrm{E} 06$ & $-0,68 E 81$ & $0,31 \mathrm{E} 02$ \\
\hline & 7 & 36 & & & & \\
\hline Hartley & & & 46 & $-0,23 \mathrm{E} 06$ & $-0,15 \mathrm{E} 87$ & $0,34 \mathrm{E} 02$ \\
\hline Draper-Lin & & & 38 & $0,33 \mathrm{E06}$ & $0,20 E 87$ & $0,34 \mathrm{EO} 2$ \\
\hline Draper-Lin* & & & 36 & $0,39 \mathrm{E} 06$ & $0,35 \mathrm{E} 87$ & $0,34 \mathrm{EO} 2$ \\
\hline Lucas* & & & 36 & $0,67 \mathrm{E} 06$ & $-0,42 \mathrm{E} 112$ & $0,40 \mathrm{EO} 2$ \\
\hline Westlake & & & 40 & $0,21 \mathrm{E} 06$ & $0,56 \mathrm{E} 86$ & $0,34 \mathrm{E} 02$ \\
\hline
\end{tabular}

* delineamentos saturados

** valores expressos na forma exponencial de base 10 
TABELA 4 -

Valores dos critérios A, $D$ e E-ótimos delineamentos compostos na região cuboidal e nas situações 2 e 3 .

\begin{tabular}{|c|c|c|c|c|c|c|}
\hline \multirow[b]{2}{*}{ Del. } & \multirow[b]{2}{*}{ Dim. } & \multirow[b]{2}{*}{$\mathbf{P}$} & \multirow[b]{2}{*}{$\mathbf{N}$} & \multicolumn{3}{|c|}{ CRITÉRIOS ÓTIMOS** } \\
\hline & & & & $\mathbf{A}$ & D & $\mathbf{E}$ \\
\hline & 4 & 15 & & & & \\
\hline Hartley & & & 16 & $0,11 \mathrm{E} 05$ & $0,30 \mathrm{E} 17$ & $0,79 \mathrm{E}-01$ \\
\hline Draper-Iin & & & 16 & $0,11 \mathrm{E} 04$ & $0,83 \mathrm{E} 16$ & $0,82 \mathrm{E}-01$ \\
\hline \multirow[t]{2}{*}{ Lucas* } & & & 15 & $0,95 \mathrm{E} 05$ & $-0,52 E 29$ & $0,51 \mathrm{E}-01$ \\
\hline & 5 & 21 & & & & \\
\hline Hartley & & & 26 & $0,11 \mathrm{E} 04$ & $0,48 \mathrm{E} 19$ & $0,16 \mathrm{E} 02$ \\
\hline Draper-Lin* & & & 21 & $0,39 \mathrm{E} 05$ & $0,42 E 29$ & $0,16 \mathrm{E} 02$ \\
\hline Draper-Iin & & & 22 & $0,45 \mathrm{E} 04$ & $0,66 \mathrm{E} 24$ & $0,16 \mathrm{E} 02$ \\
\hline Lucas* & & & 21 & $0,29 \mathrm{E} 06$ & $0,32 \mathrm{E} 46$ & $0,27 \mathrm{E} 02$ \\
\hline \multirow[t]{2}{*}{ Westlake } & & & 22 & $0,13 \mathrm{E} 05$ & $0,98 \mathrm{E} 25$ & $0,18 \mathrm{E} 02$ \\
\hline & 6 & 28 & & & & \\
\hline Hartley* & & & 28 & $0,32 \mathrm{E} 02$ & $0,58 \mathrm{E} 35$ & $0,21 \mathrm{E} 02$ \\
\hline Draper-Lin* & & & 28 & $0,32 \mathrm{E} 02$ & $0,30 E 33$ & $0,20 \mathrm{E} 02$ \\
\hline \multirow[t]{2}{*}{ Iucas* } & & & 28 & $-0,38 \mathrm{E} 05$ & $-0,16 \mathrm{E} 66$ & $0,37 \mathrm{E} 02$ \\
\hline & 7 & 36 & & & & \\
\hline Hartley & & & 46 & $0,63 E 05$ & $0,61 \mathrm{E} 42$ & $0,25 \mathrm{E} 02$ \\
\hline Draper-Iin & & & 38 & $0,99 \mathrm{E} 04$ & $0,47 \mathrm{E} 43$ & $0,25 \mathrm{E} 02$ \\
\hline Draper-Lin* & & & 36 & $0,67 \mathrm{E} 05$ & $0,14 \mathrm{E} 47$ & $0,25 \mathrm{E} 02$ \\
\hline Iucas* & & & 36 & $0,35 \mathrm{E06}$ & $0,41 E 90$ & $0,45 \mathrm{E} 02$ \\
\hline Westlake & & & 40 & $0,67 \mathrm{E} 04$ & $0,46 \mathrm{E} 41$ & $0,24 \mathrm{E02}$ \\
\hline
\end{tabular}

* delineamentos saturados

* valores expressos na forma exponencial de base 10 
Lucas apresenta o menor valor para o critério E-ótimo, seguidos pelos valores dos delineamentos de Hartley e DraperLin.

Caso $k=5$. Observa-se dos resultados que 0 delineamento de Hartley apresenta os menores valores para os três critérios. Logo a seguir, tem-se os delineamentos de Draper-Lin 22 pontos, Westlake e Draper-Lin saturado, quanto aos critérios A e D-ótimos. Os valores do critério E-ótimo dos delineamentos de Hartley, Draper-Lin saturado e DraperLin 38 pontos apresentam uma equivalência. O delineamento de Lucas apresenta os maiores resultados para os critérios A, D e E-ótimos.

Caso $k=6$. Neste caso, os delineamentos de Hartley e Draper-Lin apresentam os mesmos valores para os critérios A e E-ótimos. Os resultados para o critério D-ótimo foram diferentes com o delineamento de Draper-Lin apresentando o menor valor. Novamente, os baixos valores dos critérios A e D-ótimos do delineamento de Lucas podem ser atribuídos ao mal condicionamento de sua matriz $x^{\prime} x$, neste caso.

Caso $k=7$. Tal como se verificou na região esférica o delineamento de Westlake apresentou os menores resultados para os três critérios ótimos. Os valores para o critério E-ótimo mostram uma equivalência entre os delineamentos de Hartley e Draper-Lin. Os maiores valores para os critérios A, D e E-ótimos são apresentadas pelo delineamento de Lucas.

Assim, os delineamentos de Hartley e DraperLin apresentam os melhores resultados dos critérios A, D e Eótimos para $k=4,5$ e 6 fatores nas duas regiōes experimentais. Para $\mathrm{k}=7$ surge o delineamento de Westlake com os melhores resultados dos critérios A, D e E-ótimos, nas 
duas regiōes experimentais. Este delineamento tem 4 pontos experimentais a mais do que o número de parâmetros a serem estimados do modelo polinomial de 2 a ordem. os pior desempenho, principalmente quanto ao critério D-ótimo, é apresentado pelo delineamento de Lucas, nas duas regiōes experimentais e para todas as dimensōes. 0 mal condicionamento da matriz $X^{\prime} X$ deste delineamento é devido ao valor de $\alpha$.

\subsection{Porcentagem de reprodução do ponto crítico (\%Rep) e número médio de inversões (MMinv).}

As Tabelas 5, 6, 7 e 8 apresentam os valores da \%Rep e do NMinv dos delineamentos compostos pequenos, para $\mathrm{k}=4$, 5, 6, 7 fatores, respectivamente, nas regiōes experimentais esférica e cuboidal, na situação 1 , com valores de $\sigma=100,250$ e 300, correspondendo aproximadamente a coeficientes de variação de $2,5 \%, 7 \%$ e $8,5 \%$, respectivamente.

Caso $k=4$. Os resultados da Tabela 5 mostram que os delineamentos de Hartley e Draper-Lin apresentam praticamente os mesmos valores da \%Rep do ponto de máximo e do NMinv nas duas regiōes experimentais, e para os três valores de $\sigma$. As pequenas diferenças verificadas são devidas a aleatorização do processo de simulação de dados. Estes resultados confirmam a equivalência destes delineamentos verificada na seção 4.1 , para 4 fatores. Os menores valores de \%Rep do ponto de máximo e os maiores valores do NMinv são apresentados pelos delineamentos de Lucas para $\sigma=250$ e 300 .

Caso $k=5$. Os resultados apresentados na Tabela 6 mostram que o delineamento de Hartley tem os maiores valores da \%Rep do ponto de máximo e os menores valores de 
NMinv nas duas regiōes, para os três valores de $\sigma$. Na região cuboidal, somente a \%Rep do ponto de máximo do delineamento de Lucas decresce com o aumento do coeficiente de variação, todos os outros delineamentos apresentam altos valores da \%Rep do ponto de máximo para os três valores de $\sigma$.

Caso $k=6$. Os resultados da Tabela 7 mostram novamente, a equivalência entre os delineamentos de Hartley e Draper-Lin, nos três valores de c.v. e nas duas regiōes. o delineamento de Lucas apresenta os menores valores da \%Rep do ponto de máximo e os maiores valores do NMinv.

Caso $k=7$. De acordo com os resultados da Tabela 8, na região esférica, o delineamento de Hartley apresenta o maior resultado da \%Rep do ponto de máximo e o menor valor do NMinv. Percebe-se que os valores da \%Rep do ponto de máximo de todos os delineamentos decrescem com o aumento do valor da variabilidade, enquanto que o valor do NMinv aumenta. Ainda nesta região, o delineamento de Lucas apresenta os menores valores da \%Rep do ponto de máximo e os maiores valores do NMinv para os três valores de $\sigma$. Na região cuboidal os delineamentos de Hartley e Westlake apresentam os maiores resultados da \%Rep do ponto de máximo e do NMinv para os três valores de $\sigma$, com ligeira predominância para delineamento de Hartley. Os delineamentos saturados de Draper-Lin e Lucas apresentam baixos valores da \%Rep do ponto de máximo $e$, cosequentemente altos valores de NMinv para valores de $\sigma=250$ e 300 .

Nesta situação em que as formas dos modelos reais, geradores das observaçōes simuladas, são iguais às formas dos modelos estimados, os delineamentos de Hartley e Draper-Lin apresentam as melhores porcentagens de reprodução do ponto de máximo, nas duas regiōes experimentais e para todas as dimensōes. 


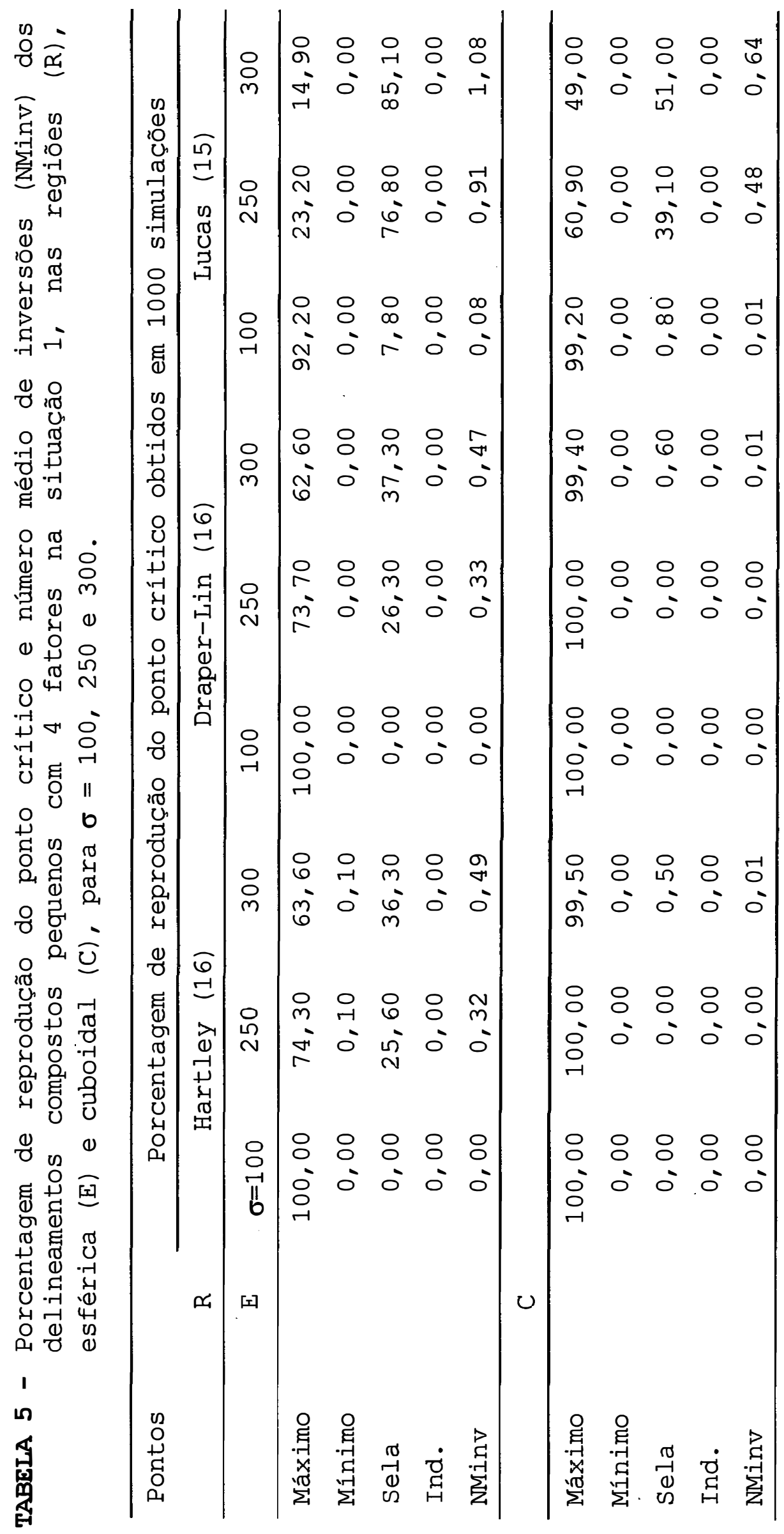




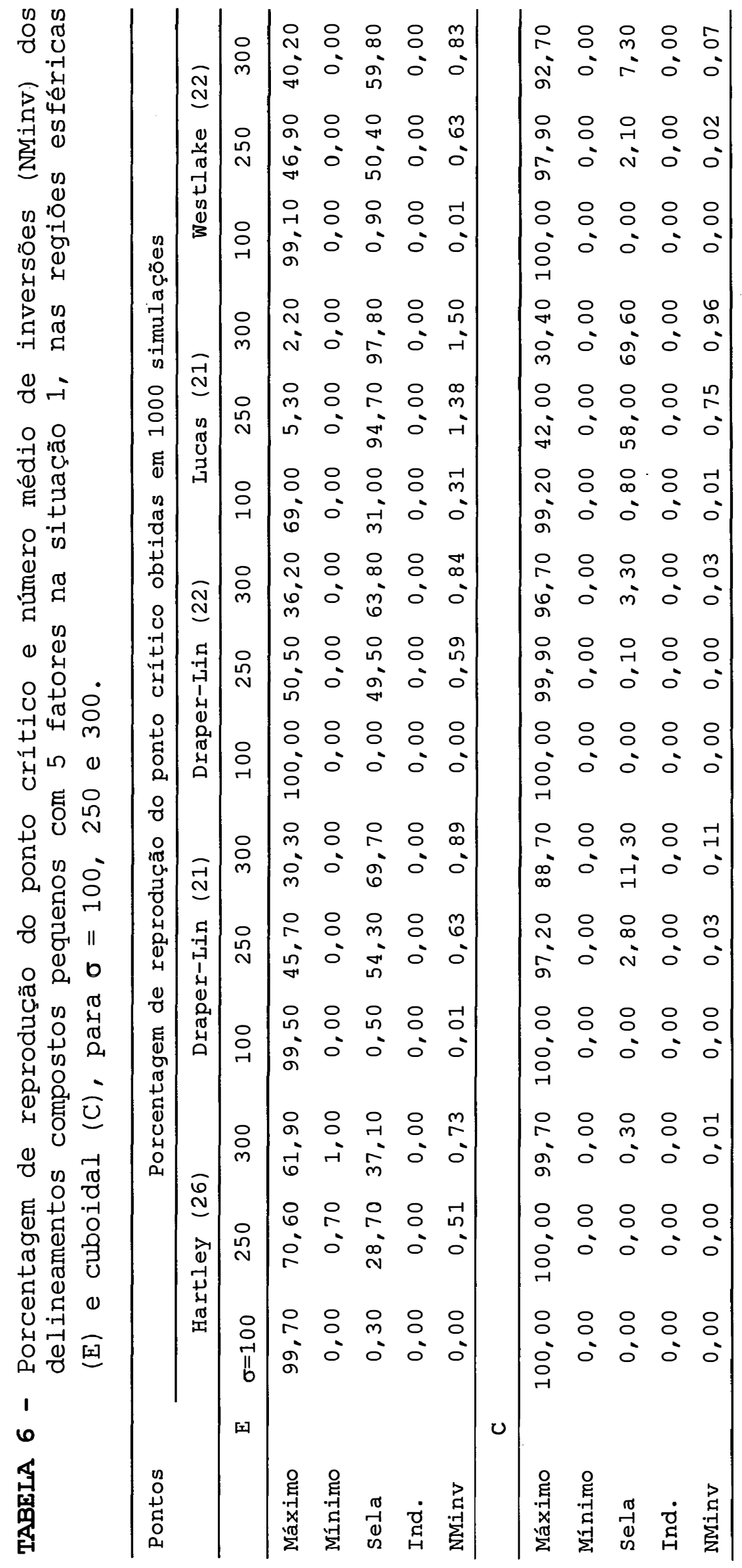




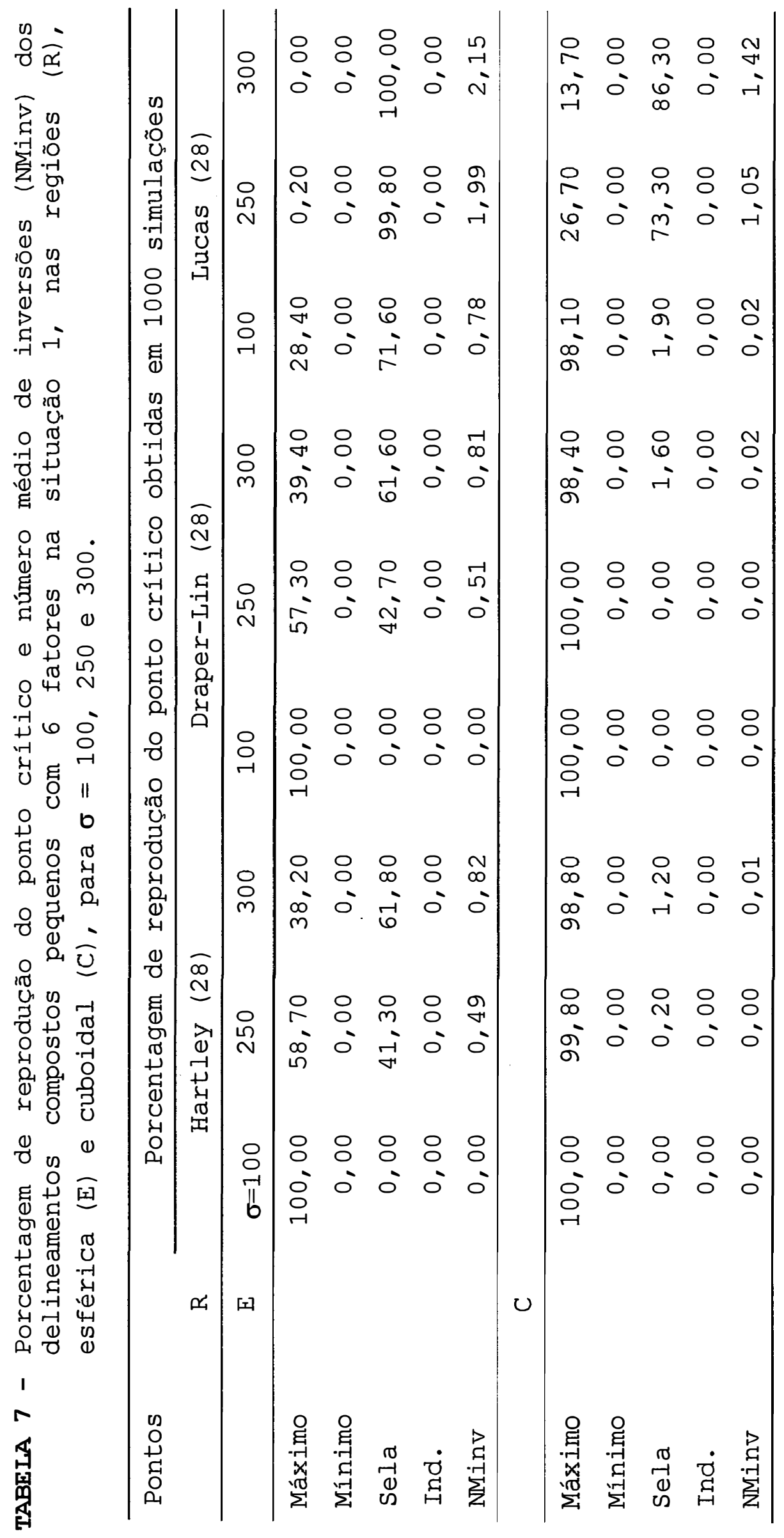




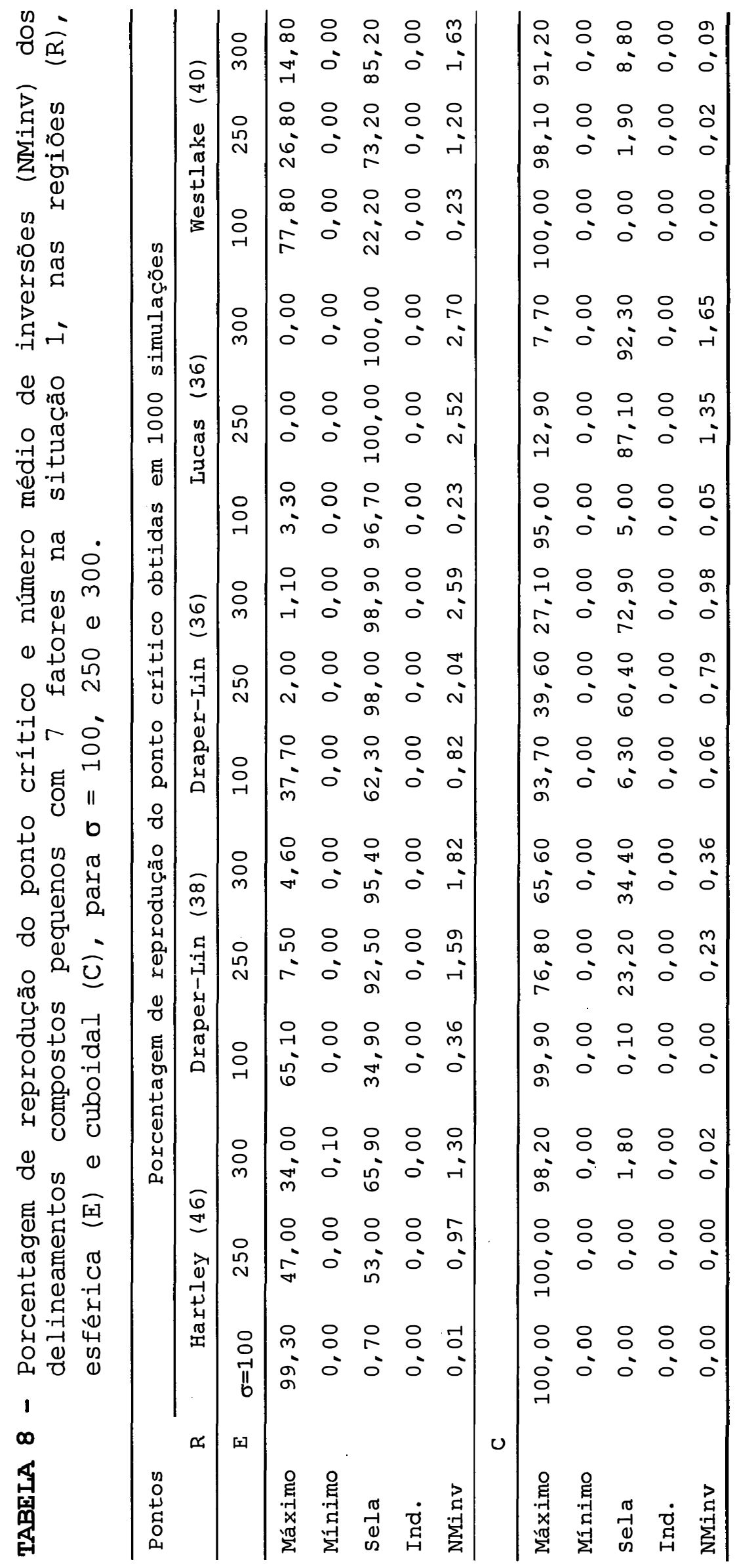


Para todos os delineamentos os valores da \%Rep do ponto de máximo decresce com o aumento do valor do coeficiente de variação, principalmente na região experimental esférica. Na região experimental cuboidal este decréscimo é mais suave. Nas dimensões de $k=5$ e 7 , além do delineamento de Hartley, destaca-se 0 delineamento de Westlake com excelentes resultados da \%Rep do ponto de máximo. Nenhum delineamento composto pequeno apresenta, inversão total de natureza do ponto ótimo, isto é, nenhum delineamento apresenta valores da \%Rep do ponto de mínimo.

o conjunto de Tabelas 9, 10, 11 e 12 apresenta os valores da \%Rep do ponto crítico e NMinv dos delineamentos em estudo, nas regióes experimentais esférica e cuboidal, na situação 2, com $\sigma=100,250$ e 300, para 4, 5, 6 e 7 fatores.

Caso $k=4$. Os resultados da Tabela 9 mostram que, na região esférica, os valores da \%Rep do ponto de máximo, são muito baixos. Os delineamentos de Hartley e Draper-Lin apresentam valores da \%Rep do ponto de minimo bastante significativos para os três valores de $\sigma$, indicando que a utilização destes delineamentos compostos pequenos, nesta situação, podem levar a uma inversão total da natureza do ponto crítico com uma razoável probabilidade. Os valores de \%Rep do ponto de sela são bem elevados para todos os delineamentos nos três valores de $\sigma$. Observa-se que o delineamento de Lucas apresenta os menores valores de NMinv. Na região cuboidal os resultados da \%Rep do ponto de máximo são um pouco melhores, principalmente para $\sigma=100$. Não se observa valores da \%Rep do ponto de mínimo. o valor do NMinv na região cuboidal é bem inferior ao valor da região esférica para todos os delineamentos e para todos os valores de $\sigma$. Observa-se, também, a equivalência entre os delineamentos de Hartley e Draper-Lin nas duas regiões e para os três valores 
de $\sigma$.

Caso $k=5$. Para a região esférica, a Tabela 10 mostra que os valores da \%Rep do ponto de máximo são muito baixos para quase todos os delineamentos. A exceção fica para os valores da \%Rep do ponto de máximo apresentados pelo delineamento de Westlake nos três valores de $\sigma$. Chama a atenção os altos valores da \%Rep do ponto de mínimo apresentados pelo delineamento de Hartley para os três valores de $\sigma$. Os delineamentos de Draper-Lin saturado, Draper-Lin 22 pontos e Lucas apresentam altos valores da \%Rep do ponto de sela. o delineamento de Westlake apresenta os menores resultados do NMinv. Na região cuboidal, quase todos os delineamentos apresentam altos valores da \%Rep do ponto de máximo quando $\sigma=100$, e diminuem na medida que o valor de $\sigma$ aumenta, a exceção do delineamento de Lucas, que apresenta baixos valores da \%Rep do ponto de máximo.

Caso $k=6$. Para a região esférica os resultados da Tabela 11 mostram baixos valores da \%Rep do ponto de máximo e altos valores da \%Rep de ponto de sela, o que reflete um fraco desempenho dos delineamentos compostos pequenos em estudo para reproduzir o ponto de máximo idealizado na seção 3.8.2. O delineamento de Lucas apresenta os menores valores do NMinv. Na região cuboidal quase todos os delineamentos apresentam altos valores de \%Rep do ponto de máximo para $\sigma=100$. Mas, à medida que $\sigma$ aumenta o valor da \%Rep do ponto de máximo diminui rapidamente e a \%Rep do ponto de sela aumenta consideravelmente. o delineamento de Lucas apresenta os menores valores da \%Rep do ponto de máximo e os maiores valores do NMinv para os três valores de $\sigma$. Confirmase a equivalência entre os delineamentos de Hartley e DraperLin quanto aos valores da \%Rep do ponto de máximo e NMinv. Caso $k=7$. Novamente repete-se a tendência 


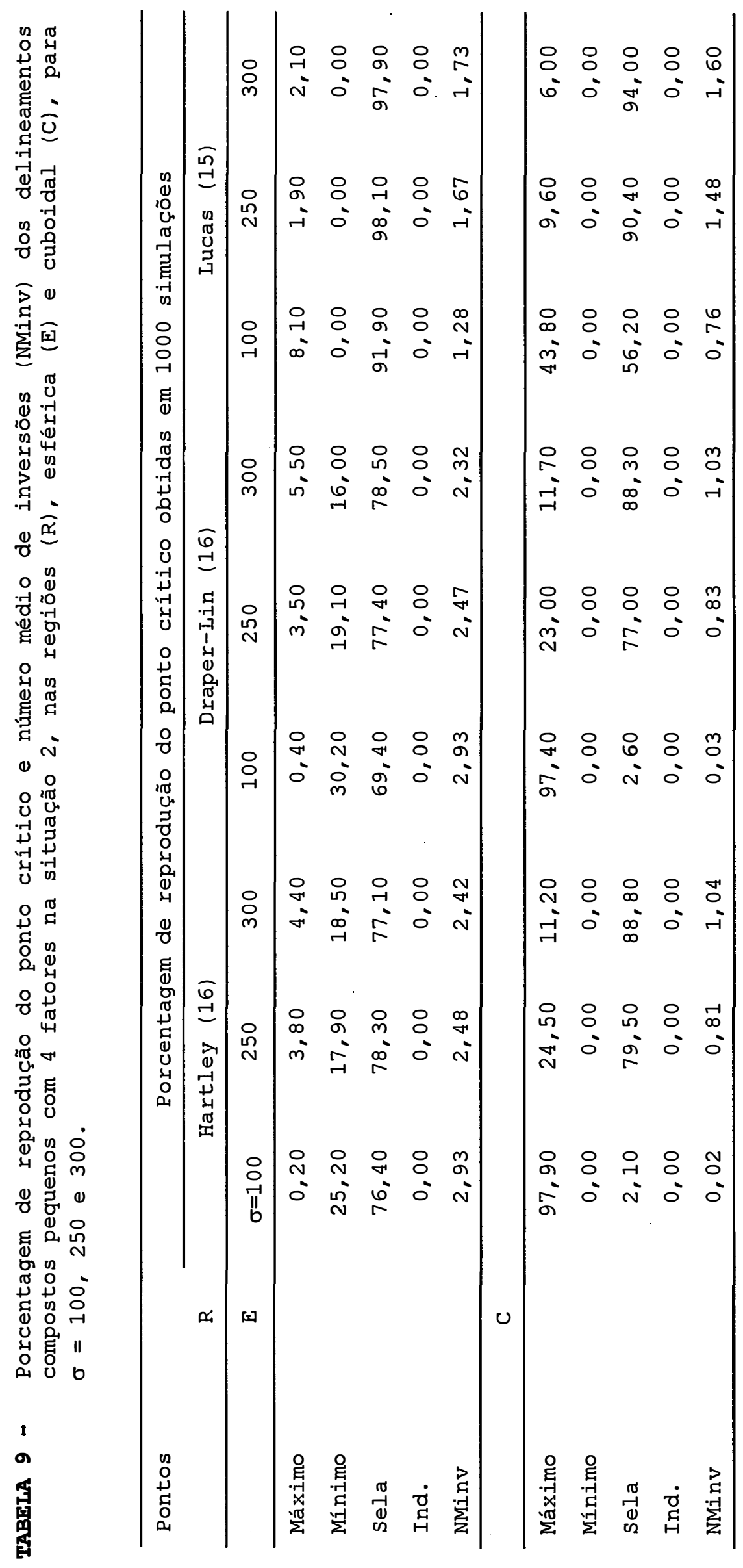




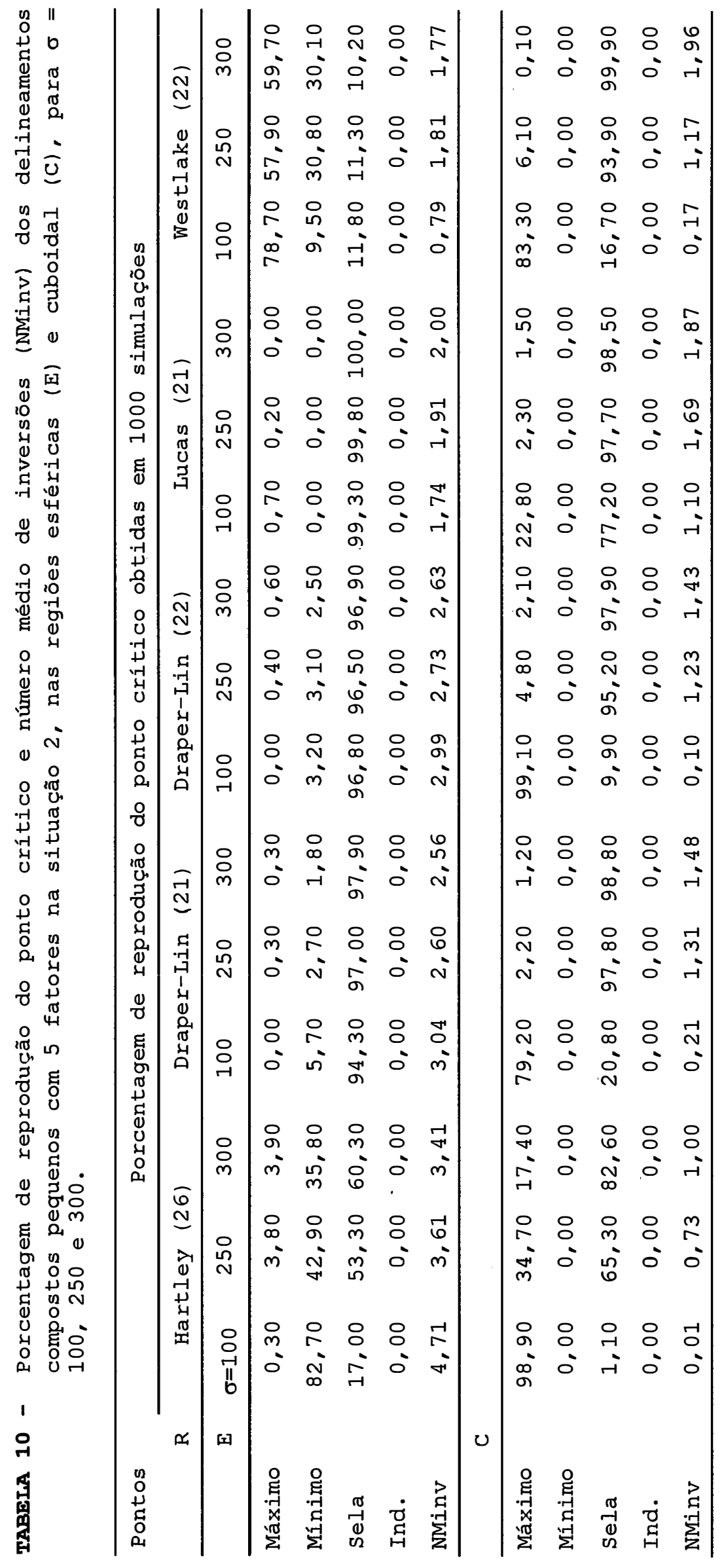




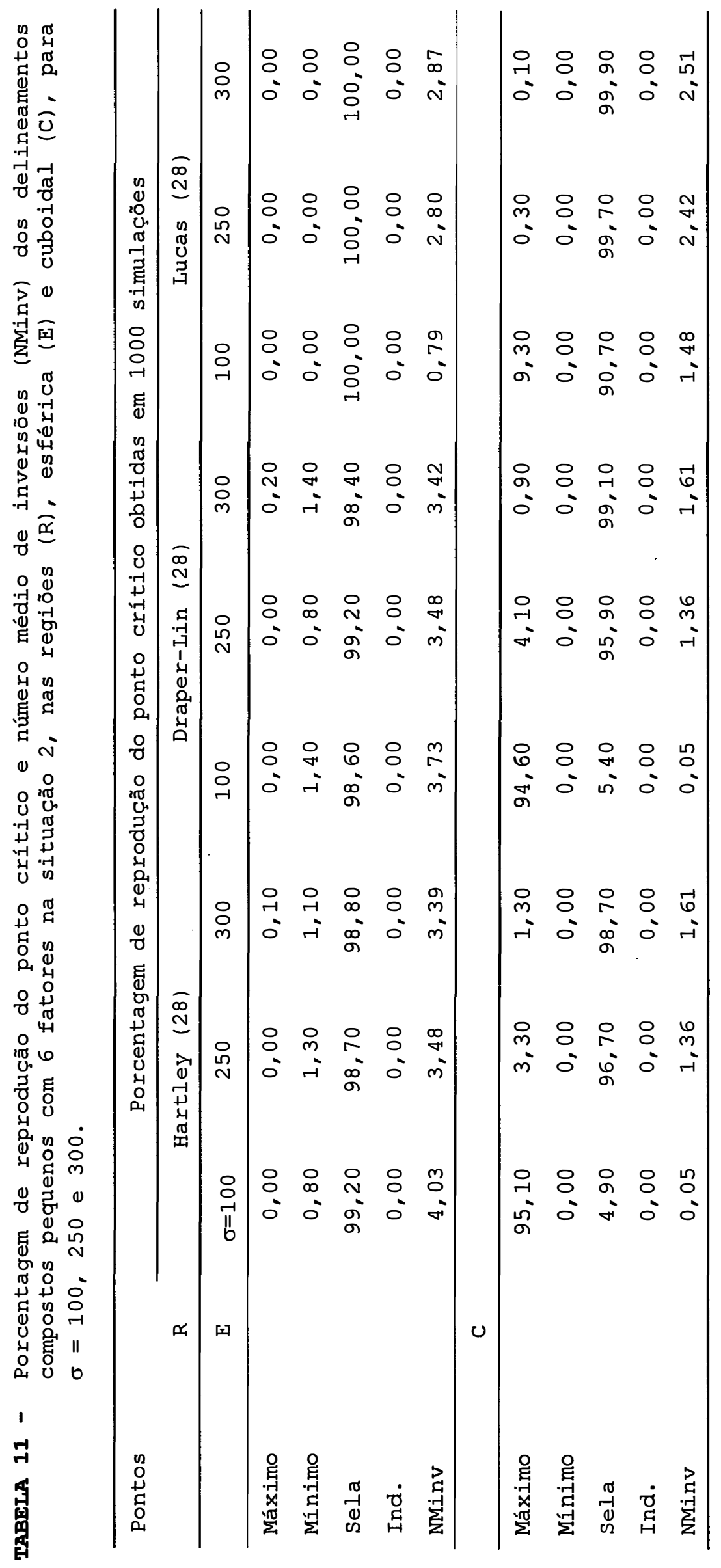




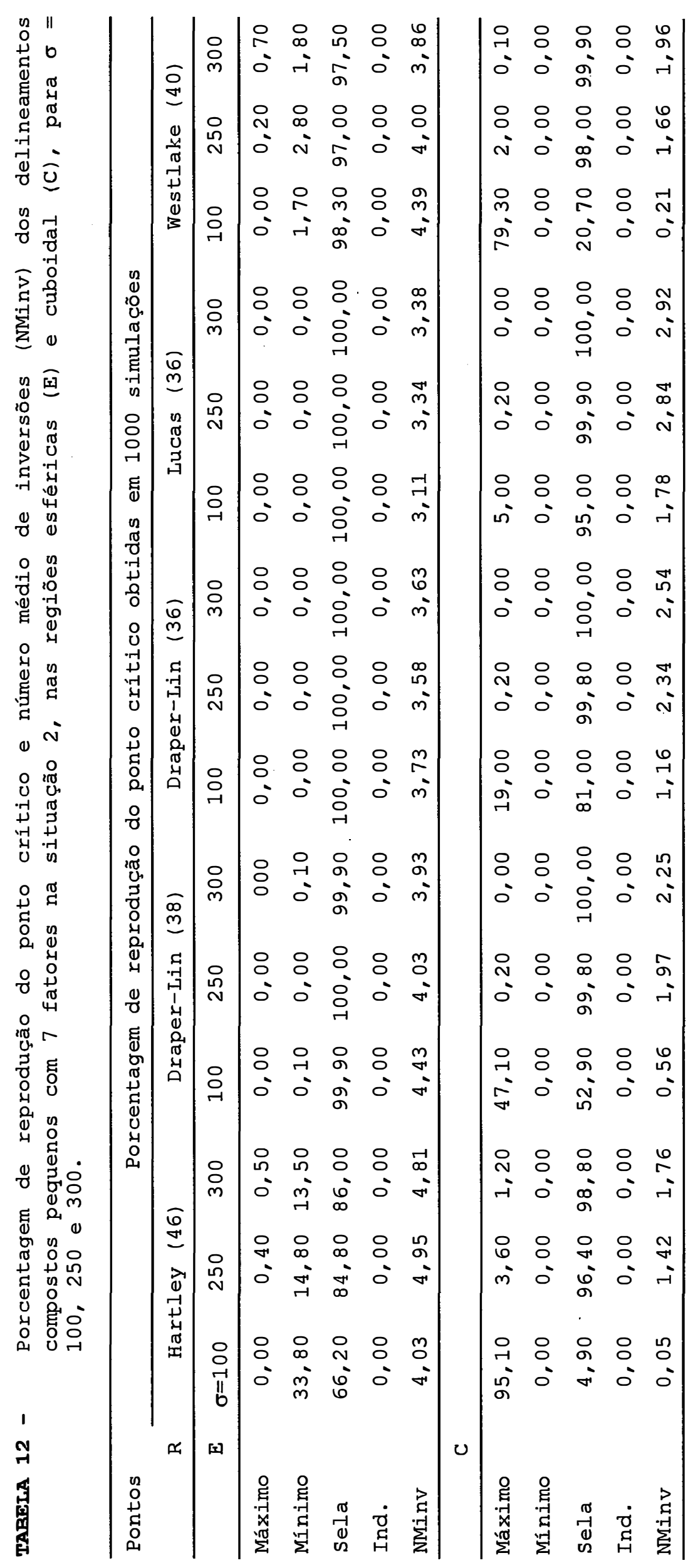


verificada no caso de $k=5$ desta situação na região esférica. A Tabela 12 apresenta valores da \%Rep do ponto de máximo muito baixos para todos os delineamentos nos três valores de $\sigma$. O delineamento de Hartley apresenta altos valores da \%Rep do ponto de mínimo, o que configura uma forte tendência de inversão da natureza do ponto critico idealizado na seção 3.9.1. Os outros delineamentos apresentam altos valores da \%Rep do ponto de sela. Chama a atenção os altos valores do NMinv dos delineamentos nesta região. Na região cuboidal os valores da \%Rep do ponto máximo dos delineamentos de Hartley e Draper-Lin são maior para $\sigma=100$, e na medida que $\sigma$ aumenta, os valores da \%Rep do ponto de máximo diminuem rapidamente, enquanto que o valor da \%Rep do ponto de sela aumenta. Os valores do NMinv são inferiores aos da região esférica.

Nesta situação em que há diferenças nas formas dos modelos reais e estimados, na região experimental esférica, em todas as dimensões e para todos os valores $\sigma$, os delineamentos compostos pequenos estudados apresentam um fraco desempenho na reprodução do ponto critico, juntamente com valores de NMinv muito elevados. O delineamento de Hartley apresenta altos valores da \%Rep do ponto de mínimo nas dimensões $k=4,5$ e 7 e todos os valores de c.v., significando que este delineamento, nestas condições pode inverter totalmente a natureza do ponto critico. Na região cuboidal o desempenho dos delineamentos melhora para c.v. baixos e piora rapidamente com $o$ aumento da variabilidade e com $\circ$ aumento da dimensão. O destaque para $k=5$ e 7 , nas duas reglóes experimentais, é o delineamento de Westlake que apresenta um bom desempenho na reprodução do ponto de máximo.

As Tabelas 13, 14, 15 e 16 apresentam os resultados da \%Rep do ponto crítico e o NMinv dos delineamentos compostos nas regiões esférica e cuboidal, na 


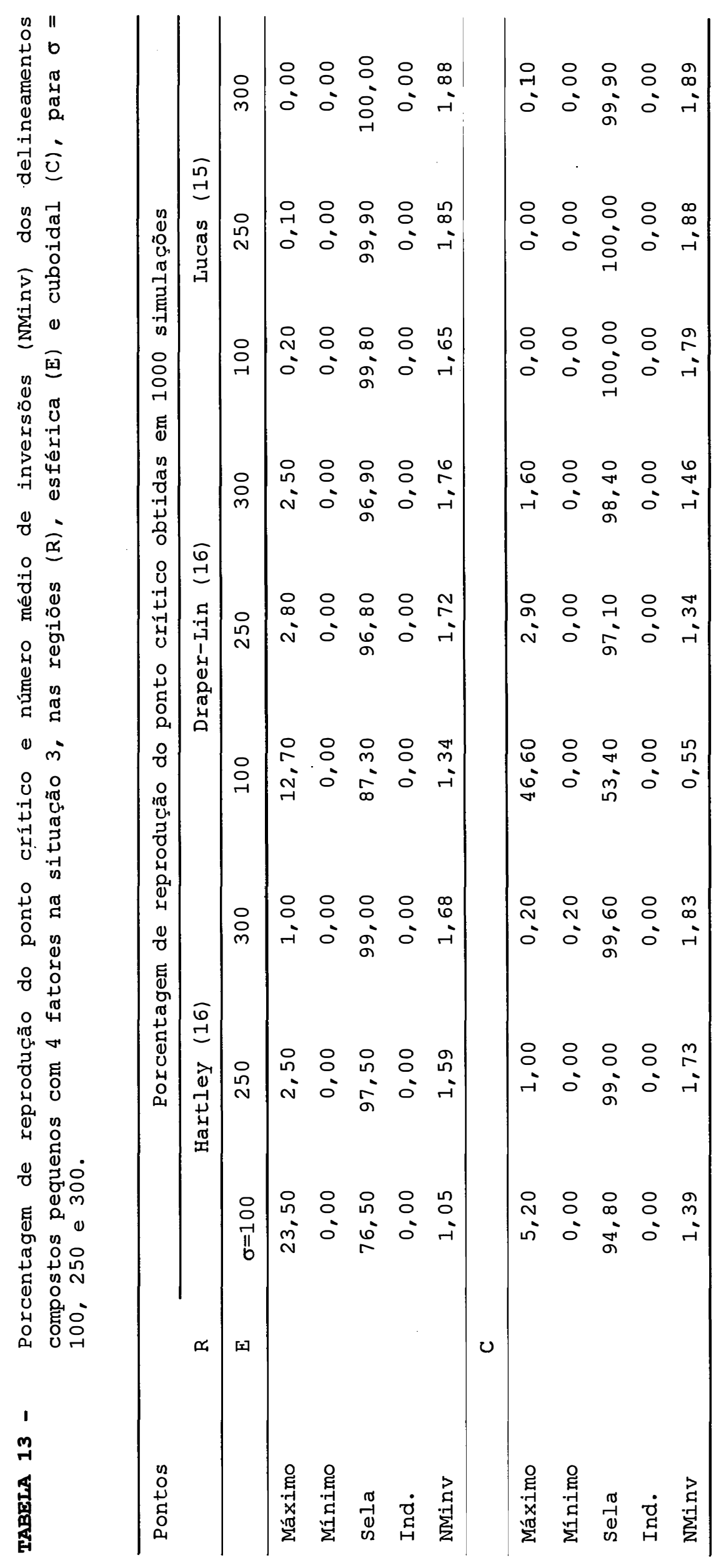




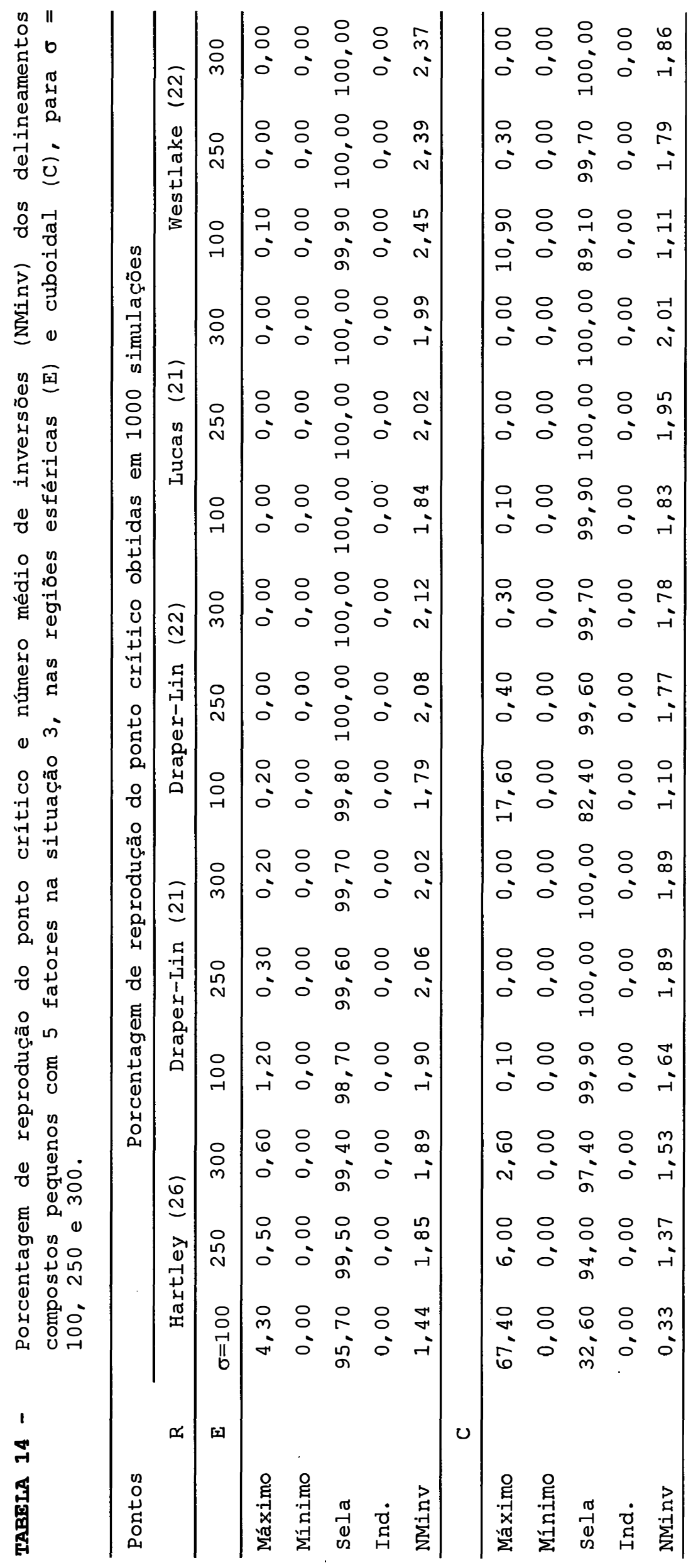




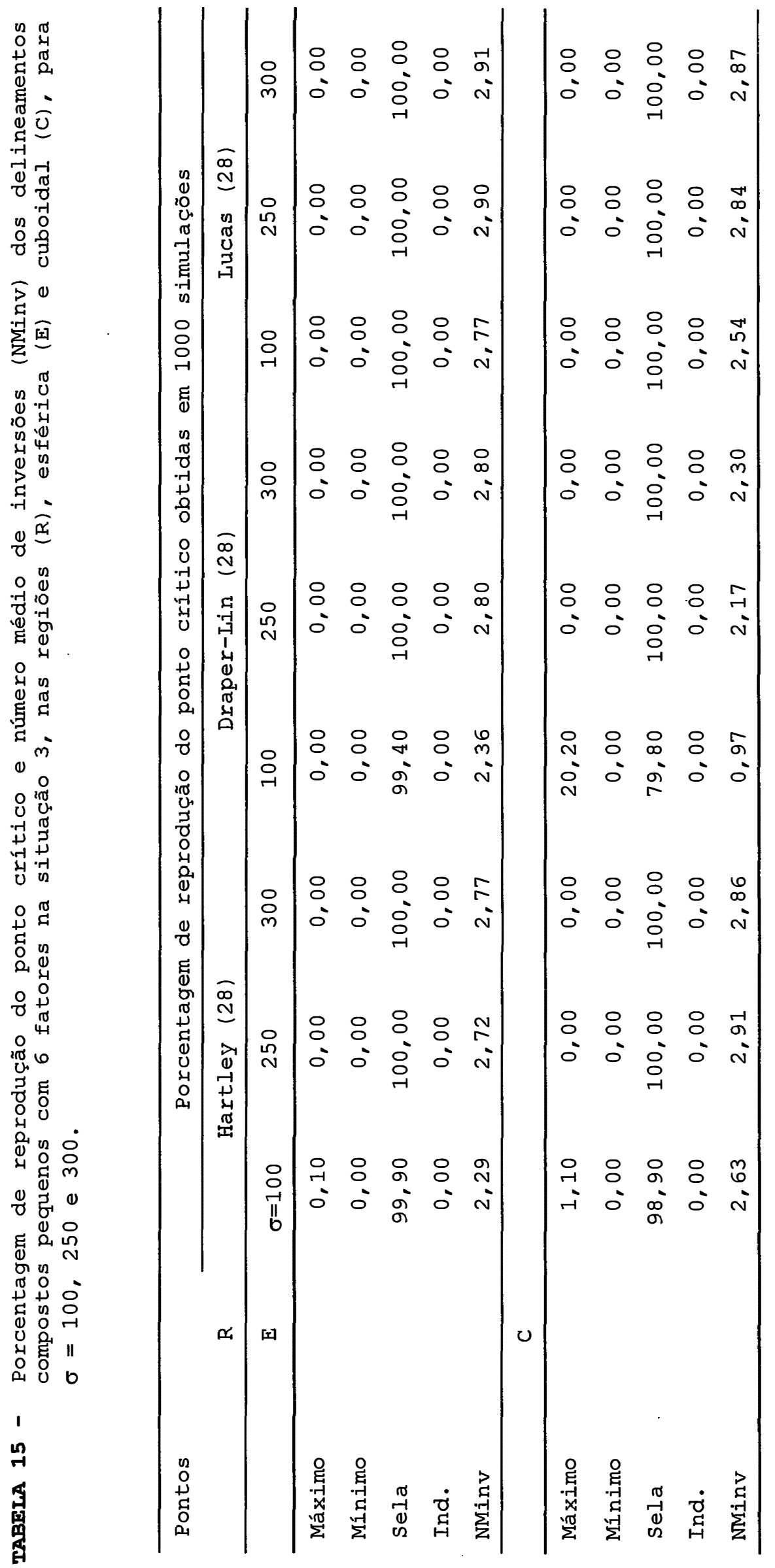




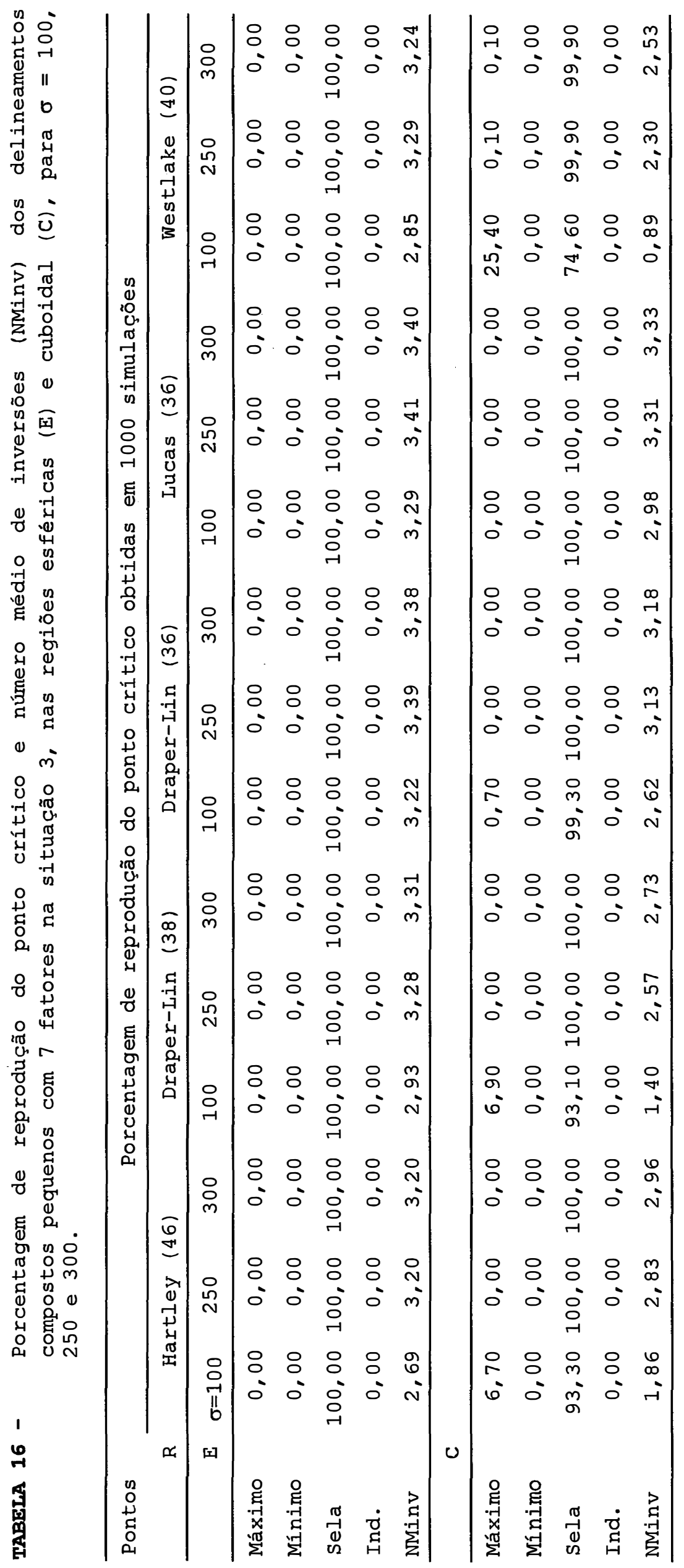


situação 3, para os três valores de $\sigma=100,250$ e 300 e para $4,5,6$ e 7 fatores.

Caso $k=4$. Os dados da Tabela 13 mostram que os delineamentos apresentam baixos valores da \%Rep do ponto de máximo e altos valores da \%Rep do ponto de sela, a medida que $o$ valor de $\sigma$ aumenta e nas duas regióes. Na região cuboidal e para $\sigma=100$, o delineamento de Draper-Lin apresenta um valor da \%Rep do ponto de máximo mais elevado.

Caso $k=5$. Os resultados apresentados pela Tabela 14 mostram baixos valores da \%Rep do ponto de máximo e altos valores da \%Rep do ponto de sela dos delineamentos, quando se aumenta $o$ valor de $\sigma$, nas duas regiões. 0 delineamento de Hartley apresenta um valor da \%Rep do ponto de máximo bem superior aos demais delineamentos na regiāo cuboidal com $\sigma=100$.

Caso $k=6$. Todos os resultados apresentados na Tabela 15 mostram altos valores da \%Rep do ponto de sela para todos os valores de $\sigma$ e nas duas regióes. Os valores do NMinv são bastantes homogêneos, dificultando a escolha de um delineamento na situação 3 com 6 fatores.

Caso $k=7$. Pelos resultados da Tabela 16, todos os delineamentos apresentam altos valores da \%Rep do ponto de sela, nas duas regiões e para todas os três valores de $\sigma$. O delineamento de Westlake apresenta o menor valor de NMinv para $\sigma=100$.

Para esta situação em que não há diferença entre as formas dos modelos reais e estimados, todos os delineamentos compostos pequenos apresentam alto valor da \%Rep do ponto de sela e um fraco desempenho em reproduzir o ponto de máximo nas duas regiões experimentais, para todas as dimensões $e$, para todos os valores de $\sigma$. Isto é um indicativo de que os delineamentos compostos pequenos estudados neste 
trabalho não se adaptam muito bem com o modelo polinomial em raiz quadrada em $x_{i}$. Fica muito difícil escolher um delineamento através do NMinv para esta situação, pois todos os delineamentos apresentam praticamente os mesmos valores de NMinv.

As Tabelas 17, 18, 19 e 20 mostram os resultados da \%Rep do ponto ótimo e NMinv dos delineamentos estudados, na situação 4 , para os três valores de $\sigma$ e para 4, 5, 6 e 7 fatores.

Caso $k=4$. Para a região esférica, os resultados da Tabela 17 mostram que os delineamentos de Hartley e Draper-Lin apresentam baixos valores da \%Rep do ponto de máximo e altos valores da \%Rep do ponto de sela. 0 valor mais alto da \%Rep do ponto de máximo é apresentado pelo delineamento de Lucas quando $\sigma=100$. Todos os delineamentos apresentam uma tendência de diminuição da \%Rep do ponto de máximo com o aumento de $\sigma$. Na região cuboidal, o delineamento de Hartley e Lucas apresentam os maiores valores da \%Rep do ponto de máximo, com uma pequena predominância do delineamento de Hartley. Os valores mais baixos foram apresentados pelo delineamento de Draper-Lin. Os valores do NMinv apresentados pelos delineamentos na região cuboidal são inferiores aos da região esférica e, estes aumentam na medida que $\sigma$ aumenta.

Caso $k=5$. Os resultados da Tabela 18 mostram que quase todos os delineamentos apresentam baixos valores da \%Rep do ponto de máximo, com os valores do delineamento de Westlake sendo levemente superiores, para a região esférica. $\mathrm{Na}$ região cuboidal, os delineamentos de Hartley, Lucas e Westlake apresentam os valores mais elevados para a \%Rep do ponto de máximo para pequenos valores de $\sigma$ e diminuem quando o valor de $\sigma$ aumenta. o delineamento de Hartley apresenta o 


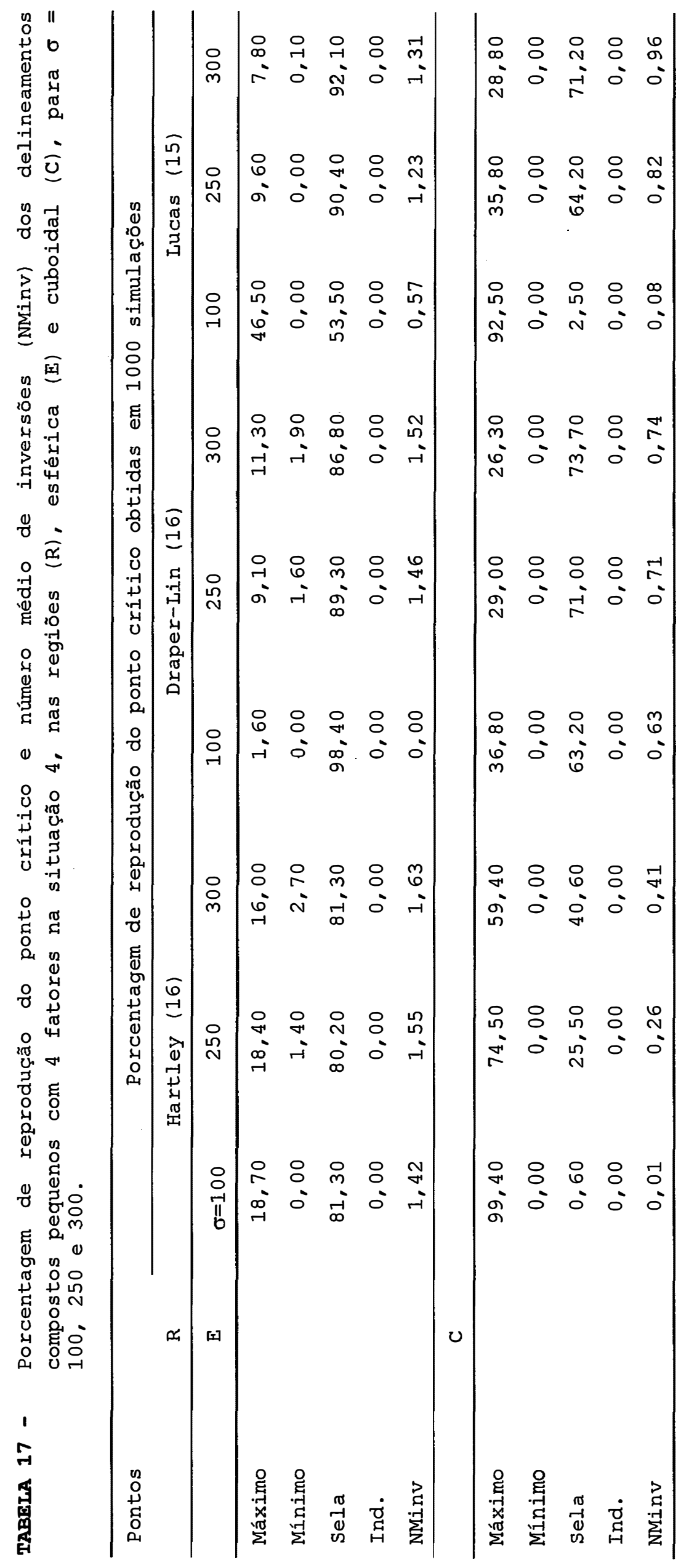




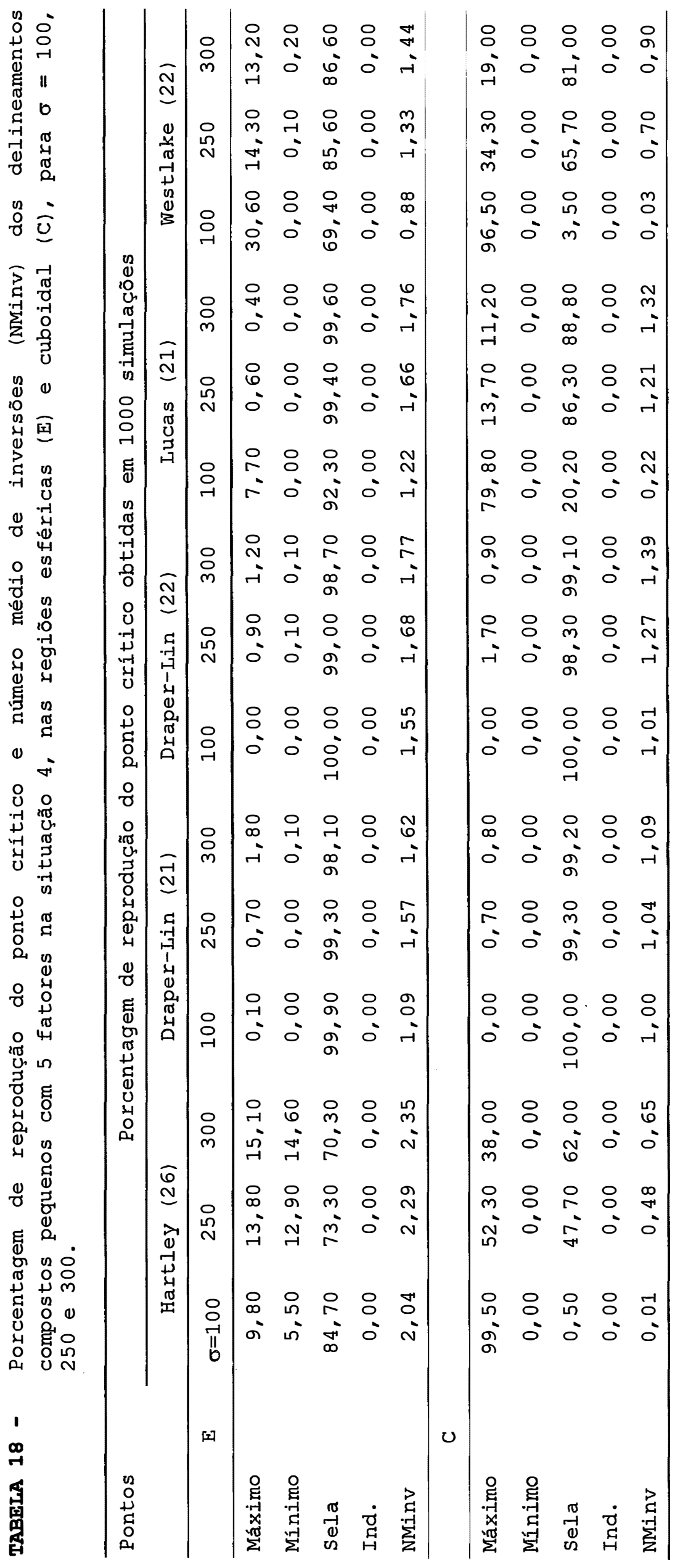




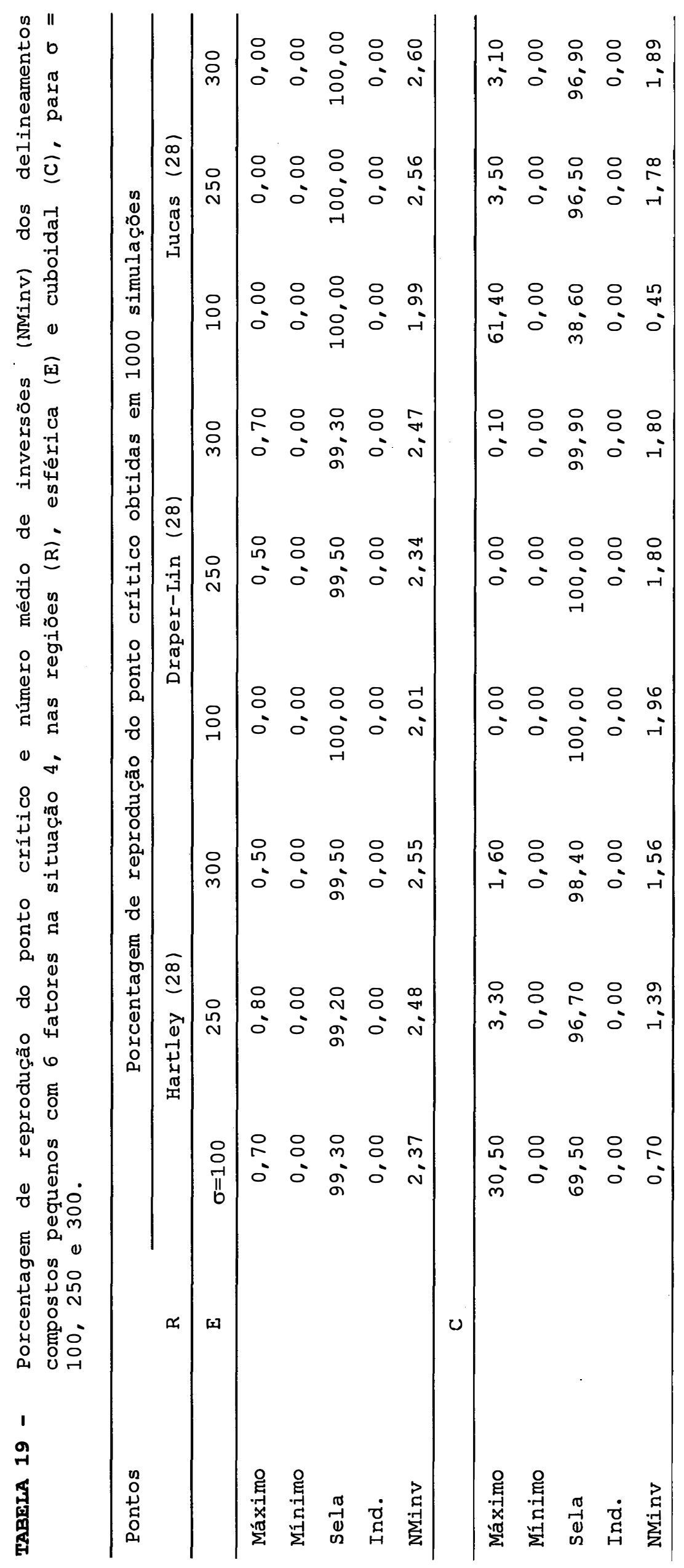




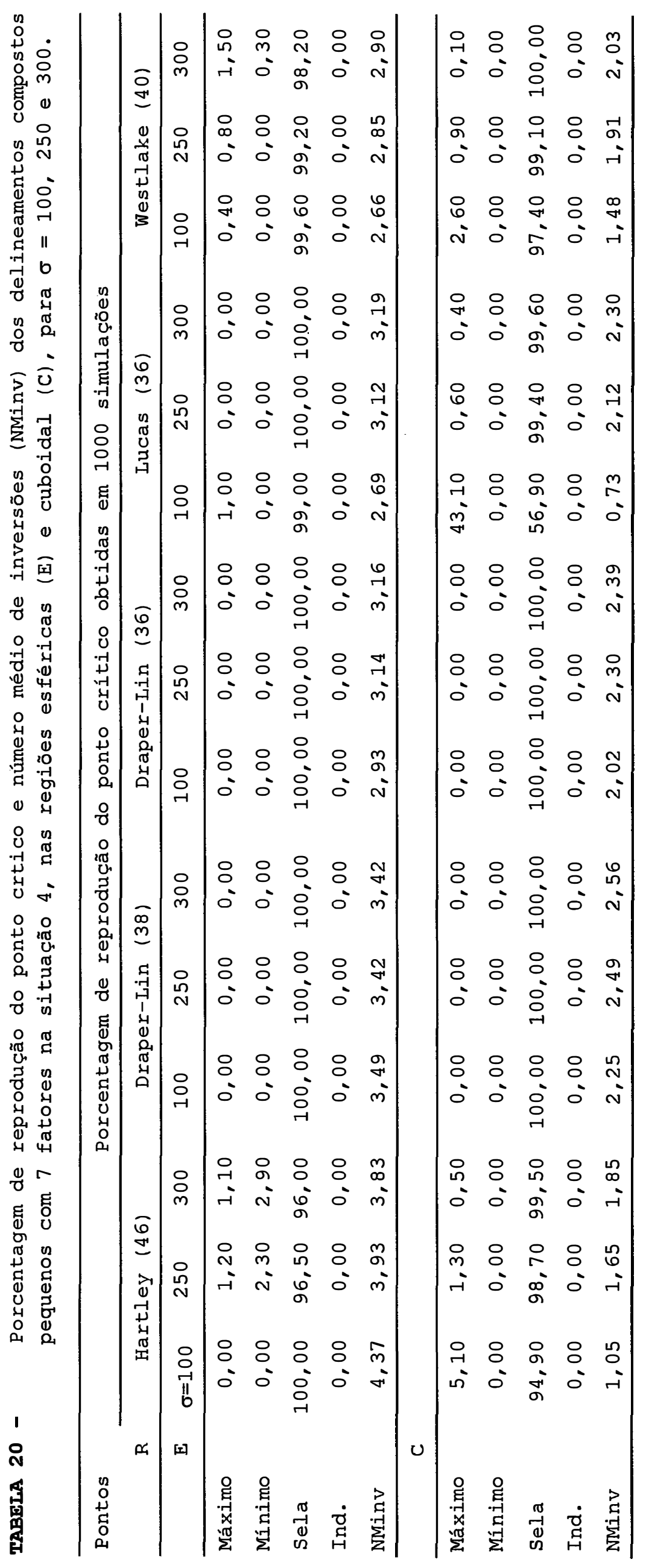


maior valor do NMinv na região esférica e o menor valor de NMinv na região cuboidal.

Caso $k=6$. Os resultados da Tabela 19 mostram que os três delineamentos apresentam, na região esférica, baixos valores da \%Rep do ponto de máximo. O delineamento de Lucas apresenta o menor valor do NMinv para $\sigma=100$. Na região cuboidal, o maior valor da \%Rep do ponto de máximo é apresentado pelo delineamento de Lucas para $\sigma=100$, enquanto que os outros delineamentos apresentam baixos valores. Nas duas regiōes, o NMinv aumenta quando $\sigma$ aumenta.

Caso $k=7$. Os resultados da Tabela 20 mostram que os delineamentos compostos pequenos apresentam, na região esférica, altos valores da \%Rep do ponto de sela e um alto valor do NMinv. Na região cuboidal, o delineamento de Lucas apresenta um valor da \%Rep do ponto de máximo mais elevado que os demais delineamentos, para $\sigma=100$. Os outros delineamentos apresentam altos valores da \%Rep do ponto de sela. Na região esférica o valor do NMinv é superior ao valor do NMinv apresentado na região cuboidal.

Nesta situação em que as formas dos modelos reais e estimados são diferentes, o desempenho dos delineamentos difere para as duas regiōes experimentais, com - melhor desempenho destes na região cuboidal, sendo que o valor da \%Rep do ponto de máximo dos delineamentos decresce com o aumento do coeficiente de variação. Para c.v. em torno de $2,5 \%$ e na região cuboidal, os delineamentos compostos com - melhor desempenho para esta situação foram: os de Hartley e Lucas para $k=4$ e, os de Hartley, Lucas e Westlake para $k=5$. os valores do NMinv dos delineamentos da região cuboidal são bem inferiores aos da região esférica. Estes delineamentos apresentam, na região cuboidal, um certo grau de robustez quanto a má especificação do modelo para valores de $k=4$ e 
coeficientes de variação em torno de $2,5 \%$.

As Tabelas 21, 22, 23 e 24 apresentam os valores da $\%$ Rep do ponto critico e NMinv dos delineamentos estudados nas regiōes esféricas e cuboidal, na situação 5 e para $4,5,6$, e 7 fatores.

Caso $k=4$. A Tabela 21 mostra que, na região esférica, todos os delineamentos apresentam valores elevados da \%Rep do ponto de sela, com os delineamentos de Hartley e Draper-Lin apresentando, também, altos valores da \%Rep do ponto de mínimo. Para $\sigma=100$, o maior valor da $\%$ Rep do ponto de máximo é apresentado pelo delineamento de Lucas. Na região cuboidal, os delineamentos de Hartley e Draper-Lin apresentam altos valores da \%Rep do ponto de sela apesar dos baixos valores do NMinv destes delineamentos. O destaque nesta região é o alto valor da \&Rep do ponto de máximo apresentado pelo delineamento de Lucas para $\sigma=100$, 0 que indica que este delineamento apresenta um certo grau de robustez quanto a má especificação do modelo.

Caso $k=5$. Na região esférica, os resultados da Tabela 22 mostram que o delineamento de Hartley apresenta um alto valor da \%Rep do ponto de mínimo. Os demais delineamentos apresentam altos valores da \%Rep do ponto de sela. Todos os delineamentos apresentam altos valores do NMinv para todos os valores de $\sigma$. Na região cuboidal, os delineamentos de Hartley, Lucas e Westlake apresentam valores mais elevados da \%Rep do ponto de máximo para $\sigma=100$. 0 NMinv apresentam uma tendência de aumento quando $\sigma$ aumenta.

Caso $k=6$. Os resultados da Tabela 23, para a região esférica, mostram que todos os delineamentos apresentam altos valores da \%Rep do ponto de sela. Na região cuboidal a tendência de altos valores da \%Rep do ponto de sela é mantido, porém com um valor de NMinv menor. Somente o 


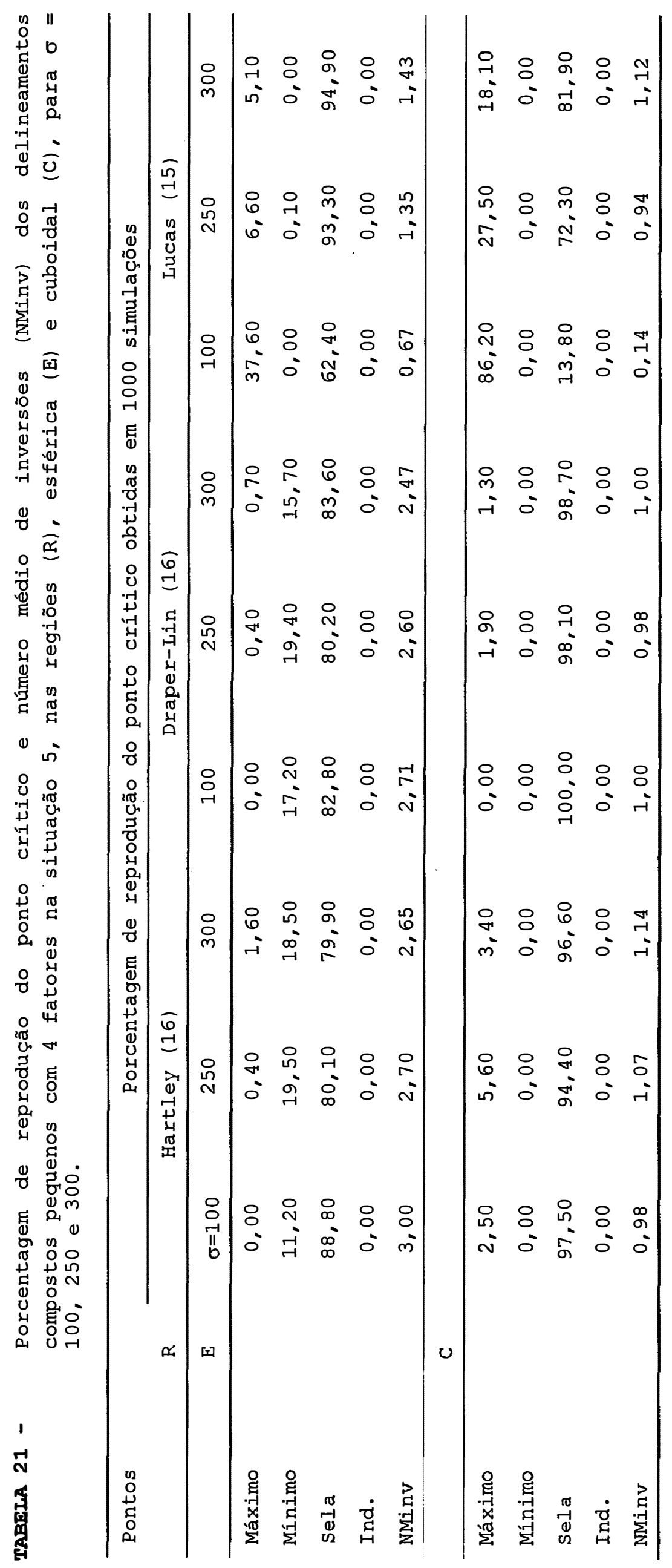




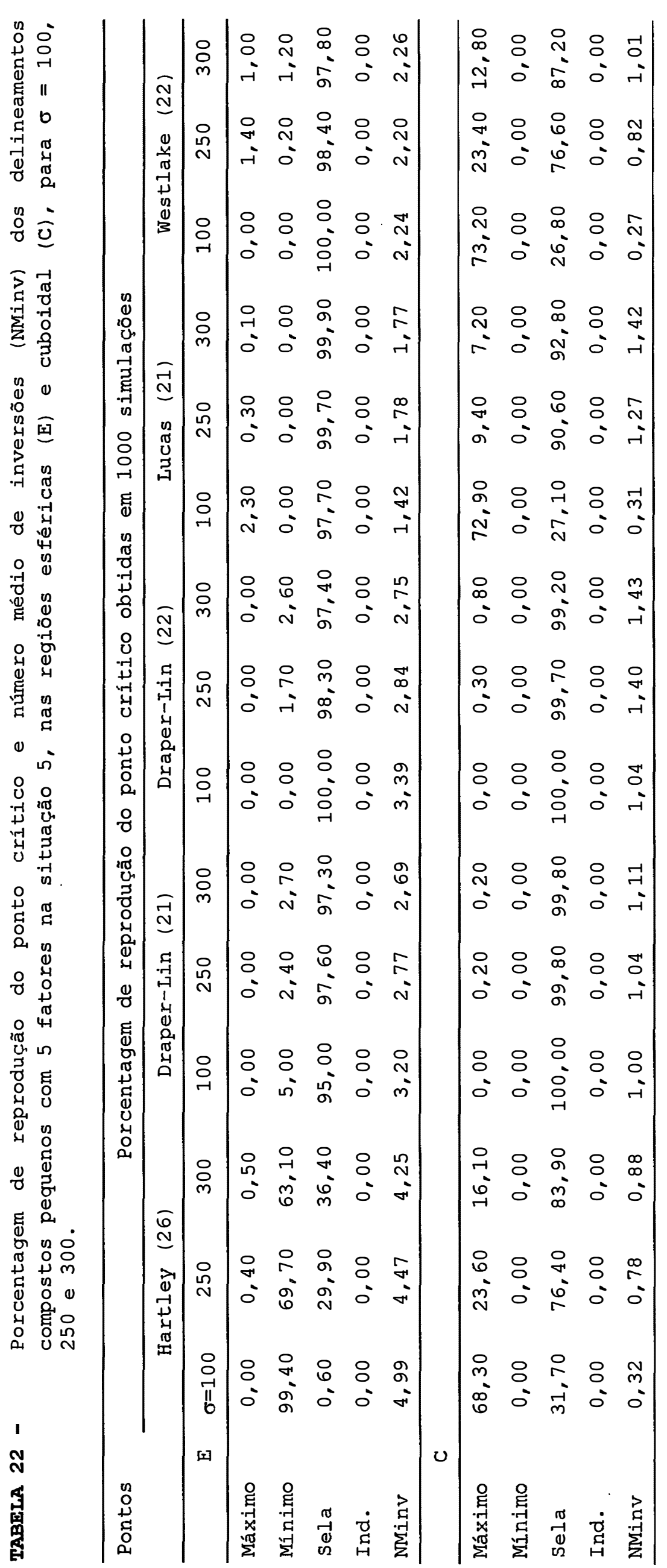




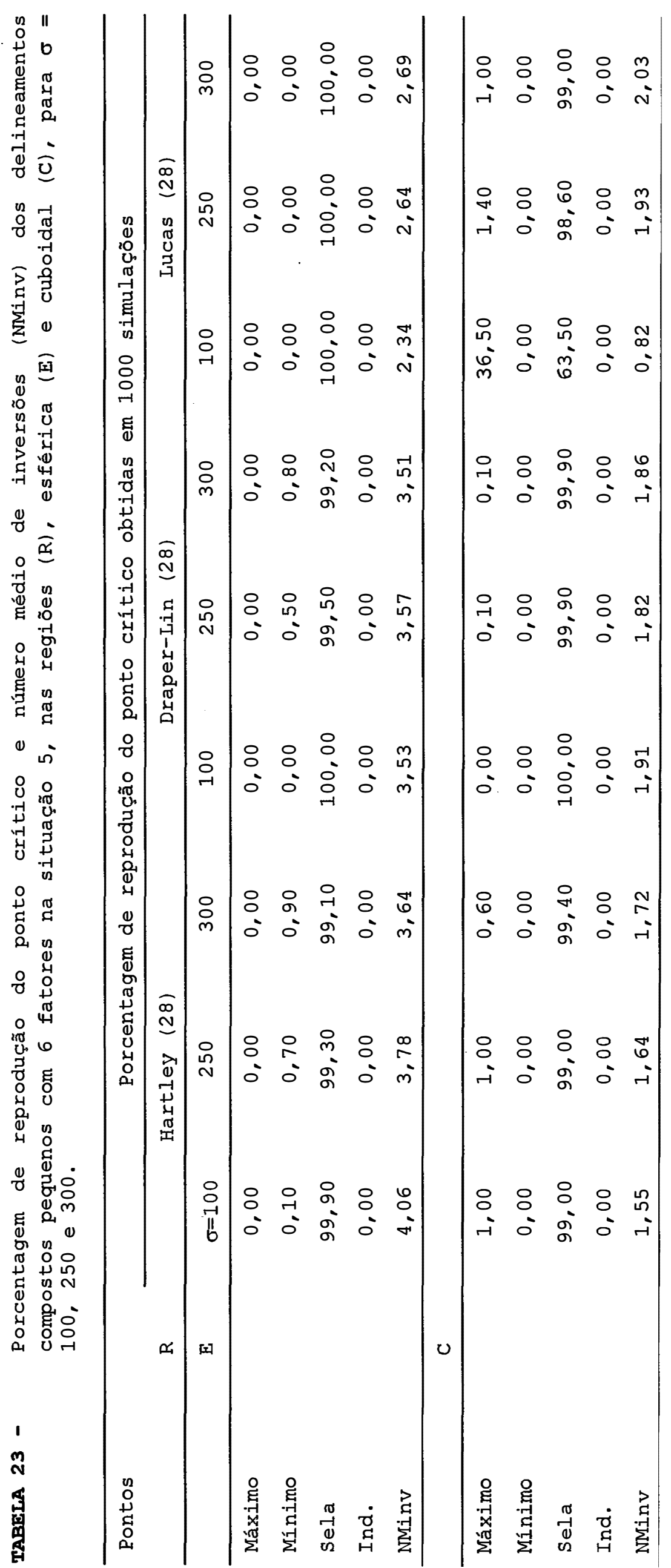




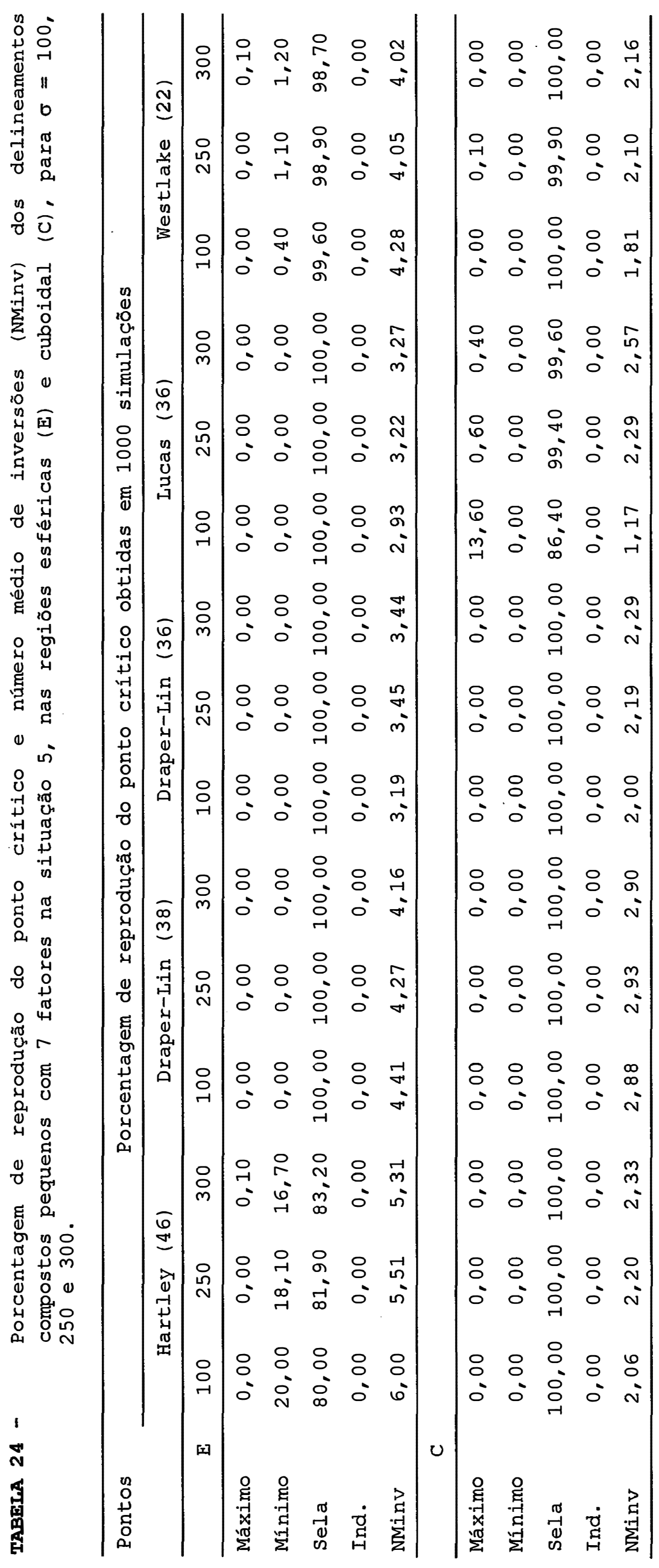


delineamento de Lucas é o que apresenta um valor da \%Rep do ponto de máximo mais elevado.

Caso $k=7$. Os resultados da Tabela 24 mostram que todos os delinamentos apresentam altos valores da $\%$ Rep do ponto de sela nas duas regiões e para os três valores de $\sigma$. O delineamento de Hartley apresenta, também, um valor expressivo da \%Rep do ponto de mínimo e um alto valor do NMinv. O valor do NMinv dos delineamentos compostos na região cuboidal é menor do que na região esférica.

A diferença nas formas entre os modelos reais e os estimados nesta situação, afeta o desempenho dos delineamentos compostos pequenos estudados neste trabalho. A fragilidade destes delineamentos fica muito evidente na região experimental esférica para qualquer valor de coeficiente de variação. o pior desempenho é 0 do delineamento de Hartley para as dimensões de $\mathrm{k}=4,5$ e 7 onde a ‡rep do ponto de mínimo é bastante elevada. O bom desempenho do delineamento de Lucas na região cuboidal, para valores de c.v. em torno $2,5 \%$ e nas dimensões $k=4$ e 5 , sugere um maior grau de robustez deste delineamento quanto a má especificação do modelo. Para $k=5$ os delineamentos de Hartley Lucas e Westlake apresentam bons resultados da \%Rep do ponto de máximo para valores do c.v. em torno de $2,5 \%$ e dimensão pequena. O NMinv da região experimental cuboidal é menor que o do região experimental esférica. 


\subsection{A medida de rotacionalidade (Q*) e os valores da distância axial $(\alpha)$ para a obtenção de arranjos em blocos ortogonais.}

Os resultados das Tabelas $25,26,27$ e 28 apresentam os valores da medida de rotacionalidade $Q^{*}$ e dos valores de $\alpha$, para vários valores de pontos centrais distribuidos na parte cubo e axial dos delineamentos compostos pequenos estudados neste trabalho, para 4, 5, 6 e 7 fatores.

Caso $k=4$. Os resultados da Tabela 25 mostram que os delineamentos de Hartley e Draper-Lin têm os mesmos valores de $\alpha$ e $Q^{*}$ em todas as combinações de pontos centrais e nos três arranjos de blocos ortogonais propostos, confirmando a equivalência destes delineamentos já verificada em itens anteriores. Os maiores valores de $Q^{*}$ foram obtidas nos seguintes arranjos: 4 pontos centrais $\left(2 n_{c 0}\right.$ no bloco I e $2 n_{s 0}$ no bloco II), 8 pontos centrais ( $2 n_{c 0}$ em cada bloco I e II, e $4 n_{s 0}$ no bloco III). O delineamento de Lucas apresenta os menores resultados de $Q^{*}$ nos vários arranjos de pontos centrais e blocos ortogonais.

Caso $k=5$. Os resultados da Tabela 26 mostram que os valores de $\alpha$ e $Q^{*}$ do delinamento de Hartley é quaserotacional para os três arranjos de blocos ortogonais propostos: 4 pontos centrais $\left(2 n_{c 0}\right.$ no bloco $I$ e $2 n_{s 0}$ no bloco II ); 6 pontos centrais ( $2 n_{c 0}$ em cada bloco I e II, e $2 n_{s 0}$ no bloco III ); 8 pontos centrais ( $2 n_{c 0}$ em cada bloco I e II, e $4 \mathrm{n}_{\mathrm{s} 0}$ no bloco III). Os valores de $Q^{*}$ dos delineamentos de Draper-Lin 22 pontos, Draper-Lin saturado e Westlake são bastante razoáveis. O delineamento de Lucas apresenta o menor valor de $Q^{*}$. 


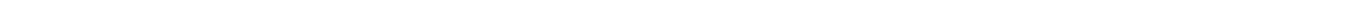


क व

$+$

d

등

ब

- $>$

ชั

in

of $>$

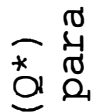

间

o

of

त्

I

-7

(0)

넌

(4)

ช

( )

응

U

न

$\rightarrow$

ช

of iो

o

c.

虫

न

ช

is

ช

नु क्षे

ह

वे

कृ

ด 0

(1) D

400

굴

$\rightarrow 0$

1

N

क्ष
ำ

몽

통

$\Phi \vec{\sigma}$

$\rightarrow$

용

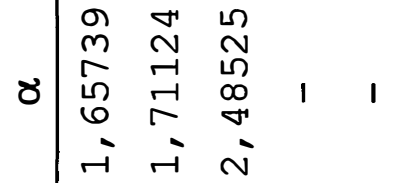

的语

要

(1) त्र

g. :

국

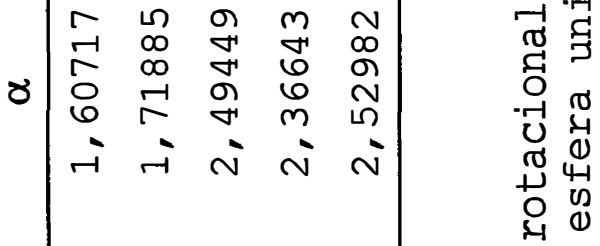

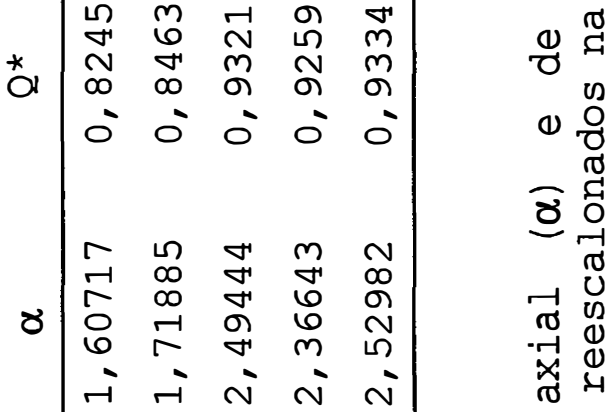

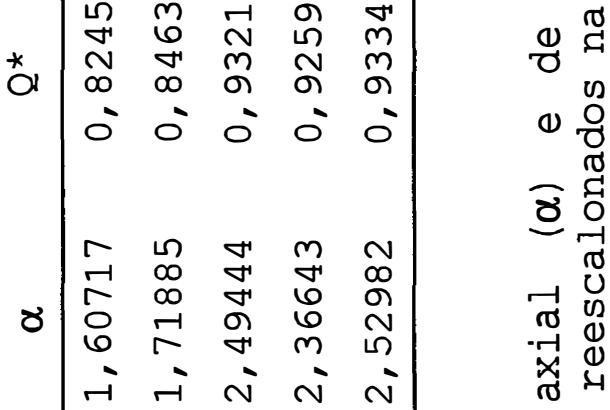

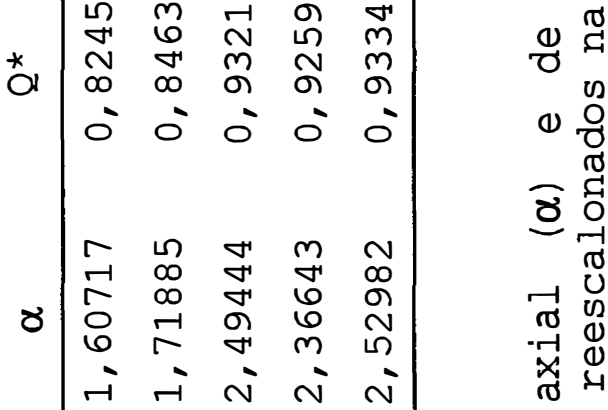

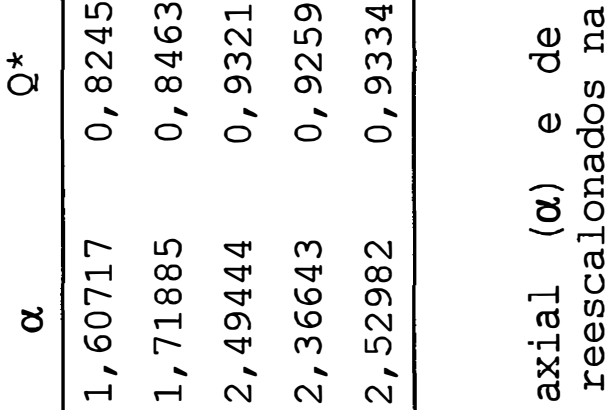

का ตे

ư

c⿷匚

to

강

ه 윰

(I)

ชั

न्तु

है छ

(1)

ช 08

थ

(1) क n

oิ

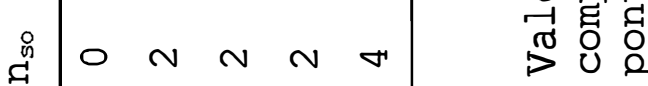

$\underset{\substack{1 \\ \sim}}{\infty}$

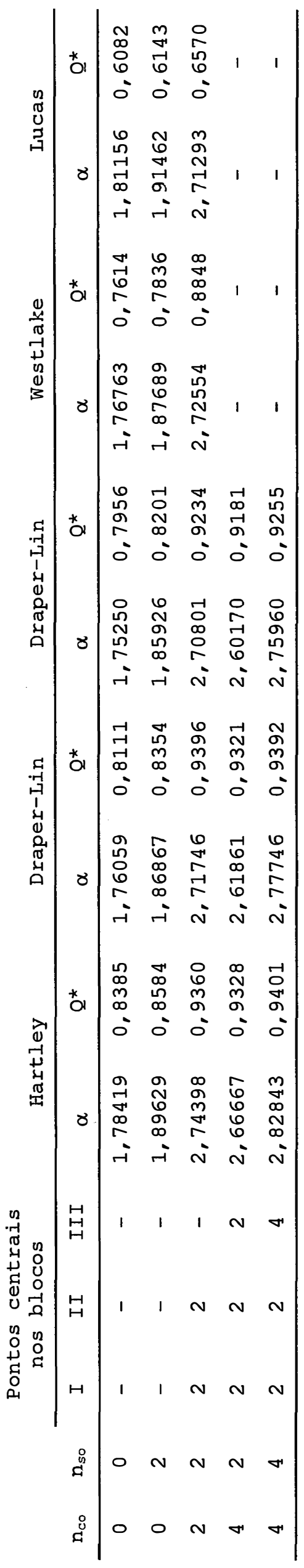


Caso $k=6$. Os dados da Tabela 27 mostram que os valores de $\alpha$ e $Q^{*}$ dos delineamentos de Hartley e Draper-Lin são iguais, confirmando a equivalência entre estes delineamentos verificada em itens anteriores. o máximo valor de $Q^{*}$ é obtido com 8 pontos centrais $\left(2 n_{c 0}\right.$ em cada bloco $I$ e II, e $4 \mathrm{n}_{\mathrm{s} 0}$ no bloco III ). O delineamento de Lucas apresenta os menores valores de $Q^{*}$.

Caso $k=7$. A Tabela 28 mostra que 0 delineamento de Hartley, apresenta o maior valor de $Q^{*}$ quando 8 pontos centrais ( $2 n_{c 0}$ em cada bloco I e II, e $4 n_{s 0}$ no bloco III ) são utilizados. Os delineamentos de Draper-Lin 22 pontos, Draper-Lin saturado e Westlake também apresentam valores interessantes de $Q^{*}$. $O$ menor valor de $Q^{*}$ é apresentado pelo delineamento de Lucas.

Observa-se destes resultados, que nenhum delineamento composto pequeno é rotacional em função dos vários valores da distância axial $(\alpha)$ e dos números de pontos centrais $n_{0}$, para os vários arranjos de blocos propostos nas Tabelas 25, 26, 27 e 28. O delineamento de Hartley para k=5 pode ser considerado como quase-rotacional. Seu valor da medida $Q^{*}=0,9968$ é bem próximo da unidade. O delineamento de Draper-Lin com 4 fatores e com 18 pontos experimentais no total, sendo 8 pontos na parte cubo, 8 pontos na parte axial e 2 pontos centrais e com $\alpha= \pm 1,68179$ é apresentado pelo software STATGRAPHIS versão 6.0, como sendo rotacional, ou seja, $Q^{*}=1$, o que não coincide com os resultados deste trabalho. A condição de $\alpha=(F)^{1 / 4}$ não é, por si só condição necessária e suficiente para que um delineamento composto seja rotacional. Ela é necessária mas não suficiente. A condição estabelecida na equação (3.7.5.5) não é satisfeita para o delineamento de Draper-Lin com 18 pontos experimentais. 
Portanto são muitas as possibilidades $e$ a flexibilidade de arranjos em blocos ortogonais dos delineamentos compostos. A primeira possibilidade que surge naturalmente é a da divisão em dois blocos: a parte cubo no bloco I e a parte axial no bloco II (veja a seção 3.7.4). Outras divisōes menores podem ser feitas se a parte cubo (fatorial ou fatorial fracionária) do delineamento composto puder ser dividida em blocos que são, por sua vez, delineamentos de primeira ordem ortogonais. A propriedade de blocos ortogonais é assegurada com a distribuição dos pontos centrais $n_{c 0}$ pelos blocos menores.

Em BOX \& DRAPER (1987), são apresentados vários exemplos de delineamentos compostos de segunda ordem úteis em arranjos de blocos ortogonais. Com base nestes exemplos, a Tabela 29 apresenta uma sugestão de arranjo de blocos ortogonais para o delineamento de Hartley com 4 fatores. O valor da distância axial $\alpha$ é determinado pela equação definida em 3.7.4.9.

o delineamento de Lucas é o que apresenta os menores valores de $Q^{*}$ em todas as dimensōes. Os delineamentos de Westlake e Lucas não apresentam possibilidades de divisão em mais de dois blocos, pois não é possível subdividir a parte cubo destes delineamentos em delineamentos ortogonais de primeira ordem. 


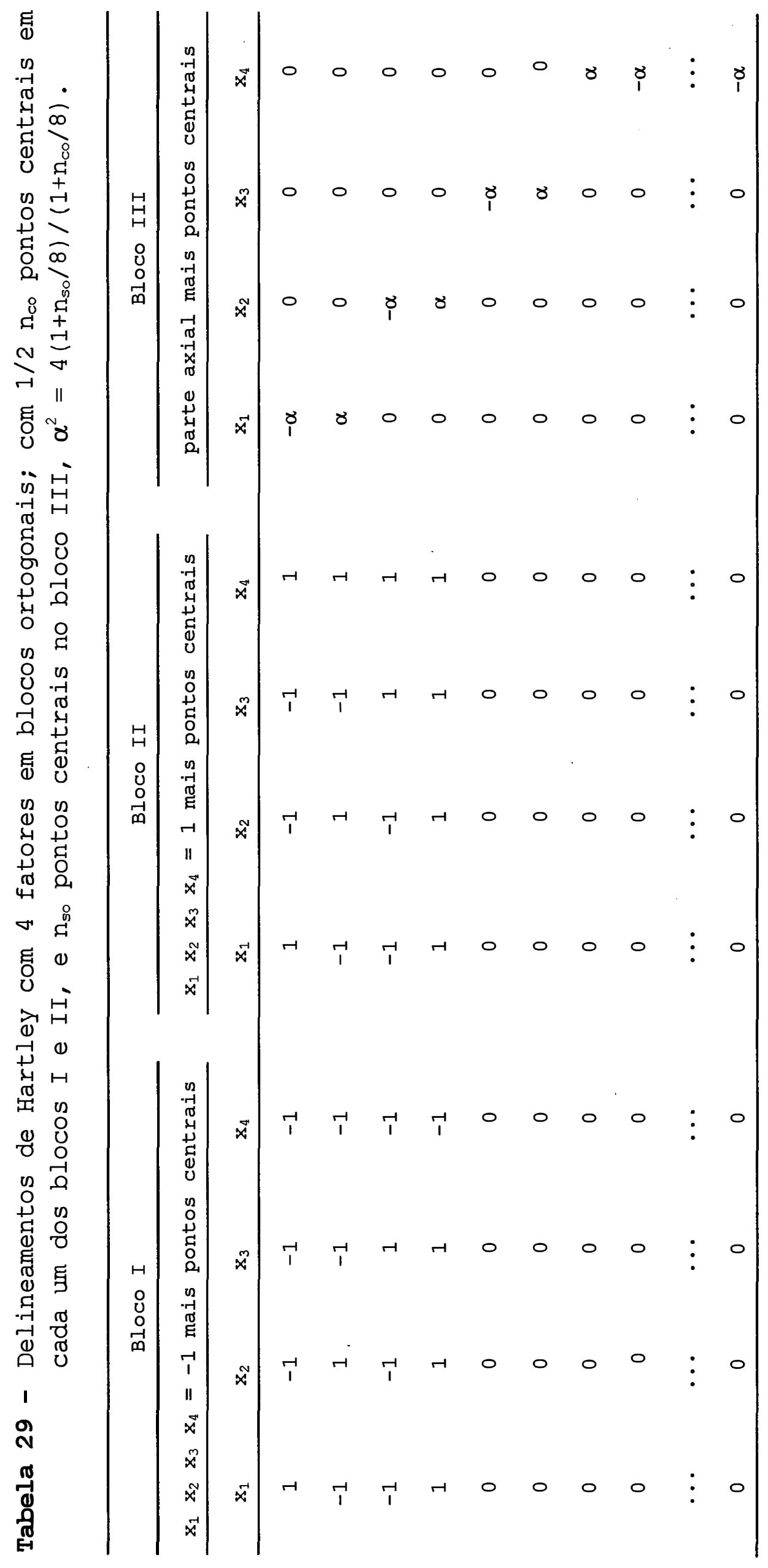




\section{CONCLUSŌES}

As conclusões e recomendações apresentadas neste trabalho, são com base nos resultados e discussões para a situação 1, na qual as formas dos modelos reais e estimados são polinomiais de segunda ordem. Nas outras situações o pesquisdador em MSR deve ter muito cuidado, pois é praticamente impossivel sugerir um delineamento para cada situação e para cada número de fatores considerados. Nestas situações, espera-se que as discussões da seção 4 estabeleçam algumas diretrizes para a tomada de decisões em MSR.

1. Para 4 fatores, na região cuboidal e pequenos valores de c.v., a recomendação é o delineamento de Hartley ou DraperLin. Como há uma equivalência entre estes delineamentos, nesta dimensão, a indicação é o delineamento de Draper-Lin pela facilidade de obtenção deste delineamento. Assim o delineamento da Tabela 5 do Apêndice, com valor de $\alpha= \pm 2$, arranjado em três blocos, com 4 pontos centrais na parte cubo e 4 pontos na parte axial é o mais indicado para este caso. Na região cuboidal, a sugestão, também é o delineamento de Draper-Lin (Tabela 5 do Apêndice), arranjado em três blocos, com 4 pontos centrais na parte cubo e 4 pontos na parte axial $e, \alpha$ assumindo os valores 0 e 5 , ou seja centrados na face do hiper-cubo de dimensão 4. Estes delineamentos têm o menor tamanho para experimentos que envolvem 4 fatores e são quaserotacionais.

2. Para 5 fatores, na região esférica e com valores de coeficientes de variação em torno de $2,5 \%(\sigma=100)$, o 
delineamento de Hartley, descrito na Tabela 2 do Apêndice, é o indicado. A distância axial pode ser $\alpha= \pm 2,2360$, arranjado em três blocos e com 4 pontos centrais na parte cubo e 4 pontos centrais na parte axial. Neste arranjo o delineamento apresenta uma medida de rotacionalidade de $Q^{*}=0,9968$, o que pode ser considerado como quase-rotacional. Na região cuboidal os delineamentos de Hartley, Draper-Lin (22 pontos) e Westlake apresentam excelentes resultados para os três valores de coeficientes de variação estudados. A recomendação é o delineamento de Draper-Lin (22 pontos), Tabela 7 do Apêndice, pela sua facilidade de obtenção e por ser quasesaturado, tem apenas 1 ponto experimental a mais que o número de parâmetros do modelo de segunda ordem para 5 fatores. Este delineamento pode ser arranjado em três blocos com 4 pontos centrais na parte cubo e 4 pontos centrais na parte axial do delineamento, com $\alpha$ assumindo os valores 0 e 5 , ou seja, no centro de cada face do hiper-cubo de dimensão 5 .

3. Para 6 fatores, na região esférica e para valores de c.v. pequenos, os delineamentos de Hartley e Draper-Lin são indicados. Devido a equivalência destes delineamentos a indicação é o delineamento de Draper-Lin, Tabela 8 do Apêndice, arranjados em três blocos, com $\alpha= \pm 2,52982$, com 4 pontos centrais na parte cubo e 4 pontos centrais na parte axial, este delineamento é quase-rotacional com $Q^{*}=0,9334$. Na região cuboidal, novamente o delineamento de Draper-Lin é o indicado, arranjado em três blocos, com 4 pontos centrais na parte cubo e 4 pontos na parte axial e $\alpha$ assumindo os valores o e 5,ou seja, centrados na face do hiper-cubo de dimensão 6 e para os três valores do coeficiente de variação.

4. Para 7 fatores, na região esférica e para um coeficiente de variação pequeno a indicação é o delineamento de Hartley, Tabela 4 do Apêndice. Este delineamento arranjado em três blocos, com $\alpha= \pm 2,82843$, com 4 pontos centrais na parte cubo 
e 4 pontos centrais na parte axial, apresenta uma medida de rotacionalidade $Q^{*}=0,9401$. Na região cuboidal o delineamento de Hartley também é o sugerido para os três valores de coeficientes de variação considerados neste trabalho. Este delineamento pode ser arranjado em três blocos, com 4 pontos centrais na parte cubo e 4 pontos centrais na parte axial e, $\alpha$ assumindo os valores 0 e 5 . 


\section{REFERÊNCIAS BIBLIOGRÁFICAS}

ANDRADE, D.F., NOLETO, A.Q. Exemplos de fatoriais fracionados $(1 / 2) 4^{3}$ e $(1 / 4) 4^{4}$ para 0 ajuste de modelos polinomiais quadráticos. In: SIMPÓsIo DE ESTATÍsTICA APLICADA À EXPERIMENTAÇÃo AGRONÔMICA, 1, 1985, Piracicaba. Anais... Campinas: Fundação Cargill, 1985. p.183-196.

ATCKINSON, A.C., DONEV, A.N. Optimum experimental designs. New York: Oxford University Press, 1992. 328p.

ATWOOD, C.L. Optimal and efficient designs of experiments. Ann. Math. Statist. v.40, p.1570-1602, 1969.

BOX, G.E.P. Experimental design; response surface methodology. In: KURNSKAL W.R., TARNUR, J.M. (Eds.) • Internacional Encyclopedia of statistics, 1 . New York: Free Press, 1978. p.294-299.

BOX, G.E.P. Choice of response surface design and alphabetic optimality. Utilitas Mathematica, v.21, p.11-15, 1982.

BOX, G.E.P., BEHNKEN, D.W. Some new three-level designs for the study of quantitative variables. Technometrics, v.2, p.455-475, 1960 .

BOX, G.E.P., DRAPER, N.R. A basis for the selection of a response surface design. J. Amer. statist. Ass.. V.54, p.622-654, 1959 .

BOX, G.E.P., DRAPER, N.R. The choice of a second order rotatable design. Biometrika, v.50, p.335-352, 1963. 
BOX, G.E.P., DRAPER, N.R. Robust designs. Biometrika, V.62, p.347-352, 1975 .

BOX, G.E.P., DRAPER, N.R. Empirical model building and response surfaces. New York: John Wiley, 1987. 669p.

BOX, G.E.P., HUNTER, J.S. Multifactor experimental designs for exploring response surfaces. Ann. Math. statist. v.28, p.195-241, 1957.

BOX, G.E.P., HUNTER, J.S. The $2^{k-p}$ fractional factorial designs. Part I. Technometrics, v.3, p.311-351, 1961a.

BOX, G.E.P., HUNTER, J.S. The $2^{\text {k-p }}$ fractional factorial designs. Technometrics, v.3, p.449-458, 1961b.

BOX, G.E.P., HUNTER, W.G., HUNTER, J.S. statistics for experimenters. New York: John Wiley, 1978.

BOX, G.E.P. \& MULLER, M.E. A note on the generation of randon normal deviates. Ann. Math. Statist., 29: 610-611, 1958.

BOX, G.E.P., WILSON, K.B. On the experimental attainment of optimum conditions. J.R. statist. Soc.. B, v.13, p.1-45, 1951.

CONAGIN, A., JORGE, J.P.N. Delineamentos (1/5) $5^{3}$. Bragantia, v.36, p.23-58, 1977 .

CROWTHER, E.M., YATES, J.F. Fertiliser policy in war-time. Empire J. Exp. Agric., v.9, p.77-97, 1941.

DAVIES, O.L. The design and analysis of industrial 
experiments. New York: Hafner Press, 1954.

De BAUN, R.M. Response surface designs for three factors at three levels. Technometrics, v.1, p.1-8, 1959.

DRAPER, N.R. Small composite designs. Technometrics, v.27, p.173-180, 1985 .

DRAPER, N.R. An index of rotatability. Technometrics, v.30, p.105-111, 1988 .

DRAPER, N.R., GUTTMAN, I. An index of rotatability. Technometrics, v.30, p.105-111, 1988 .

DRAPER, N.R., LIN, D.K.J. Small response-surface designs. Technometrics, v.32, p.187-194, 1990 .

DRAPER, N.R., PUKELSHEIM, F. Another look at rotatability. Technometrics, v.32, p.195-202, 1990 .

EDMONDSON, R.N. Agricultural response surface experiment based on four-level factorial designs. Biometrics, v.47, p.1435-1448, 1991 .

FEDOROV, V.V. Theory of optimal experiments. New York: Academic Press, 1972. 292p.

HARTLEY, H.D. Smallest composite design for quadratic response surface. Biometrics, v.15, p.611-624, 1959.

HEXEM, R.W. \& HEADY, E.O. Water production functions for irrigated agriculture. Ames: The Iowa state University Press. $215 \mathrm{p}$. 
HILL, W.J., HUNTER, W.G. A review of response surface methodology: A literature review. Technometrics, v.8, p.571-590, 1966 .

KHURI, A.T. A measure of rotatability of response-surface design. Technometrics, v.21, p.467-473, 1988.

KHURI, A.T., CORNELL, J. A. Response surfaces. New York: Marcel Dekker, Inc., 1987. 405p.

KIEFER, J. On the nonrandomized optimality and the randomized nonoptimality of symmetrical designs, Ann. Math. statist., v.29, p.675-699, 1958.

KIEFER, J. Optimum experimental designs (with discussion). J. R. statist. Soc., v.21, p.272-319, 1959 .

KIEFER, J. Optimum experimental designs $V$, with applications to systematic and rotatable designs. In: Proceedings of the Fourth Berkeley Symposium on Mathematical statistics and Probability, 1, 1960. Berkeley. Anais... Berkeley: University of California Press, 1960. p.381-405.

KIEFER, J. Optimum designs in regression problems II. Ann. Math. Statist., v.32, p.298-325, 1961.

KIEFER, J. An extremum result. Canadiam J. Math., v.14, p.597-601. 1962a.

KIEFER, J. Two more criteria equivalent to D-optimality of designs. Ann. Math. Statist., v.33, p.792-796. 1962 b.

KIEFER, J., WOLFOWITZ, J. Optimum designs in regression 
problems. Ann. Math. Statist., v.30, p.271-294, 1959.

KIEFER, J., WOLFOWITZ, J. The equivalence of two extremum problems. Canadiam J. Math., v.12, p.363-366, 1960.

LIN, D. K. J. A new class of supersatureted designs. Technometrics, v. 35, p.28-31, 1993

LUCAS, J.M. Optimum composite design. Technometrics, v.16, p.561-567, 1974 .

MEAD, R. The non-ortogonal designs of experiments. $J$. $\mathbf{R}$. statist. Soc.. A, v.153, Part 2, p.151-201, 1990.

MEAD, R., PIKE, D.J. A review of response surface methodology from a biometrics viewpoint. Biometrics, v.31, p. 803-851, 1975 .

MITCHELL, T.J. An algorithm for the construction of D-optimal experimental designs. Technometrics, v.16, p.203-210, 1974 .

MYERS, R.H. Response surface methodology • Blacksburg, V.A:Author. 1976. 246p.

MYERS, R.H., KHURI, A.I., CARTER Jr., W.H. Response surface methodology: 1966-1988. Technometrics, v.31, n.22, p.137157 , 1989.

NALIMOV, V.V., GOLIKOVA, T.I., MIKESHIMA, N.G. On pratical use of the concept of D-otimality. Technometrics, v.12, p.799-812, 1970 . 
PERECIN, D., MALHEIROS, E.B., BANZATTO, D.A. Tipos de delineamentos $(1 / 5) 5^{3}$ e superfície de respostas. cientifica, v.10, p.193-201, 1982 .

PEREIRA, G.T. Partiçōes e eficiência com delineamentos $(1 / 5) 5^{3}$ e superficie de resposta. Ribeirão Preto, 1984. 100p. (M.S - Universidade de São Paulo)

PEREIRA, G.T., PERECIN, D. Eficiência de delineamentos $(1 / 5) 5^{3}$, em relação ao delineamento composto central ortogonal (15 pontos). In: SIMPÓSIO DE ESTATÍSTICA APLICADA À EXPERIMENTAÇÃO AGRONÔMICA, 1, 1985, Piracicaba. Anais... Campinas: Fundação Cargill, 1985. p.183-196.

PEREIRA, G.T., PERECIN, D. Eficiência de delineamentos $(1 / 5) 5^{3}$. Rev. Mat. Estat., V.8, p.125-134, 1990.

PLACKETT, R.L., BURMAN, J.P. The design of multifactorial experiments. Biometrika, v.33, p.305-325, 1946.

ROQUEMORE, K.G. Hybrid designs for quadratic response surfaces. Technometrics, v.18, p.419-423, 1976.

SEARLE, S.R. Matrix algebra useful for statistics. New York: John Wiley \& Sons. 1982. 438p.

SANCHES, A. Construção de modelos matemáticos para simulação de dados de produção em experimentos de adubação. Rev. Mat. Estat., v.9, p.125-135, 1991.

SANCHES, A. Superficies de resposta em experimentos de adubação: o problema dos pontos de sela. Piracicaba, 1986. 91 p. (Doutorado - Escola Superior de Agricultura "Luiz de 
Queiroz"/USP) .

WESTLAKE, W.J. Composite designs based on irregular fractions of factorials. Biometrics, v.21, p.324-336, 1965 .

YATES, F. Complex experiments (with discussion). J.R. statist. soc.. B, v.34, p.133-147, 1935 . 
APÊNDICE 
Tabela 1 - Delineamento composto quase saturado de Hartley para 4 fatores $\left(\mathrm{Ha}_{4}\right)$

\begin{tabular}{rrrrrrrrrr}
\hline $\mathrm{N} 2$ & $\mathrm{X}_{1}$ & $\mathrm{X}_{2}$ & $\mathrm{X}_{3}$ & $\mathrm{X}_{4}$ & $\mathrm{~N} 0$ & $\mathrm{X}_{1}$ & $\mathrm{X}_{2}$ & $\mathrm{X}_{3}$ & $\mathrm{X}_{4}$ \\
\hline 1 & 1 & -1 & -1 & -1 & 9 & $-\alpha$ & 0 & 0 & 0 \\
2 & -1 & 1 & -1 & -1 & 10 & $\alpha$ & 0 & 0 & 0 \\
3 & -1 & -1 & 1 & -1 & 11 & 0 & $-\alpha$ & 0 & 0 \\
4 & 1 & 1 & 1 & -1 & 12 & 0 & $\alpha$ & 0 & 0 \\
5 & 1 & -1 & -1 & 1 & 13 & 0 & 0 & $-\alpha$ & 0 \\
6 & -1 & 1 & -1 & 1 & 14 & 0 & 0 & $\alpha$ & 0 \\
7 & -1 & -1 & 1 & 1 & 15 & 0 & 0 & 0 & $-\alpha$ \\
8 & 1 & 1 & 1 & 1 & 16 & 0 & 0 & 0 & $\alpha$ \\
\hline
\end{tabular}


Tabela 2 - Delineamento composto quase saturado de Hartley para cinco fatores $\left(\mathrm{Ha}_{5}\right)$.

\begin{tabular}{lrrrrrrrrrrr}
\hline $\mathrm{N} 2$ & $\mathrm{X}_{1}$ & $\mathrm{X}_{2}$ & $\mathrm{X}_{3}$ & $\mathrm{X}_{4}$ & $\mathrm{X}_{5}$ & $\mathrm{~N} 0$ & $\mathrm{X}_{1}$ & $\mathrm{X}_{2}$ & $\mathrm{X}_{3}$ & $\mathrm{X}_{4}$ & $\mathrm{X}_{5}$ \\
\hline 1 & 1 & -1 & -1 & -1 & -1 & 14 & -1 & 1 & -1 & 1 & 1 \\
2 & -1 & 1 & -1 & -1 & -1 & 15 & -1 & -1 & 1 & 1 & 1 \\
3 & -1 & -1 & 1 & -1 & -1 & 16 & 1 & 1 & 1 & 1 & 1 \\
4 & 1 & 1 & 1 & -1 & -1 & 17 & $-\alpha$ & 0 & 0 & 0 & 0 \\
5 & -1 & -1 & -1 & 1 & -1 & 18 & $\alpha$ & 0 & 0 & 0 & 0 \\
6 & 1 & 1 & -1 & 1 & -1 & 19 & 0 & $-\alpha$ & 0 & 0 & 0 \\
7 & 1 & -1 & 1 & 1 & -1 & 20 & 0 & $\alpha$ & 0 & 0 & 0 \\
8 & -1 & 1 & 1 & 1 & -1 & 21 & 0 & 0 & $-\alpha$ & 0 & 0 \\
9 & -1 & -1 & -1 & -1 & 1 & 22 & 0 & 0 & $\alpha$ & 0 & 0 \\
10 & 1 & 1 & -1 & -1 & 1 & 23 & 0 & 0 & 0 & $-\alpha$ & 0 \\
11 & 1 & -1 & 1 & -1 & 1 & 24 & 0 & 0 & 0 & $\alpha$ & 0 \\
12 & -1 & 1 & 1 & -1 & 1 & 25 & 0 & 0 & 0 & 0 & $-\alpha$ \\
13 & 1 & -1 & -1 & 1 & 1 & 26 & 0 & 0 & 0 & 0 & $\alpha$ \\
\hline
\end{tabular}


Tabela 3 - Delineamento composto quase saturado de Hartley para seis fatores $\left(\mathrm{Ha}_{6}\right)$.

\begin{tabular}{lrrrrrrrrrrrrr}
\hline $\mathrm{N} 0$ & $\mathrm{X}_{1}$ & $\mathrm{X}_{2}$ & $\mathrm{X}_{3}$ & $\mathrm{X}_{4}$ & $\mathrm{X}_{5}$ & $\mathrm{X}_{6}$ & $\mathrm{~N} 0$ & $\mathrm{X}_{1}$ & $\mathrm{X}_{2}$ & $\mathrm{X}_{3}$ & $\mathrm{X}_{4}$ & $\mathrm{X}_{5}$ & $\mathrm{X}_{6}$ \\
\hline 1 & 1 & -1 & -1 & 1 & -1 & -1 & 15 & -1 & -1 & 1 & 1 & 1 & 1 \\
2 & -1 & 1 & -1 & 1 & -1 & -1 & 16 & 1 & 1 & 1 & 1 & 1 & 1 \\
3 & -1 & -1 & 1 & 1 & -1 & -1 & 17 & $-\alpha$ & 0 & 0 & 1 & 0 & 0 \\
4 & 1 & 1 & 1 & 1 & -1 & -1 & 18 & $\alpha$ & 0 & 0 & 0 & 0 & 0 \\
5 & 1 & -1 & -1 & -1 & 1 & -1 & 19 & 0 & $-\alpha$ & 0 & 0 & 0 & 0 \\
6 & -1 & 1 & -1 & -1 & 1 & -1 & 20 & 0 & $\alpha$ & 0 & 0 & 0 & 0 \\
7 & -1 & -1 & 1 & -1 & 1 & -1 & 21 & 0 & 0 & $-\alpha$ & 0 & 0 & 0 \\
8 & 1 & 1 & 1 & -1 & 1 & -1 & 22 & 0 & 0 & $\alpha$ & 0 & 0 & 0 \\
9 & 1 & -1 & -1 & -1 & -1 & 1 & 23 & 0 & 0 & 0 & $-\alpha$ & 0 & 0 \\
10 & -1 & 1 & -1 & -1 & -1 & 1 & 24 & 0 & 0 & 0 & $\alpha$ & 0 & 0 \\
11 & -1 & -1 & 1 & -1 & -1 & 1 & 25 & 0 & 0 & 0 & 0 & $-\alpha$ & 0 \\
12 & 1 & 1 & 1 & -1 & -1 & 1 & 26 & 0 & 0 & 0 & 0 & $\alpha$ & 0 \\
13 & 1 & -1 & -1 & 1 & 1 & 1 & 27 & 0 & 0 & 0 & 0 & 0 & $-\alpha$ \\
14 & -1 & 1 & -1 & 1 & 1 & 1 & 28 & 0 & 0 & 0 & 0 & 0 & $\alpha$ \\
\hline & & & & & & & & & & & &
\end{tabular}


Tabela 4 - Delineamento composto quase saturado de Hartley para sete fatores $\left(\mathrm{Ha}_{7}\right)$.

\begin{tabular}{lrrrrrrrrrrrrrrr}
\hline $\mathrm{N} 2$ & $\mathrm{X}_{1}$ & $\mathrm{X}_{2}$ & $\mathrm{X}_{3}$ & $\mathrm{X}_{4}$ & $\mathrm{X}_{5}$ & $\mathrm{X}_{6}$ & $\mathrm{X}_{7}$ & $\mathrm{~N} 0$ & $\mathrm{X}_{1}$ & $\mathrm{X}_{2}$ & $\mathrm{X}_{3}$ & $\mathrm{X}_{4}$ & $\mathrm{X}_{5}$ & $\mathrm{X}_{6}$ & $\mathrm{X}_{7}$ \\
\hline 1 & 1 & -1 & -1 & 1 & -1 & -1 & -1 & 24 & 1 & 1 & 1 & -1 & 1 & -1 & 1 \\
2 & -1 & 1 & -1 & 1 & -1 & -1 & -1 & 25 & 1 & -1 & -1 & -1 & -1 & 1 & 1 \\
3 & -1 & -1 & 1 & 1 & -1 & -1 & -1 & 26 & -1 & 1 & -1 & -1 & -1 & 1 & 1 \\
4 & 1 & 1 & 1 & 1 & -1 & -1 & -1 & 27 & -1 & -1 & 1 & -1 & -1 & 1 & 1 \\
5 & 1 & -1 & -1 & -1 & 1 & -1 & -1 & 28 & 1 & 1 & 1 & -1 & -1 & 1 & 1 \\
6 & -1 & 1 & -1 & -1 & 1 & -1 & -1 & 29 & 1 & -1 & -1 & 1 & 1 & 1 & 1 \\
7 & -1 & -1 & 1 & -1 & 1 & -1 & -1 & 30 & -1 & 1 & -1 & 1 & 1 & 1 & 1 \\
8 & 1 & 1 & 1 & -1 & 1 & -1 & -1 & 31 & -1 & -1 & 1 & 1 & 1 & 1 & 1 \\
9 & 1 & -1 & -1 & -1 & -1 & 1 & -1 & 32 & 1 & 1 & 1 & 1 & 1 & 1 & 1 \\
10 & -1 & 1 & -1 & -1 & -1 & 1 & -1 & 33 & $-\alpha$ & 0 & 0 & 0 & 0 & 0 & 0 \\
11 & -1 & -1 & 1 & -1 & -1 & 1 & -1 & 34 & $\alpha$ & 0 & 0 & 0 & 0 & 0 & 0 \\
12 & 1 & 1 & 1 & -1 & -1 & 1 & -1 & 35 & 0 & $-\alpha$ & 0 & 0 & 0 & 0 & 0 \\
13 & 1 & -1 & -1 & 1 & 1 & 1 & -1 & 36 & 0 & $\alpha$ & 0 & 0 & 0 & 0 & 0 \\
14 & -1 & 1 & -1 & 1 & 1 & 1 & -1 & 37 & 0 & 0 & $-\alpha$ & 0 & 0 & 0 & 0 \\
15 & -1 & -1 & 1 & 1 & 1 & 1 & -1 & 38 & 0 & 0 & $\alpha$ & 0 & 0 & 0 & 0 \\
16 & 1 & 1 & 1 & 1 & 1 & 1 & -1 & 39 & 0 & 0 & 0 & $-\alpha$ & 0 & 0 & 0 \\
17 & 1 & -1 & -1 & 1 & -1 & -1 & 1 & 40 & 0 & 0 & 0 & $\alpha$ & 0 & 0 & 0 \\
18 & -1 & 1 & -1 & 1 & -1 & -1 & 1 & 41 & 0 & 0 & 0 & 0 & $-\alpha$ & 0 & 0 \\
19 & -1 & -1 & 1 & 1 & -1 & -1 & 1 & 42 & 0 & 0 & 0 & 0 & $\alpha$ & 0 & 0 \\
20 & 1 & 1 & 1 & 1 & -1 & -1 & 1 & 43 & 0 & 0 & 0 & 0 & 0 & $-\alpha$ & 0 \\
21 & 1 & -1 & -1 & -1 & 1 & -1 & 1 & 44 & 0 & 0 & 0 & 0 & 0 & $\alpha$ & 0 \\
22 & -1 & 1 & -1 & -1 & 1 & -1 & 1 & 45 & 0 & 0 & 0 & 0 & 0 & 0 & $-\alpha$ \\
23 & -1 & -1 & 1 & -1 & 1 & -1 & 1 & 46 & 0 & 0 & 0 & 0 & 0 & 0 & $\alpha$ \\
\hline & & & & & & & & & & & & & &
\end{tabular}


Tabela 5 - Delineamento composto quase saturado de Draper-Lin para quatro fatores $\left(\mathrm{DL}_{4}\right)$.

\begin{tabular}{lrrrrrrrrr}
\hline $\mathrm{N} 0$ & $\mathrm{X}_{1}$ & $\mathrm{X}_{2}$ & $\mathrm{X}_{3}$ & $\mathrm{X}_{4}$ & $\mathrm{~N} 0$ & $\mathrm{X}_{1}$ & $\mathrm{x}_{2}$ & $\mathrm{X}_{3}$ & $\mathrm{x}_{4}$ \\
\hline 1 & 1 & 1 & 1 & -1 & 9 & $-\alpha$ & 0 & 0 & 0 \\
2 & 1 & 1 & -1 & -1 & 10 & $\alpha$ & 0 & 0 & 0 \\
3 & 1 & -1 & 1 & 1 & 11 & 0 & $-\alpha$ & 0 & 0 \\
4 & -1 & 1 & -1 & 1 & 12 & 0 & $\alpha$ & 0 & 0 \\
5 & 1 & -1 & -1 & 1 & 13 & 0 & 0 & $-\alpha$ & 0 \\
6 & -1 & -1 & 1 & -1 & 14 & 0 & 0 & $\alpha$ & 0 \\
7 & -1 & 1 & 1 & 1 & 15 & 0 & 0 & 0 & $-\alpha$ \\
8 & -1 & -1 & -1 & -1 & 16 & 0 & 0 & 0 & $\alpha$ \\
\hline
\end{tabular}


Tabela 6 - Delineamento composto saturado de Draper-Lin para cinco fatores $\left(\mathrm{DL}_{5}\right)$.

\begin{tabular}{lrrrrrrrrrrr}
\hline $\mathrm{N} 9$ & $\mathrm{X}_{1}$ & $\mathrm{X}_{2}$ & $\mathrm{X}_{3}$ & $\mathrm{X}_{4}$ & $\mathrm{X}_{5}$ & $\mathrm{~N} 0$ & $\mathrm{X}_{1}$ & $\mathrm{X}_{2}$ & $\mathrm{X}_{3}$ & $\mathrm{X}_{4}$ & $\mathrm{X}_{5}$ \\
\hline 1 & 1 & 1 & -1 & 1 & -1 & 12 & $-\alpha$ & 0 & 0 & 0 & 0 \\
2 & 1 & -1 & 1 & 1 & -1 & 13 & $\alpha$ & 0 & 0 & 0 & 0 \\
3 & -1 & 1 & 1 & -1 & 1 & 14 & 0 & $-\alpha$ & 0 & 0 & 0 \\
4 & 1 & 1 & 1 & -1 & -1 & 15 & 0 & $\alpha$ & 0 & 0 & 0 \\
5 & 1 & 1 & -1 & -1 & 1 & 16 & 0 & 0 & $-\alpha$ & 0 & 0 \\
6 & 1 & -1 & -1 & 1 & 1 & 17 & 0 & 0 & $\alpha$ & 0 & 0 \\
7 & -1 & -1 & 1 & 1 & 1 & 18 & 0 & 0 & 0 & $-\alpha$ & 0 \\
8 & -1 & 1 & 1 & 1 & 1 & 19 & 0 & 0 & 0 & $\alpha$ & 0 \\
9 & 1 & -1 & -1 & -1 & 1 & 20 & 0 & 0 & 0 & 0 & $-\alpha$ \\
10 & -1 & 1 & 1 & 1 & -1 & 21 & 0 & 0 & 0 & 0 & $\alpha$ \\
11 & -1 & -1 & -1 & -1 & -1 & & & & & & \\
\hline
\end{tabular}


Tabela 7 - Delineamento composto quase saturado de DraperLin para cinco fatores $\left(\mathrm{DL}_{5}\right)$.

\begin{tabular}{lrrrrrrrrrrr}
\hline $\mathrm{N} 2$ & $\mathrm{X}_{1}$ & $\mathrm{X}_{2}$ & $\mathrm{X}_{3}$ & $\mathrm{X}_{4}$ & $\mathrm{X}_{5}$ & $\mathrm{~N} 0$ & $\mathrm{X}_{1}$ & $\mathrm{X}_{2}$ & $\mathrm{X}_{3}$ & $\mathrm{X}_{4}$ & $\mathrm{X}_{5}$ \\
\hline 1 & 1 & 1 & -1 & 1 & 1 & 12 & -1 & -1 & -1 & -1 & -1 \\
2 & 1 & -1 & 1 & 1 & 1 & 13 & $-\alpha$ & 0 & 0 & 0 & 0 \\
3 & -1 & 1 & 1 & 1 & -1 & 14 & $\alpha$ & 0 & 0 & 0 & 0 \\
4 & 1 & 1 & 1 & -1 & -1 & 15 & 0 & $-\alpha$ & 0 & 0 & 0 \\
5 & 1 & 1 & -1 & -1 & -1 & 16 & 0 & $\alpha$ & 0 & 0 & 0 \\
6 & 1 & -1 & -1 & -1 & 1 & 17 & 0 & 0 & $-\alpha$ & 0 & 0 \\
7 & -1 & -1 & 1 & 1 & -1 & 18 & 0 & 0 & $\alpha$ & 0 & 0 \\
8 & -1 & -1 & -1 & -1 & 1 & 19 & 0 & 0 & 0 & $-\alpha$ & 0 \\
9 & -1 & 1 & 1 & 1 & 1 & 20 & 0 & 0 & 0 & $\alpha$ & 0 \\
10 & 1 & -1 & 1 & 1 & -1 & 21 & 0 & 0 & 0 & 0 & $-\alpha$ \\
11 & -1 & 1 & -1 & -1 & 1 & 22 & 0 & 0 & 0 & 0 & $\alpha$ \\
\hline
\end{tabular}


Tabela 8 - Delineamento composto saturado de Draper-Iin para seis fatores $\left(D_{6}\right)$.

\begin{tabular}{lrrrrrrrrrrrrr}
\hline $\mathrm{N} 0$ & $\mathrm{X}_{1}$ & $\mathrm{X}_{2}$ & $\mathrm{X}_{3}$ & $\mathrm{X}_{4}$ & $\mathrm{X}_{5}$ & $\mathrm{X}_{6}$ & $\mathrm{~N} 0$ & $\mathrm{X}_{1}$ & $\mathrm{X}_{2}$ & $\mathrm{X}_{3}$ & $\mathrm{X}_{4}$ & $\mathrm{X}_{5}$ & $\mathrm{X}_{6}$ \\
\hline 1 & 1 & 1 & 1 & 1 & -1 & -1 & 15 & -1 & 1 & 1 & 1 & 1 & -1 \\
2 & 1 & 1 & 1 & -1 & 1 & -1 & 16 & -1 & -1 & -1 & -1 & -1 & -1 \\
3 & 1 & 1 & -1 & 1 & -1 & 1 & 17 & $-\alpha$ & 0 & 0 & 0 & 0 & 0 \\
4 & 1 & -1 & 1 & -1 & 1 & 1 & 18 & $\alpha$ & 0 & 0 & 0 & 0 & 0 \\
5 & -1 & 1 & -1 & 1 & 1 & 1 & 19 & 0 & $-\alpha$ & 0 & 0 & 0 & 0 \\
6 & 1 & -1 & 1 & 1 & -1 & 1 & 20 & 0 & $\alpha$ & 0 & 0 & 0 & 0 \\
7 & -1 & 1 & 1 & -1 & -1 & -1 & 21 & 0 & 0 & $-\alpha$ & 0 & 0 & 0 \\
8 & 1 & 1 & -1 & -1 & 1 & 1 & 22 & 0 & 0 & $\alpha$ & 0 & 0 & 0 \\
9 & 1 & -1 & -1 & 1 & -1 & -1 & 23 & 0 & 0 & 0 & $-\alpha$ & 0 & 0 \\
10 & -1 & -1 & 1 & -1 & -1 & 1 & 24 & 0 & 0 & 0 & $\alpha$ & 0 & 0 \\
11 & -1 & 1 & -1 & -1 & -1 & 1 & 25 & 0 & 0 & 0 & 0 & $-\alpha$ & 0 \\
12 & 1 & -1 & -1 & -1 & 1 & -1 & 26 & 0 & 0 & 0 & 0 & $\alpha$ & 0 \\
13 & -1 & -1 & -1 & 1 & 1 & -1 & 27 & 0 & 0 & 0 & 0 & 0 & $-\alpha$ \\
14 & -1 & -1 & 1 & 1 & 1 & 1 & 28 & 0 & 0 & 0 & 0 & 0 & $\alpha$ \\
\hline
\end{tabular}


Tabela 9 - Delineamento composto quase saturado de DraperLin para sete fatores ( $\left.D L_{7}\right)$.

\begin{tabular}{lrrrrrrrrrrrrrrr}
\hline $\mathrm{N} 0$ & $\mathrm{X}_{1}$ & $\mathrm{X}_{2}$ & $\mathrm{X}_{3}$ & $\mathrm{X}_{4}$ & $\mathrm{X}_{5}$ & $\mathrm{X}_{6}$ & $\mathrm{X}_{7}$ & $\mathrm{~N} 0$ & $\mathrm{X}_{1}$ & $\mathrm{X}_{2}$ & $\mathrm{X}_{3}$ & $\mathrm{X}_{4}$ & $\mathrm{X}_{5}$ & $\mathrm{X}_{6}$ & $\mathrm{X}_{7}$ \\
\hline 1 & 1 & 1 & 1 & 1 & -1 & 1 & 1 & 20 & -1 & -1 & -1 & 1 & 1 & 1 & 1 \\
2 & 1 & 1 & 1 & -1 & 1 & -1 & 1 & 21 & -1 & -1 & -1 & 1 & 1 & 1 & -1 \\
3 & 1 & 1 & 1 & 1 & -1 & 1 & -1 & 22 & -1 & -1 & 1 & 1 & 1 & 1 & 1 \\
4 & 1 & 1 & -1 & -1 & 1 & 1 & -1 & 23 & -1 & 1 & 1 & 1 & 1 & -1 & -1 \\
5 & 1 & -1 & 1 & 1 & 1 & -1 & 1 & 24 & -1 & -1 & -1 & -1 & -1 & -1 & -1 \\
6 & -1 & 1 & -1 & 1 & -1 & -1 & 1 & 25 & $-\alpha$ & 0 & 0 & 0 & 0 & 0 & 0 \\
7 & 1 & -1 & 1 & -1 & -1 & 1 & -1 & 26 & $\alpha$ & 0 & 0 & 0 & 0 & 0 & 0 \\
8 & -1 & 1 & 1 & -1 & 1 & 1 & -1 & 27 & 0 & $-\alpha$ & 0 & 0 & 0 & 0 & 0 \\
9 & 1 & 1 & -1 & 1 & 1 & -1 & 1 & 28 & 0 & $\alpha$ & 0 & 0 & 0 & 0 & 0 \\
10 & 1 & -1 & -1 & 1 & -1 & -1 & -1 & 29 & 0 & 0 & $-\alpha$ & 0 & 0 & 0 & 0 \\
11 & -1 & -1 & 1 & -1 & -1 & 1 & 1 & 30 & 0 & 0 & $\alpha$ & 0 & 0 & 0 & 0 \\
12 & -1 & 1 & 1 & -1 & 1 & -1 & -1 & 31 & 0 & 0 & 0 & $-\alpha$ & 0 & 0 & 0 \\
13 & 1 & 1 & -1 & 1 & -1 & 1 & -1 & 32 & 0 & 0 & 0 & $\alpha$ & 0 & 0 & 0 \\
14 & 1 & -1 & -1 & -1 & 1 & -1 & -1 & 33 & 0 & 0 & 0 & 0 & $-\alpha$ & 0 & 0 \\
15 & -1 & -1 & 1 & 1 & -1 & -1 & -1 & 34 & 0 & 0 & 0 & 0 & $\alpha$ & 0 & 0 \\
16 & -1 & 1 & -1 & -1 & -1 & -1 & 1 & 35 & 0 & 0 & 0 & 0 & 0 & $-\alpha$ & 0 \\
17 & 1 & -1 & 1 & -1 & -1 & -1 & 1 & 36 & 0 & 0 & 0 & 0 & 0 & $\alpha$ & 0 \\
18 & -1 & 1 & -1 & -1 & -1 & 1 & 1 & 37 & 0 & 0 & 0 & 0 & 0 & 0 & $-\alpha$ \\
19 & 1 & -1 & -1 & -1 & 1 & 1 & 1 & 38 & 0 & 0 & 0 & 0 & 0 & 0 & $\alpha$ \\
\hline & & & & & & & & & & & & & &
\end{tabular}


Tabela 10 - Delineamento composto saturado de Draper-Lin para sete fatores $\left(D L_{7}\right)$.

№ \begin{tabular}{lllllllllllllll}
$\mathrm{x}_{1}$ & $\mathrm{x}_{2}$ & $\mathrm{x}_{3}$ & $\mathrm{x}_{4}$ & $\mathrm{x}_{5}$ & $\mathrm{x}_{6}$ & $\mathrm{x}_{7}$ & № & $\mathrm{x}_{1}$ & $\mathrm{x}_{2}$ & $\mathrm{x}_{3}$ & $\mathrm{x}_{4}$ & $\mathrm{x}_{5}$ & $\mathrm{x}_{6}$ & $\mathrm{x}_{7}$ \\
\hline
\end{tabular}

$\begin{array}{llllllllllllllll}1 & 1 & 1 & 1 & -1 & 1 & 1 & 1 & 19 & -1 & -1 & 1 & 1 & 1 & 1 & -1\end{array}$

$\begin{array}{llllllllllllllll}2 & 1 & 1 & -1 & 1 & -1 & 1 & -1 & 20 & -1 & -1 & 1 & 1 & 1 & -1 & 1\end{array}$

$\begin{array}{llllllllllllllll}3 & 1 & 1 & 1 & -1 & 1 & -1 & -1 & 21 & -1 & 1 & 1 & 1 & -1 & -1 & 1\end{array}$

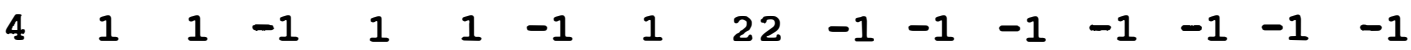

$\begin{array}{llllllllllllllll}5 & 1 & -1 & 1 & 1 & -1 & 1 & 1 & 23 & -\alpha & 0 & 0 & 0 & 0 & 0 & 0\end{array}$

$\begin{array}{llllllllllllllll}6 & -1 & 1 & 1 & -1 & -1 & 1 & -1 & 24 & \alpha & 0 & 0 & 0 & 0 & 0 & 0\end{array}$

$\begin{array}{llllllllllllllll}7 & 1 & -1 & -1 & -1 & 1 & -1 & -1 & 25 & 0 & -\alpha & 0 & 0 & 0 & 0 & 0\end{array}$

$\begin{array}{llllllllllllllll}8 & -1 & 1 & -1 & 1 & 1 & -1 & 1 & 26 & 0 & \alpha & 0 & 0 & 0 & 0 & 0\end{array}$

$\begin{array}{llllllllllllllll}9 & 1 & 1 & 1 & 1 & -1 & 1 & -1 & 27 & 0 & 0 & -\alpha & 0 & 0 & 0 & 0\end{array}$

$\begin{array}{llllllllllllllll}10 & 1 & -1 & 1 & -1 & -1 & -1 & 1 & 28 & 0 & 0 & \alpha & 0 & 0 & 0 & 0\end{array}$

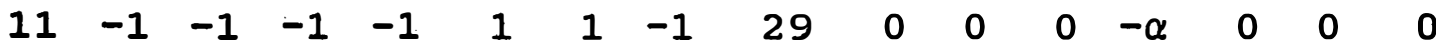

$\begin{array}{llllllllllllllll}12 & -1 & 1 & -1 & 1 & -1 & -1 & -1 & 30 & 0 & 0 & 0 & \alpha & 0 & 0 & 0\end{array}$

$\begin{array}{llllllllllllllll}13 & 1 & -1 & -1 & 1 & -1 & -1 & -1 & 31 & 0 & 0 & 0 & 0 & -\alpha & 0 & 0\end{array}$

$\begin{array}{llllllllllllllll}14 & -1 & -1 & 1 & -1 & -1 & -1 & 1 & 32 & 0 & 0 & 0 & 0 & \alpha & 0 & 0\end{array}$

$\begin{array}{llllllllllllllll}15 & -1 & 1 & -1 & -1 & -1 & 1 & 1 & 33 & 0 & 0 & 0 & 0 & 0 & -\alpha & 0\end{array}$

$\begin{array}{llllllllllllllll}16 & 1 & -1 & -1 & -1 & -1 & 1 & 1 & 34 & 0 & 0 & 0 & 0 & 0 & \alpha & 0\end{array}$

$\begin{array}{llllllllllllllll}17 & -1 & 1 & -1 & -1 & 1 & 1 & 1 & 35 & 0 & 0 & 0 & 0 & 0 & 0 & -\alpha\end{array}$

\begin{tabular}{llllllllllllllll}
18 & 1 & -1 & -1 & 1 & 1 & 1 & 1 & 36 & 0 & 0 & 0 & 0 & 0 & 0 & $\alpha$ \\
\hline
\end{tabular} 
Tabela 11 - Delineamento composto quase saturado de Westlake para cinco fatores $\left(W_{5}\right)$.

\begin{tabular}{lrrrrrrrrrrr}
\hline No & $\mathrm{X}_{1}$ & $\mathrm{X}_{2}$ & $\mathrm{X}_{3}$ & $\mathrm{X}_{4}$ & $\mathrm{X}_{5}$ & $\mathrm{~N} 0$ & $\mathrm{X}_{1}$ & $\mathrm{X}_{2}$ & $\mathrm{X}_{3}$ & $\mathrm{X}_{4}$ & $\mathrm{X}_{5}$ \\
\hline 1 & 1 & 1 & 1 & 1 & -1 & 12 & 1 & 1 & 1 & -1 & 1 \\
2 & 1 & 1 & -1 & -1 & -1 & 13 & $-\alpha$ & 0 & 0 & 0 & 0 \\
3 & -1 & 1 & 1 & -1 & -1 & 14 & $\alpha$ & 0 & 0 & 0 & 0 \\
4 & -1 & 1 & -1 & 1 & -1 & 15 & 0 & $-\alpha$ & 0 & 0 & 0 \\
5 & 1 & -1 & 1 & 1 & 1 & 16 & 0 & $\alpha$ & 0 & 0 & 0 \\
6 & 1 & -1 & -1 & -1 & 1 & 17 & 0 & 0 & $-\alpha$ & 0 & 0 \\
7 & -1 & -1 & 1 & -1 & 1 & 18 & 0 & 0 & $\alpha$ & 0 & 0 \\
8 & -1 & -1 & -1 & 1 & 1 & 19 & 0 & 0 & 0 & $-\alpha$ & 0 \\
9 & -1 & 1 & -1 & -1 & 1 & 20 & 0 & 0 & 0 & $\alpha$ & 0 \\
10 & -1 & 1 & 1 & 1 & 1 & 21 & 0 & 0 & 0 & 0 & $-\alpha$ \\
11 & 1 & 1 & -1 & 1 & 1 & 22 & 0 & 0 & 0 & 0 & $\alpha$ \\
\hline
\end{tabular}


Tabela 12 - Delineamento composto quase saturado de Westlake para sete fatores $\left(\mathrm{We}_{7}\right)$.

\begin{tabular}{lrrrrrrrrrrrrrrr}
\hline $\mathrm{N} 0$ & $\mathrm{X}_{1}$ & $\mathrm{X}_{2}$ & $\mathrm{X}_{3}$ & $\mathrm{X}_{4}$ & $\mathrm{X}_{5}$ & $\mathrm{X}_{6}$ & $\mathrm{X}_{7}$ & $\mathrm{~N} 0$ & $\mathrm{X}_{1}$ & $\mathrm{X}_{2}$ & $\mathrm{X}_{3}$ & $\mathrm{X}_{4}$ & $\mathrm{X}_{5}$ & $\mathrm{X}_{6}$ & $\mathrm{X}_{7}$ \\
\hline 1 & 1 & 1 & 1 & -1 & -1 & -1 & -1 & 21 & 1 & 1 & -1 & 1 & -1 & 1 & 1 \\
2 & 1 & -1 & 1 & 1 & -1 & -1 & -1 & 22 & -1 & -1 & 1 & -1 & 1 & 1 & 1 \\
3 & 1 & 1 & -1 & -1 & 1 & -1 & -1 & 23 & 1 & -1 & 1 & -1 & 1 & 1 & 1 \\
4 & 1 & -1 & -1 & 1 & 1 & -1 & -1 & 24 & -1 & 1 & 1 & 1 & 1 & 1 & 1 \\
5 & -1 & -1 & -1 & 1 & -1 & 1 & -1 & 25 & 1 & -1 & 1 & -1 & -1 & -1 & 1 \\
6 & 1 & 1 & -1 & 1 & -1 & 1 & -1 & 26 & 1 & 1 & -1 & 1 & 1 & -1 & 1 \\
7 & 1 & -1 & 1 & -1 & 1 & 1 & -1 & 27 & $-\alpha$ & 0 & 0 & 0 & 0 & 0 & 0 \\
8 & -1 & 1 & 1 & -1 & 1 & 1 & -1 & 28 & $\alpha$ & 0 & 0 & 0 & 0 & 0 & 0 \\
9 & -1 & 1 & -1 & -1 & -1 & -1 & 1 & 29 & 0 & $-\alpha$ & 0 & 0 & 0 & 0 & 0 \\
10 & 1 & 1 & -1 & -1 & -1 & -1 & 1 & 30 & 0 & $\alpha$ & 0 & 0 & 0 & 0 & 0 \\
11 & 1 & -1 & 1 & -1 & -1 & -1 & 1 & 31 & 0 & 0 & $-\alpha$ & 0 & 0 & 0 & 0 \\
12 & -1 & 1 & 1 & -1 & -1 & -1 & 1 & 32 & 0 & 0 & $\alpha$ & 0 & 0 & 0 & 0 \\
13 & -1 & -1 & -1 & 1 & -1 & -1 & 1 & 33 & 0 & 0 & 0 & $-\alpha$ & 0 & 0 & 0 \\
14 & -1 & 1 & 1 & -1 & 1 & -1 & 1 & 34 & 0 & 0 & 0 & $\alpha$ & 0 & 0 & 0 \\
15 & -1 & -1 & -1 & 1 & 1 & -1 & 1 & 35 & 0 & 0 & 0 & 0 & $-\alpha$ & 0 & 0 \\
16 & 1 & 1 & -1 & 1 & 1 & -1 & 1 & 36 & 0 & 0 & 0 & 0 & $\alpha$ & 0 & 0 \\
17 & -1 & -1 & 1 & 1 & 1 & -1 & 1 & 37 & 0 & 0 & 0 & 0 & 0 & $-\alpha$ & 0 \\
18 & 1 & -1 & 1 & 1 & 1 & -1 & 1 & 38 & 0 & 0 & 0 & 0 & 0 & $\alpha$ & 0 \\
19 & -1 & -1 & -1 & -1 & -1 & 1 & 1 & 39 & 0 & 0 & 0 & 0 & 0 & 0 & $-\alpha$ \\
20 & -1 & 1 & -1 & 1 & -1 & 1 & 1 & 40 & 0 & 0 & 0 & 0 & 0 & 0 & $\alpha$ \\
\hline & & & & & & & & & & & & & &
\end{tabular}


Tabela 13 - Delineamento composto saturado de Lucas para 4 fatores $\left(\mathrm{L}_{4}\right)$

\begin{tabular}{cccccccccc}
\hline No & $\mathrm{X}_{1}$ & $\mathrm{X}_{2}$ & $\mathrm{X}_{3}$ & $\mathrm{X}_{4}$ & $\mathrm{~N} 0$ & $\mathrm{X}_{1}$ & $\mathrm{X}_{2}$ & $\mathrm{X}_{3}$ & $\mathrm{X}_{4}$ \\
\hline 1 & 1 & 1 & 0 & 0 & 8 & $-\alpha$ & 0 & 0 & 0 \\
2 & 1 & 0 & 1 & 0 & 9 & $\alpha$ & 0 & 0 & 0 \\
3 & 1 & 0 & 0 & 1 & 10 & 0 & $-\alpha$ & 0 & 0 \\
4 & 0 & 1 & 1 & 0 & 11 & 0 & $\alpha$ & 0 & 0 \\
5 & 0 & 1 & 0 & 1 & 12 & 0 & 0 & $-\alpha$ & 0 \\
6 & 0 & 0 & 1 & 1 & 13 & 0 & 0 & $\alpha$ & 0 \\
7 & 0 & 0 & 0 & 0 & 14 & 0 & 0 & 0 & $-\alpha$ \\
& & & & & 15 & 0 & 0 & 0 & $\alpha$ \\
\hline
\end{tabular}


Tabela 14 - Delineamento composto saturado de Lucas para cinco fatores $\left(I_{5}\right)$.

\begin{tabular}{lccccccccccc}
\hline $\mathrm{N} N$ & $\mathrm{X}_{1}$ & $\mathrm{X}_{2}$ & $\mathrm{X}_{3}$ & $\mathrm{X}_{4}$ & $\mathrm{X}_{5}$ & $\mathrm{~N} \cong$ & $\mathrm{X}_{1}$ & $\mathrm{X}_{2}$ & $\mathrm{X}_{3}$ & $\mathrm{X}_{4}$ & $\mathrm{X}_{5}$ \\
\hline 1 & 1 & 1 & 0 & 0 & 0 & 12 & $-\alpha$ & 0 & 0 & 0 & 0 \\
2 & 1 & 0 & 1 & 0 & 0 & 13 & $\alpha$ & 0 & 0 & 0 & 0 \\
3 & 1 & 0 & 0 & 1 & 0 & 14 & 0 & $-\alpha$ & 0 & 0 & 0 \\
4 & 1 & 0 & 0 & 0 & 1 & 15 & 0 & $\alpha$ & 0 & 0 & 0 \\
5 & 0 & 1 & 1 & 0 & 0 & 16 & 0 & 0 & $-\alpha$ & 0 & 0 \\
6 & 0 & 1 & 0 & 0 & 0 & 17 & 0 & 0 & $\alpha$ & 0 & 0 \\
7 & 0 & 1 & 0 & 1 & 1 & 18 & 0 & 0 & 0 & $-\alpha$ & 0 \\
8 & 0 & 0 & 1 & 0 & 0 & 19 & 0 & 0 & 0 & $\alpha$ & 0 \\
9 & 0 & 0 & 1 & 1 & 1 & 20 & 0 & 0 & 0 & 0 & $-\alpha$ \\
10 & 0 & 0 & 0 & 1 & 1 & 21 & 0 & 0 & 0 & 0 & $\alpha$ \\
11 & 0 & 0 & 0 & 0 & 0 & & & & & & \\
\hline
\end{tabular}


Tabela 15 - Delineamento composto saturado de Lucas para seis fatores $\left(\mathrm{L}_{6}\right)$.

\begin{tabular}{cccccccccccccc}
\hline $\mathrm{N} 0$ & $\mathrm{X}_{1}$ & $\mathrm{X}_{2}$ & $\mathrm{X}_{3}$ & $\mathrm{X}_{4}$ & $\mathrm{X}_{5}$ & $\mathrm{X}_{6}$ & $\mathrm{~N} 0$ & $\mathrm{X}_{1}$ & $\mathrm{X}_{2}$ & $\mathrm{X}_{3}$ & $\mathrm{X}_{4}$ & $\mathrm{X}_{5}$ & $\mathrm{x}_{6}$ \\
\hline 1 & 1 & 1 & 0 & 0 & 0 & 0 & 15 & 0 & 0 & 0 & 0 & 1 & 1 \\
2 & 1 & 0 & 1 & 0 & 0 & 0 & 16 & 0 & 0 & 0 & 0 & 0 & 0 \\
3 & 1 & 0 & 0 & 1 & 0 & 0 & 17 & $-\alpha$ & 0 & 0 & 0 & 0 & 0 \\
4 & 1 & 0 & 0 & 0 & 1 & 0 & 18 & $\alpha$ & 0 & 0 & 0 & 0 & 0 \\
5 & 1 & 0 & 0 & 0 & 0 & 1 & 19 & 0 & $-\alpha$ & 0 & 0 & 0 & 0 \\
6 & 0 & 1 & 1 & 0 & 0 & 0 & 20 & 0 & $\alpha$ & 0 & 0 & 0 & 0 \\
7 & 0 & 1 & 0 & 1 & 0 & 0 & 21 & 0 & 0 & $-\alpha$ & 0 & 0 & 0 \\
8 & 0 & 1 & 0 & 0 & 1 & 0 & 22 & 0 & 0 & $\alpha$ & 0 & 0 & 0 \\
9 & 0 & 1 & 0 & 0 & 0 & 1 & 23 & 0 & 0 & 0 & $-\alpha$ & 0 & 0 \\
10 & 0 & 0 & 1 & 1 & 0 & 0 & 24 & 0 & 0 & 0 & $\alpha$ & 0 & 0 \\
11 & 0 & 0 & 1 & 0 & 1 & 0 & 25 & 0 & 0 & 0 & 0 & $-\alpha$ & 0 \\
12 & 0 & 0 & 1 & 0 & 0 & 1 & 26 & 0 & 0 & 0 & 0 & $\alpha$ & 0 \\
13 & 0 & 0 & 0 & 1 & 1 & 0 & 27 & 0 & 0 & 0 & 0 & 0 & $-\alpha$ \\
14 & 0 & 0 & 0 & 1 & 0 & 1 & 28 & 0 & 0 & 0 & 0 & 0 & $\alpha$ \\
\hline
\end{tabular}


Tabela 16 - Delineamento composto saturado de Lucas para sete fatores ( $\mathrm{L}_{7}$ ).

\begin{tabular}{rrrrrrrrrrrrrrrr}
\hline No & $\mathrm{X}_{1}$ & $\mathrm{X}_{2}$ & $\mathrm{X}_{3}$ & $\mathrm{X}_{4}$ & $\mathrm{X}_{5}$ & $\mathrm{X}_{6}$ & $\mathrm{X}_{7}$ & $\mathrm{~N} \cong$ & $\mathrm{X}_{1}$ & $\mathrm{X}_{2}$ & $\mathrm{X}_{3}$ & $\mathrm{X}_{4}$ & $\mathrm{X}_{5}$ & $\mathrm{X}_{6}$ & $\mathrm{X}_{7}$ \\
\hline 1 & 1 & 1 & 0 & 0 & 0 & 0 & 0 & 19 & 0 & 0 & 0 & 0 & 1 & 1 & 0
\end{tabular}

$\begin{array}{llllllllllllllll}2 & 1 & 0 & 1 & 0 & 0 & 0 & 0 & 20 & 0 & 0 & 0 & 0 & 1 & 0 & 1\end{array}$

$\begin{array}{llllllllllllllll}3 & 1 & 0 & 0 & 1 & 0 & 0 & 0 & 21 & 0 & 0 & 0 & 0 & 0 & 1 & 1\end{array}$

$\begin{array}{llllllllllllllll}4 & 1 & 0 & 0 & 0 & 1 & 0 & 0 & 22 & 0 & 0 & 0 & 0 & 0 & 0 & 0\end{array}$

$\begin{array}{llllllllllllllll}5 & 1 & 0 & 0 & 0 & 0 & 1 & 0 & 23 & -\alpha & 0 & 0 & 0 & 0 & 0 & 0\end{array}$

$\begin{array}{llllllllllllllll}6 & 1 & 0 & 0 & 0 & 0 & 0 & 1 & 24 & \alpha & 0 & 0 & 0 & 0 & 0 & 0\end{array}$

$\begin{array}{llllllllllllllll}7 & 0 & 1 & 1 & 0 & 0 & 0 & 0 & 25 & 0 & -\alpha & 0 & 0 & 0 & 0 & 0\end{array}$

$\begin{array}{llllllllllllllll}8 & 0 & 1 & 0 & 1 & 0 & 0 & 0 & 26 & 0 & \alpha & 0 & 0 & 0 & 0 & 0\end{array}$

$\begin{array}{llllllllllllllll}9 & 0 & 1 & 0 & 0 & 1 & 0 & 0 & 27 & 0 & 0 & -\alpha & 0 & 0 & 0 & 0\end{array}$

$\begin{array}{llllllllllllllll}10 & 0 & 1 & 0 & 0 & 0 & 1 & 0 & 28 & 0 & 0 & \alpha & 0 & 0 & 0 & 0\end{array}$

$\begin{array}{llllllllllllllll}11 & 0 & 1 & 0 & 0 & 0 & 0 & 1 & 29 & 0 & 0 & 0 & -\alpha & 0 & 0 & 0\end{array}$

$\begin{array}{llllllllllllllll}12 & 0 & 0 & 1 & 1 & 0 & 0 & 0 & 30 & 0 & 0 & 0 & \alpha & 0 & 0 & 0\end{array}$

$\begin{array}{llllllllllllllll}13 & 0 & 0 & 1 & 0 & 1 & 0 & 0 & 31 & 0 & 0 & 0 & 0 & -\alpha & 0 & 0\end{array}$

$\begin{array}{llllllllllllllll}14 & 0 & 0 & 1 & 0 & 0 & 1 & 0 & 32 & 0 & 0 & 0 & 0 & \alpha & 0 & 0\end{array}$

$\begin{array}{llllllllllllllll}15 & 0 & 0 & 1 & 0 & 0 & 0 & 1 & 33 & 0 & 0 & 0 & 0 & 0 & -\alpha & 0\end{array}$

$\begin{array}{llllllllllllllll}16 & 0 & 0 & 0 & 1 & 1 & 0 & 0 & 34 & 0 & 0 & 0 & 0 & 0 & \alpha & 0\end{array}$

$\begin{array}{llllllllllllllll}17 & 0 & 0 & 0 & 1 & 0 & 1 & 0 & 35 & 0 & 0 & 0 & 0 & 0 & 0 & -\alpha\end{array}$

\begin{tabular}{llllllllllllllll}
18 & 0 & 0 & 0 & 1 & 0 & 0 & 1 & 36 & 0 & 0 & 0 & 0 & 0 & 0 & $\alpha$ \\
\hline
\end{tabular}

